九州大学学術情報リポジトリ

Kyushu University Institutional Repository

Structure and Function of Snake Venom Metalloproteinase Family

武谷，浩之

https://doi.org/10.11501/3063849

出版情報：九州大学，1992，博士（理学），論文博士 バージョン：

権利関係 : 


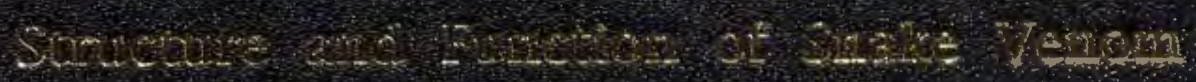

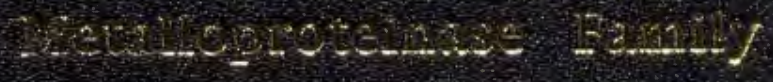

㱐酒 


\section{Structure and Function of Snake Venom Metalloproteinase Family}

\section{Hiroyuki Takeya}

Department of Biology, Faculty of Science, Kyushu University, Japan 


\section{CONTENTS}

\section{ABBREVIATIONS}

PREFACE

PART I : The High Molecular Mass Hemorrhagic Protein, HR1B, Isolated from the Venom of Trimeresurus flavoviridis

PART II : Coagulation Factor X Activating Enzyme from Russell's Viper Venom (RVV-X) : A NOVEL METALLOPROTEINASE WITH DISINTEGRIN (PLATELET AGGREGATION INHIBITOR) -LIKE AND C-TYPE LECTINLIKE DOMAIN 


\section{ABBREVIATIONS}

HR2a, hemorrhagic principle 2a;

Ht-d, hemorrhagic toxin d;

T., Trimeresurus;

C., Crotalus;

GPIIb/IIIa, platelet glycoprotein IIb/IIIa;

SDS-PAGE, sodium dodecyl sulfate-polyacrylamide gel electrophoresis; HPLC, high performance liquid chromatography;

CNBr, cyanogen bromide;

PTH, phenylthiohydantoin;

$\mathrm{RVV}-\mathrm{X}$, the factor $\mathrm{X}$ activating enzyme from Russell's viper venom; $R V V-V$, the factor $V$ activating enzyme from Russell's viper venom; Gla, $\gamma$-carboxyglutamic acid;

LC1, light chain 1;

IX/X-bp, factor IX/factor X-binding protein. 
PREFACE

Most snake venoms can be classified into several fundamental groups according to the main pathophysiological effects they manifest. Venoms of snakes belonging to families Elapidae (cobras, kraits, mambas, etc.) and Hydrophiidae (sea snakes) are highly neurotoxic and produce flaccid paralysis and respiratory failure in animals. Neurotoxins from these snake venoms have been extensively studied by many investigators. In contrast, Viperidae (viper) and Crotalidae (pit viper) venoms produce striking local effects, consisting of hemorrhage, necrosis, and edema, and often induce marked alterations of blood coagulation system as well. Among these pathological effects, hemorrhage is a most common occurrence in a victim bitten by crotalid and viperid snakes and various components, such as hemorrhagic factors and metalloproteinases, involved in these venoms have been isolated and characterized. These factors cause localized hemorrhage by direct actions on the blood vessel wall. Electron microscopic studies indicate that erythrocytes are leaked in a one-by-one fashion through widened inter-endothelial gaps when capillaries are exposed to these hemorrhagic proteins. The enzymes may disrupt the pericellular basement membrane through a proteolytic activity and with subsequent damage to the integrity of the vessel wall after which hemorrhage occurs. On the other hand, crotalid and viperid venoms contain many non-hemorrhagic metalloproteinases which act as procoagulants having very strict substrate specificities such as Russell's viper venom factor $\mathrm{X}$ activator (RVV-X).

In the 1970 era, four hemorrhagic metalloproteinases HR1A, $\mathrm{HR} 1 \mathrm{~B}, \mathrm{HR} 2 \mathrm{a}$, and HR2b, and one non-hemorrhagic $\mathrm{H}_{2}$-proteinase have been purified from the venom of Trimeresurus (T) flavoviridis. In the 
earlier reports, we established the primary structures of HR2a (202 residues) and $\mathrm{H}_{2}$-proteinase (201 residues), and other hemorrhagic metalloproteinase HT-2 (202 residues), isolated from the venom of Crotalus ruber ruber. All of these enzymes are very similar in sequence to each other and contain the putative active site sequence His-Glu-X-X-His that is homologous to the active sites of thermolysin and several other related bacterial enzymes as well as mammalian zinc metalloproteinases. However, since no significant sequence similarity beyond this region is found with any other known metalloproteinases, the snake venom enzymes belong to newly identified metalloproteinase subfamily. Among the venom metalloproteinases, the high molecular mass (Mr 60,000) hemorrhagic protein HR1B expresses 10 times higher hemorrhagic activities than does HR2a, thereby indicating major lethal factors in T. flavoviridis venom. In addition, HR1 (mixture of HR1A and HR1B) as well as the crude venom inhibit ADP-stimulated platelet aggregation.

In Part I of this thesis, the amino acid sequence of HR1B has been determined to explain these characteristic features of HR1B. In Part II, the entire amino acid sequence of RVV-X has been determined in order to elucidate the molecular mechanism of the RVV-X-catalyzed factor $\mathrm{X}$ activation, in particular how RVV-X specifically recognizes factor $\mathrm{X}$. 
PART I

The High Molecular Mass Hemorrhagic Protein, HR1B, Isolated from the Venom of Trimeresurus 'flavoviridis 


\section{SUMMARY}

Hemorrhage is a common occurrence in a victim bitten by crotalid and viperid snakes, and hemorrhagic components in these various venoms have been isolated and characterized. Previously, we have shown that a low molecular weight hemorrhagic protein (HR2a, 202 amino acid residues) isolated from the venom of Trimeresurus flavoviridis is a member of new subfamily of metalloproteinases. We now report the complete amino acid sequence of a high molecular mass hemorrhagic protein isolated from the same venom. This protein, HR1B, is a mosaic protein composed of 416 residues containing 4 Asnlinked oligosaccharide chains. The amino-terminal half (residues 1203) of HR1B contains a metalloproteinase domain, the sequence of which is 62 \& identical to that of HR2a and 52 \& identical to that of Ht-d isolated from the Crotalus atrox venom. The most interesting finding is that the middle region (residues 204-300) of HR1B shows a striking similarity to disintegrins, Arg-Gly-Asp-containing platelet aggregation inhibitors, recently found in several viper venoms. Interestingly, however, this region of HR1B does not contain the ArgGly-Asp-sequence which is known to be a putative binding site in the disintegrins for the platelet fibrinogen receptor, glycoprotein IIb/IIIa complex. We also found that the carboxy-terminal region (residues 213-336) of the middle part of HR1B shows $30 \%$ identity to residues 1543-1656 of von willebrand factor, and that the remaining region at the carboxyl-terminal end is unique and has a cysteine-rich sequence. These results suggest that the middle portion of HR1B, which shows structural similarities to the disintegrins and von Willebrand factor, may be important in synergistically stimulating hemorrhagic activity in the $\mathrm{NH}_{2}$-terminal metalloproteinase domain. 


\section{INTRODUCTION}

The neurotoxic effects of cobra and sea snake venoms are now well understood and purified neurotoxins serve as tools for the investigation of mammalian neuronal systems (1). In contrast, the local effects of crotalid and viperid venoms, toxins which cause severe hemorrhage, necrosis and edema, are poorly understood at the molecular level. Among factors responsible for these effects, hemorrhagic proteins have been highly purified and characterized (2). These proteins cause localized hemorrhage by direct actions on the blood vessel wall. Electron microscopic studies have shown that erythrocytes are broken in a one-by-one fashion through widened inter-endothelial gaps when capillaries are exposed to these hemorrhagic proteins $(3,4)$. Moreover, hemorragic proteins degrade basement membrane preparations (5) as well as isolated components, including type IV collagen, laminin, nidogen, and fibronectin (6). These findings suggest that hemorrhagic proteins may disrupt the pericellular basement membrane through proteolytic activity and with subsequent damage to the integrity of the vessel wall after which hemorrhage occurs $(6,7)$. The purified venom proteins should provide an appropriate tool for elucidating mechanisms related to the oozing of erythrocytes and plasma proteins from microcirculatory systems.

In 1989 Shannon et al. (6) and our group (7) reported the primary structures of low molecular weight hemorrhagic proteins (Mr 23,000), named hemorrhagic principle 2a (HR2a) and hemorrhagic toxin $\mathrm{d}(\mathrm{Ht}-\mathrm{d})$, isolated from the venom of Trimeresurus flavoviridis and Crotalus atrox, respectively. The structures revealed that both are members of a newly identified subfamily of 
metalloproteinases which have peptide segments similar to part of the zinc-chelating site and one of the catalytic residues of thermolysin. However, there is no significant sequence similarity to thermolysin or any other known metalloproteinases, except for the zinc chelating sequence.

High molecular weight (Mr 60,000-90,000) hemorrhagic proteins have also been isolated from the venom of various species of snakes including Agkistrodon halys blomhoffii $(8,9)$, C. atrox (10), and $C$. horridus horridus (11), all of which have potent hemorrhagic activity. Two high molecular weight hemorrhagic proteins, named HR1A and HR1B and isolated from the venom of $T$. flavoviridis $(12,13)$, are closely related, if not identical, immunologically. They have molecular weights of 60,000. HR1A and HR1B give LD50 values of 7.2 and $4.9 \mathrm{mg} /$ mouse, respectively, and show 10 times higher hemorrhagic activities than does HR2a, thereby indicating that they are major lethal factors in $T$. flavoviridis venom (13). In addition to these pathological functions, HR1 (a mixture of HRIA and HR1B) as well as the crude venom inhibit ADP-stimulated platelet aggregation (14). HR1 has the potential to inhibit $60 \%$ of platelet aggregation at a concentration of $6 \mathrm{ng} / \mathrm{ml}$, in platelet rich plasma. In situ microscopic observations also reveal that hemorrhagic proteins present in venom induce hemorrhage with little or no formation of white thrombi at the site of the hemorrhage $(3,4)$.

In ongoing work to elucidate the structure and function relationships of hemorrhagic components, we determined the complete amino acid sequence of $\mathrm{HR} 1 \mathrm{~B}$ and compared the findings with the low molecular mass hemorrhagic protein, HR2a. We obtained evidence that HR1B is a mosaic protein consisting of an 
amino-terminal metalloproteinase domain, a large noncatalytic middle segment with a trigramin-like structure (15-17) and a unique carboxyl-terminal Cys-rich domain. This is apparently the first report of the entire sequence of a high molecular mass hemorrhagic protein detected in crotalid and viperid venoms. 


\section{MATERIALS AND METHODS}

Materials The sources of materials used were as follows: Achromobacter lyticus lysyl endopeptidase, 4vinylpyridine and succinic anhydride from wako Pure Chemical Industries, Osaka; endoproteinase Asp-N from Boehringer-Mannheim Biochemica, FRG; L-pyroglutamylpeptidase from Suntory, Osaka; trypsin treated with $N$-tosyl-phenylalanyl chloromethyl ketone and a-chymotrypsin from Worthington Biochemical, Freehold, N. J.; arginylendopeptidase from Takara Shuzo Co. Ltd., Kyoto; cyanogen bromide, o-iodosobenzoic acid and Cosmosil (3C18, 5C4) columns from Nacalai Tesque, Inc., Kyoto; TSK ODS-120T and Phenyl-5pw RP columns from Toyo Soda Manufacturing Co. Ltd., Osaka; a Vydac 214 TP5415 column from The Separations Group, CA; reagents for gas-phase sequencer from Applied Biosystems, CA. All other chemicals were of analytical grade or of the highest quality commercially available.

Purification of HRIB HR1B was highly purified from

T. flavoviridis venom, as described (13). Briefly, the purification method consisted of three steps, gel filtration on Sephadex G-100, ion-exchange chromatography on DEAE-Sephadex A50, and gel filtration on Sephadex G-200 superfine. The purified preparation gave a single band on SDS-PAGE under reduced conditions and the apparent molecular mass was estimated to be approximately $\mathrm{Mr} 60,000$ (data not shown).

S-Pyridylethylation of HR1B HR1B (200 nmol) was incubated in $6 \mathrm{ml}$ of $6 \mathrm{M}$ guanidine hydrochloride containing $0.5 \mathrm{M}$ Tris-HCl, $10 \mathrm{mM}$ EDTA, and $40 \mathrm{mM}$ dithiothreitol, $\mathrm{pH} 8.6$, under nitrogen for $3 \mathrm{~h}$ at $50^{\circ} \mathrm{C}$. 4-vinylpyridine ( $1.2 \mathrm{nmol}$ ) was then 
added and the mixture was further incubated in the dark for $2.5 \mathrm{~h}$ at room temperature. The excess reagents were removed by dialysis against distilled water.

CNBr Digestion Pe-HR1B (100 nmol) in $2 \mathrm{ml}$ of $70 \%$ formic acid was treated with $\mathrm{CNBr}$, a 100-fold molar excess over methionine residues under nitrogen, and incubated at room temperature for $20 \mathrm{~h}$ in the dark. The reaction was terminated by lyophilization.

Subdigestion with Lysyl Endopeptidase CNBr fragments, M1, M5, and M6 (10 nmol each) were subdigested with lysyl endopeptidase. The peptides in $50 \mathrm{mM}$ Tris-HCl buffer, $\mathrm{pH}$ 9.0, containing $2 \mathrm{M}$ urea were digested with an enzyme/substrate ratio of $1 / 50(\mathrm{~mol} / \mathrm{mol})$ at $37{ }^{\circ} \mathrm{C}$ for $18 \mathrm{~h}$.

Subdigestion with Endoproteinase Asp-N- $=\mathrm{CNBr}$ fragments, M1, M5, and M6 ( 6 nmol each) were subdigested with endoproteinase Asp-N. The peptides in $50 \mathrm{mM}$ Tris-HCl buffer, $\mathrm{pH}$ 8.0, containing $1 \mathrm{M}$ urea were digested with an enzyme/substrate ratio of $1 / 150(\mathrm{~mol} / \mathrm{mol})$ at $37{ }^{\circ} \mathrm{C}$ for $18 \mathrm{~h}$.

Fragmentation with o-Iodosobenzoic Acid - $\mathrm{CNBr}$ fragment M1 was cleaved at tryptophanyl peptide bonds. The peptide $(6.5 \mathrm{nmol})$ was incubated in $0.2 \mathrm{ml}$ of $80 \%$ acetic acid containing $4 \mathrm{M}$ guanidine hydrochloride and $4.5 \mathrm{mM}$ o-iodosobenzoic acid for $24 \mathrm{~h}$ at room temperature, in the dark (18). Tryptic Digestion - Pe-HR1B was succinylated to protect the cleavage of lysyl peptide bonds before tryptic digestion. The Pe-protein (50 nmol) in $3 \mathrm{ml}$ of $0.1 \mathrm{M} \mathrm{NaHCO}, \mathrm{pH}$ 8.3, containing $8 \mathrm{M}$ urea was treated with succinic anhydride, a 3-fold molar excess over lysine residues. After addition of succinic anhydride, $\mathrm{pH}$ of the mixture dropped; it was adjusted to 
by adding $\mathrm{NaOH}$. The solution was allowed to stand for $1.5 \mathrm{~h}$ at room temperature. The O-succinyl groups were removed by $1 \mathrm{M}$ hydroxylamine, $\mathrm{pH} 8.5$, for $3 \mathrm{~h}$. The succinylated pe-HR1B in 0.75 $\mathrm{ml}$ of $50 \mathrm{mM}$ Tris-HCl buffer, $\mathrm{pH} 7.5$, containing $4 \mathrm{M}$ urea was digested by trypsin with an enzyme/substrate ratio of $1 / 150$ (mol/mol) at $37{ }^{\circ} \mathrm{C}$ for $23 \mathrm{~h}$.

Digestion with Arginylendopeptidase $\longrightarrow$ Pe-HR1B (50 $\mathrm{nmol}$ ) in $0.9 \mathrm{ml}$ of $50 \mathrm{mM}$ Tris-HCl buffer, $\mathrm{pH} 8.0$, containing $2 \mathrm{M}$ urea was digested with an enzyme/substrate ratio of $1 / 150$ (mol/mol) at $37{ }^{\circ} \mathrm{C}$ for $6 \mathrm{~h}$.

Peptide Purification P Peptides were purified by reversed-phase HPLC in 0.1 \& trifluoroacetic acid with acetonitrile gradient elution at a flow rate of $0.5 \mathrm{ml}$ per min. Details are the same as described (19).

Amino Acid Analysis and Sequence Determination The amino acid analysis of the pe-protein was performed by ionexchange chromatography in a Hitachi model L-8500 high speed amino acid analyzer after hydrolysis with $5.7 \mathrm{M} \mathrm{HCl}$ containing $0.2 \%$ phenol at $110^{\circ} \mathrm{C}$ for 24,48 , and $72 \mathrm{~h}$ by the method of Spackman et al. (20). Tryptophan was determined by hydrolysis in $3 \mathrm{~N}$ mercaptoethanesulfonic acid (21). The CNBr fragments and arginylendopeptidase fragments were analyzed using a Hitachi L8500 amino acid analyzer after hydrolysis with $5.7 \mathrm{M} \mathrm{HCl}$ containing 0.28 phenol at $110^{\circ} \mathrm{C}$ for $24 \mathrm{~h}$. The tryptic peptides and subdigested peptides were analyzed using reversed-phase HPLC of phenylthiocarbamoyl derivatives (22), using a Waters PICO-TAG system, after hydrolysis with $5.7 \mathrm{M} \mathrm{HCl}$ containing $1 \&$ phenol at $110{ }^{\circ} \mathrm{C}$ for $20 \mathrm{~h}$. Automated sequence analyses were performed with an Applied Biosystems 477A protein sequencer, as described by 
Hewick et al (23), with an Applied Biosystems model 120A PTH analyzer.

Determination of the Carboxyl-Terminal Amino Acid The carboxyl-terminal amino acid of HR1B was determined by the vapor-phase hydrazinolysis method (24). Highly dried 10 nmol of protein was treated with vaporized hydrazine at $90{ }^{\circ} \mathrm{C}$ for $3 \mathrm{~h}$. The hydrazinolysate was then treated with benzaldehyde. The amino acid released from the carboxyl-terminus of the protein was identified with a Hitachi model L-8500 amino acid analyzer.

Nomenclature of the Peptides Peptides are designated by a serial number prefixed by a letter. The letters indicated the type of digestion: M, CNBr; K, lysyl endopeptidase; D, endoproteinase Asp $-\mathrm{N} ; \mathrm{R}$, arginylendopeptidase; ST, tryptic digestion of succinylated protein; W, o-iodosobenzoic acid. The numbers in the peptide designation do not correspond to the order of their elution in HPLC, but rather to their positions in the protein sequence, starting from the $\mathrm{N}$-terminus. 


\section{RESULTS}

Amino Acid Composition and Sequence Analysis of HR1B - The amino acid composition of HRIB determined from the sequence as well as that obtained by amino acid analysis is shown in Table I. Based on the analysis, HR1B contained glucosamine but not galactosamine. HR1B had a high content of cysteine. Sequence analyses of intact protein ( $1 \mathrm{nmol})$ and Pe-protein (2 nmol) revealed no PTH derivatives up to 5-cycles of Edman degradation, thereby indicating that the amino-terminus is blocked, as are those of HR2a (7) and Ht-d (6). By treatment of intact HR1B with pyroglutamylpeptidase, the amino-terminal pyroglutamyl residue was released to give HR1B PG and the resultant protein HR1B PG was sequenced (Fig. 1). As with findings with HR2a (7) and Ht-d (6), sequence analysis of HR1B PG revealed the presence of a variant of the sequence lacking glutamine at position 2. Vapor-phase hydrazinolysis of HR1B yielded $0.11 \mathrm{~mol}$ of alanine, $0.07 \mathrm{~mol}$ of lysine and $0.13 \mathrm{~mol}$ of serine per mol of protein (uncorrected), hence, there is probably microheterogenity at the carboxylterminus of HR1B.

CNBr Peptides The entire amino acid sequence of HR1B was determined primarily from a set of methionyl-cleavage fragments (Fig. 1). The CNBr digest of pe-HR1B was separated by reversed-phase HPLC on a column of Vydac 214 TP5415 (Fig. 2). Peptides M4-5 and M5 were separated by rechromatography on a column of ODS-120T (Fig. 3). The minor peptide M7" was separated from M7' by rechromatography as shown in Fig. 4. The amino acid compositions and sequences of obtained peptides are shown in Tables II and III, and Fig. 1. M5-DP was a peptide which was 
partially hydrolyzed at Asp-Pro bond in 70 \& formic acid during digestion. M4-5 and M3-4-5 were yielded by incomplete cleavage at a Met-Ile bond (residues 170-171), and a Met-Ser bond (residues167-168). Similar but not identical peptides, M7, M7' and M7" did not contain homoserine in their compositions, thereby indicating that these peptides originated from the carboxylterminal end. Amino acid sequences of M7, M7' and M7" were complete, showing that M7' contained an extended sequence of TyrLys and M7" had a more extended sequence of Tyr-Lys-Ser from the carboxyl-terminus of M7. As described above, the result obtained on hydrazinolysis of intact protein indicated three carboxylterminal residues, Ala, Lys and Ser. Thus, the carboxyl-terminal ragged ends of HR1B, such as, -Ala, -Ala-Tyr-Lys and -Ala-Tyr-LysSer, were confirmed. The amino acid sequences of CNBr peptides, M1, M5 and M6 were determined completely from two overlapping sets of peptides generated on digestion with lysyl endopeptidase and endoproteinase Asp-N.

Subdigestion of M1- Subdigests of M1 with lysyl endopeptidase yielded 8 peptides (Fig. 5). The amino acid compositions and sequences of the peptides are shown in Tables III and IV, and Fig. 1. The amino acid composition and blocked aminoterminus indicated that M1K1 originated from the amino-terminal end. After digestion with pyroglutamylpeptidase, the resultant peptide M1KI PG could be sequenced (Tables III and IV, and Fig. 1). Although the entire HRIB had the amino-terminal ragged end, sequence analysis of M1K1 PG did not reveal the presence of a variant peptide with glutamine at position 2 . One possible explanation is that the glutamine residue newly appearing at the amino-terminal end of this variant peptide by the 
pyroglutamylpeptidase treatment was cyclized again and this newly cyclized glutamine was further digested by pyroglutamylpeptidase and could be sequenced only from the arginine at position 3. M1K6 and M1K6' gave the same amino acid compositions (Table IV). However, M1K6' could not be sequenced, suggesting that cyclization of the amino-terminal glutamine of M1K6 might have occurred during digestion or HPLC. Subdigests of M1 with endoproteinase Asp-N yielded 6 peptides (Fig. 6). Amino acid compositions of these six peptides are shown in Table V. As shown in Table III and Fig. 1, M1D2 overlapped M1K2, M1K3, M1K4, and M1K5. M1D4 and M1D6 overlapped M1K5, M1K6 and M1K7. M1W1, generated by cleavage at tryptophanyl residues, confirmed the carboxyl-terminal sequence of M1D2. Hence, a continuous sequence of M1 was obtained. Subdigestion of M5 Subdigests of M5 with lysyl endopeptidase were separated by reversed-phase HPLC (Figs. 7 and 8). The total of 6 peptides were subjected to amino acid and sequence analyses (Tables III and VI, and Fig. 1). Twelve peptides were obtained from subdigests of M5 with endoproteinase Asp-N (Figs. 9-11), and their amino acid and sequence data are shown in Tables III and VII and Fig. 1. M5D7 overlapped M5K3, M5K4 and M5K5. M5D12 filled the carboxyl-terminal portion of M5K5 .

$$
\text { Subdigestion of M6 Two subdigestions of M6 were }
$$
performed by cleavage with lysyl endopeptidase and endoproteinase Asp-N. The resulting fragments were separated by reversed-phase HPLC (Figs. 12-15). Peptides obtained were subjected to structural analyses (Tables III, VIII, and IX and Fig. 1). M6K4 and M6K5 overlapped by M6D6 provided the remainder of the sequence of $\mathrm{M} 6$. 
Cleavage at Arginyl Residues Alignment of the

methionyl-cleavage fragments was obtained by analyses of the peptides isolated from cleavages at the arginyl residues. Pe-HR1B was cleaved by trypsin at arginyl residues after $N$-succinylation. The peptides were separated by reversed-phase HPLC as shown in Fig. 16. The amino acid compositions of the peptides are shown in Table $\mathrm{X}$. The peak just before ST1 seemed to be an uncleaved peptide of ST1 and ST2, as determined by the amino acid composition (data not shown) and the blocked amino terminus. The amino acid sequence of ST2 overlapped M1 and M2 (Table III, and Fig. 1). The sequences of ST4 and ST4-5 overlapped M5K5 and M5D12. The sequence of ST5 overlapped M5D12, M5K6 and M6. Since ST6 overlapped M6K5 and M7, M6 and M7 were linked. The overlap between M2 and M3 was achieved by the sequence analysis of R1, one of the fragments of Pe-HR1B digested with arginylendopeptidase (Fig. 17 and Table XI).

Carbohydrate-Linked Asparagine Residues - The PTH derivatives at positions $73,181,327$ and 380, were not identified by sequence analysis. These are very likely carbohydrate-linked Asn residues since they are followed by a -X-Thr/Ser sequence, a consensus signal sequence for the attachment of carbohydrate to asparagine and because the composition analyses of the fragments containing these residues show the presence of glucosamines in each fragment. The reasonably high yields for Thr and ser residues in the entire sequence and composition analyses of all the fragments, including intact protein, indicate the absence of O-linked carbohydrate chains in HR1B.

Summary of Sequence Analyses of HR1B — Ninety-seven percent of the total sequence was determined by two or three 
independent analyses using different peptides, as shown in Fig. 1. HR1B was composed of 416 residues with 4 Asn-linked sugar chains at positions $73,181,327$ and 380 . HR1B has been reported to contain neutral sugar, amino sugar and sialic acid accounting for 17-18 $\%$ on a total weight basis (13) and corresponding to 10-11 kilodaltons. The molecular weight for the polypeptide portion of the largest isoform was calculated to be 46,478 , thus the molecular weight was approximately 57,000 with the addition of the four carbohydrate chains. This number differed slightly from the reported Mr of 60,000 (13), as estimated by SDS-PAGE. In all likelihood, this difference was due to abnormal migration of glycoproteins on SDS-PAGE (25). The net charge of the polypeptide portion of HR1B was calculated to be +6 . Since the isoelectric point of HR1B was determined to be 4.4 (13), the sialic acid residues of the complex carbohydrate moieties must account for the acidic isoelectric point.

Titration of the Free SH-Group Since structural analyses of HR1B gave a total of 35 cysteine residues, as indicated in Table 1 and Fig. 1, the content of the free SH-group was determined by the method of Ellman (26) and incorporation of 4-vinylpyridine in the absence and presence of a denaturant. The values determined by the two methods with Ellman's reagent $\left(5,5^{\prime}-\right.$ dithiobis (2-nitrobenzoic acid)) and with s-pyridylethylation were in good agreement, showing that about $1 \mathrm{~mol}$ of $\mathrm{SH}$-group per mol of HR1B was titrated only in the presence of denaturant (Table XII). HR1B probably has a free cysteine residue present in the molecule, which is in striking contrast to the case of a low molecular mass of the hemorrhagic protein HR2a. 


\section{DISCUSSION}

We reported the complete amino acid sequence and the disulfide bridge locations of the hemorrhagic (HR2a) and nonhemorrhagic $\left(\mathrm{H}_{2}\right.$-proteinase) metalloproteinases $(7,27)$, isolated from snake venom used as the source of HR1B. In the same year, the amino acid sequence of Ht-d, a hemorrhagic metalloproteinase from the venom of $C$. atrox, was reported by other investigators (6). These are very similar metalloproteinases consisting of 201203 residues. As shown in Fig. 18A, the sequence of the aminoterminal half (203 residues) of HR1B represents a metalloproteinase structure similar to the low molecular weight metalloproteinases, HR2a, $\mathrm{H}_{2}$-proteinase and Ht-d. This metalloproteinase domain of HR1B contains an amino acid sequence (residues 143-147) consisting of the putative zinc-chelating (His143, 147) and catalytic (Glu-144) residues (Fig. 18A), identified by homology to the zinc-chelating sequence of thermolysin $(6,7$, 27). This domain of HR1B has a slightly higher level of identity to the sequences of HR2a $(62 \%)$ and $\mathrm{H}_{2}$-proteinase $(62 \%)$ than to that of Ht-d (52 \%), probably due to snake species differences. On the other hand, when the sequence identities of the three proteins from the same species are compared, HR2a and $\mathrm{H}_{2}-$ proteinase are more closely related ( $74 \%$ identity) than are HR2a/H2 proteinase and HR1B, thereby suggesting that HR1B diverged before the divergence of HR2a (hemorrhagic) and $\mathrm{H}_{2}$-proteinase (non-hemorrhagic).

The most characteristic feature found in the metalloproteinase domain of HR1B is the presence of two sugar chains (Fig. 18A). One linked to Asn-73 is in a rather 
hydrophobic region (residues 40-80) of HR1B, as predicted by the method of Kyte and Doolittle (28). Since the corresponding regions of the three other molecules, HR2a, $\mathrm{H}_{2}$-proteinase and Htd, are also hydrophobic, only HR1B is likely to have hydrophilicity in this region. Although $\mathrm{H}_{2}$-proteinase also has a potential carbohydrate binding sequence at Asn-73 (Fig. 18A), no carbohydrate moiety was detected (27). The other sugar chain located in HR1B is linked with Asn-181, at a position where Asp residues are followed by the $-\mathrm{X}$-Ser sequence in the three other molecules.

As we reported, $\mathrm{HR} 2 \mathrm{a}$ and $\mathrm{H}_{2}$-proteinase have similar primary structures although the latter does not have hemorrhagic activity (27). We compared the three hemorrhagic metalloproteinases (HR1B, HR2a and Ht-d) and one non-hemorrhagic $\mathrm{H}_{2}$-proteinase (Fig. 18A), to determin the structural element related to hemorrhaging. However, we found no clear differences between the hemorrhagic and non-hemorrhagic metalloproteinases. While there are few residues in common, at least six are shared by hemorrhagic but not by nonhemorrhagic proteinases (boxed residues in Fig. 18A). Such conserved residues may possibly be related to the induction of hemorrhagic activity. Further investigations are ongoing to evaluate the significance of these specific residues in relation to the substrate specificity of hemorrhagic proteinases.

Platelet aggregation inhibitory peptides, termed trigramin, echistatin, applaggin and bitistatin, have been isolated from the venom of T. gramineus, Echis carinatus, Agkistrodon piscivorus piscivorus and Bitis arietans, respectively, and the amino acid sequences have been determined $(15-17,29-32)$. These peptides are homologous cysteine rich peptides consisting of 49-83 residues. A 
highly conserved structural feature in these peptides is the tripeptide unit Arg-Gly-Asp (see Fig. 18B), a sequence that appears in a variety of adhesive protein ligands, such as fibronectin, vitronectin, fibrinogen, and von Willebrand factor, and contributes to their interaction with specific membrane receptors, called integrins $(33,34)$. These peptides, now known as the disintegrin family (35) are competitive inhibitors of fibrinogen and von Willebrand factor binding to the activated platelet membrane integrin, glycoprotein IIb/IIIa (GPIIb/IIIa) complex $(16,17)$. Trigramin is about 500 times more potent than a tetra- or hexapeptide containing Arg-Gly-Asp-sequence in inhibiting the binding of fibrinogen to the GPIIb/IIIa (16). It has also been reported that HRIB is a potent inhibitor of platelet aggregation (14). Here, we propose the structural elements for this activity based on the sequence similality. As shown in Fig. 18B, the cysteine-rich middle portion (residues 204-300) following the metalloproteinase domain of $\mathrm{HR} 1 \mathrm{~B}$ has a close similarity to the disintegrins. The sequence similarity of HR1B is scored $54 \%$ for trigramin, $34 \%$ for echistatin, $57 \%$ for applaggin, and $60 \%$ for bitistatin. Bitistatin most closely resembles the cysteine-rich middle portion of HRIB, in terms of not only the highest degree of sequence identity but also in other respects. Bitistatin is the largest disintegrin ( 83 residues) to have been isolated and the amino-terminal 10 residues unique to bitistatin are identical to the corresponding residues of HR1B, except for the conservative substitution of Leu to Ile. Two cysteine residues at positions 214 and 233 in HR1B are also present in bitistatin, but not in other disintegrins. It is noteworthy, however, that HR1B has one 
additional unique cysteine residue at position 276 not present in bitistatin.

The disintegrins bind to the GPIIb/IIIa complex through the Arg-Gly-Asp-sequence $(16,17)$. However, the Arg-Gly-Asp-sequence is absent in the HR1B molecule and is replaced by Glu-Ser-Glu-Cys (Fig. 18B). Several lines of evidence suggest that platelet aggregation inhibitory activity resides within the disintegrinlike domain of HR1B, despite the absence of the Arg-Gly-Aspsequence. First, it is suggested that a particular conformation of disintegrin is a prerequisite for its binding to the GPIIb/IIIa complex, since the chemically reduced sample does not inhibit fibrinogen and von Willebrand factor binding to activated platelets $(16,17,29)$. Second, in experiments using synthetic analogues of echistatin, it was noted that the Arg residue in the Arg-Gly-Asp-sequence plays a less important role in the binding of echistatin to activated platelets than does that of the Arg-GlyAsp-containing tetrapeptide (29). Finally, the tyrosylpentadecapeptide (Tyr-Gly-Gln-His-His-Leu-Gly-Gly-Ala-LysGln-Ala-Gly-Asp-Val) corresponding to the carboxyl-terminal sequence of the fibrinogen $\gamma$ chain blocks fibrinogen binding and trigramin binding to platelets (16), thereby suggesting that the Arg-Gly-Asp-sequence present in the snake venom disintegrin family is not the only sequence binding to platelets. It remains to be determined which structural segment of HR1B is essential for the inhibition of platelet aggregation. Synthetic fragments or fragments derived from native HR1B should prove useful in such cases. A peptide termed $\mathrm{CM}-2$ has been isolated from crude venom used for the source of bitistatin (36). CM-2 shows sequence identity to disintegrin and interestingly has the sequence of Arg- 
Gly-Asn in place of the Arg-Gly-Asp-sequence of disintegrin. Its platelet aggregation inhibitory activity has not yet been documented.

It is also of interest that the sequence of the disintegrinlike domain, including the amino-terminal part of the unknown cysteine-rich region in HR1B, shows a weak but statistically significant similarity to part of the sequence of human von willebrand factor (Fig. 19). Residues 213-336 of HR1B show $30 \%$ identity to residues 1543-1656 of von willebrand factor, for which the probability is less than $1.5 \times 10^{-6}$ that the sequence similarity arose by chance. The von willebrand factor participates in the initial reactions of hemostasis by mediating the adhesion of platelets to the subendothelium as well as the aggregation of platelets at the sites of vascular injury (37). Therefore, these structural similarities may be important in discerning the hemorrhagic function of HR1B, since both proteins have the same targets, e.g. platelets and the subendothelium.

Trigramin has been noted to inhibit the adhesion of melanoma cells to fibronectin (38). In this regard, the disintegin-like domain of HR1B may be important in localizing the HR1B molecule to the peripheral blood vessel, especially to integrins located in the basement membrane attachment site of the endothelial cells, when the metalloproteinase domain of HRIB degrades the basement membrane. This localization of HR1B to the subendothelium, in addition to its platelet aggregation inhibitory activity, may be one reason why HR1B expresses 10 times higher hemorrhagic activity than does HR2a (13).

The carboxyl-terminal region (residues 301-416) of HR1B contains numerous cysteine residues and two sugar chains (Fig. 
18B). This region is unique and not homologous to any known protein sequence. It seems likely that this region may also play an important role in inhibiting platelet aggregation and the cell adhesion inhibitory activities of HR1B, since the sequence GlnGlu-Asp-Val (positions 360-363) found in this region resembles the Arg-Glu-Asp-Val-sequence corresponding to the second cell attachment site in fibronectin (39).

The well known matrix-degrading metalloproteinases are members of the collagenase family. They have large noncatalytic hemopexin-like segments attached to the carboxyl-terminal ends of the metalloproteinase domains $(40-45)$, as noted with human interstitial collagenase (40), human stromelysin (41, 42), and rat stromelysin (transin (43)). Type IV collagenase (gelatinase) additionally contains a fibronectin-like collagen binding domain (45). However, in comparison with these mosaic proteinases, there is no significant structural similarlity to HR1B. Further experiments are required to determine whether the noncatalytic segment of HR1B is essential for the biological function of this enzyme, and/or for regulating proteolytic activity. 
1. Karlsson, E. (1979) in Handbook of Experimental Pharmacology Vol 52, Snake Venoms (Lee, C.-Y., ed) pp. 159-219, Springer-Verlag, Berlin, Heidelberg, New York

2. Tu, A.T. (1988) in Hemostasis and Animal Venoms (Pirkle, H., and Markland, F.S., Jr., eds) pp. 425-443, Marcel Dekker, New York

3. Tsuchiya, M., Ohshio, C., Ohashi, M., Ohsaka, A., Suzuki, K., and Fujishiro, Y. (1974) in Platelets, Thrombosis, and Inhibitors (Didisheim, P., ed) pp. 439-446, F. K. Schattauer Verlag, Stuttgart, New York

4. Ohsaka, A. (1979) in Handbook of Experimental Pharmacology (Lee, C.-Y., ed) Vol. 52, pp. 480-546, Springer-Verlag, Berlin

5. Ohsaka, A., Just, M., and Habermann, E. (1973) Biochim. Biophys. Acta $323,415-428$

6. Shannon, J.D., Baramova, E.N., Bjarnason, J.B., and Fox, J.W. (1989) J. Biol. Chem. 264, 11575-11583

7. Miyata, T., Takeya, H., Ozeki, Y., Arakawa, M., Tokunaga, F., Iwanaga, S., and Omori-Satoh, T. (1989) J. Biochem. (Tokyo) 105, $847-853$

8. Ohshima, G., Iwanaga, S., and Suzuki, T. (1968) J. Biochem. (Tokyo) 64, 215-225

9. Ohshima, G., Omori-Satoh, T., Iwanaga, S., and Suzuki, T. (1972) J. Biochem. (Tokyo) 72, 1483-1494

10. Bjarnason, J.B., and Tu, A.T. (1978) Biochemistry 17, 3395-3404 11. Civello, D.J., Duong, H.L., and Geren, C.R. (1983) Biochemistry 22, 749-755

12. Omori-Satoh, T., and Ohsaka, A. (1970) Biochim. Biophys. Acta $207,432-444$ 
13. Omori-Satoh, T., and Sadahiro, S. (1979) Biochim. Biophys. Acta $580,392-404$

14. Yamanaka, M., Matsuda, M., Isobe, J., Ohsaka, A., Takahasi, T., and Omori-Satoh, T. (1974) in Platelets, Thrombosis, and Inhibitors (Didisheim, P., ed) pp. 335-344, F. K. Schattauer Verlag, Stuttgart, New York

15. Ouyang, C., and Huang, T.-F. (1983) Biochim. Biophys. Acta 757, $332-341$

16. Huang, T.-F., Holt, J.C., Lukasiewicz, H., and Niewiarowski, S. (1987) J. Biol. Chem. 262, 16157-16163

17. Huang, T.-F., Holt, J.C., Kirby, E.P., and Niewiarowski, S. (1989) Biochemistry 28, 661-666

18. Mahoney, W.C., and Hermodson, M.A. (1979) Biochemistry 18, $3810-3814$

19. Takeya, H., Kawabata, S., Nakagawa, K., Yamamichi, Y., Miyata, T., Iwanaga, S., Takao, T., and Shimonishi, Y. (1988) J. Biol. Chem. 263, 14868-14877

20. Spackman, D.H., Stein, W.H., and Moore, S. (1958) Anal. Chem. $30,1190-1206$

21. Penke, B., Ferenczi, R., and Kovacs, K. (1974) Anal. Biochem. $60,45-50$

22. Bidlingmeyer, B.A., Cohen, S.A., and Tarvin, T.L. (1984) J. Chromatogr. $336,93-104$

23. Hewick. R.M., Hunkapiller, M.W., Hood, L.E., and Dreyer, W.J. (1981) J. Biol. Chem. 256, 7990-7997

24. Yamamoto, A., Toda, H., and Sakiyama, F. (1989) J. Biochem. (Tokyo) 106, 552-554

25. Segret, J.P. and Jackson, R.L. (1972) Methods Enzymol. 28, 54-63 26. Ellman, G.L. (1969) Arch. Biochem. Biophys. 82, 70-77 
27. Takeya, H., Arakawa, M., Miyata, T., Iwanaga, S., and OmoriSatoh, T. (1989) J. Biochem. (Tokyo) 106, 151-157

28. Kyte, J., and Doolittle, R.F. (1982) J. Mol. Biol. 157, 105-132

29. Gan, Z.-R., Gould, R.J., Jacobs, J.W., Friedman, P.A., and Polokoff, M.A. (1988) J. Biol. Chem. 263, 19827-19832

30. Garsky, V.M., Lumma, P.K., Ereidinger, R.M., Pitzenberger, S.M., Randall, W.C., Veber, D.F., Gould, R.J., and Friedman, P.A.

(1989) Proc. Nat1. Acad. Sci. USA 86, 4022-4026

31. Chao, B.H., Jakubowski, J.A., Savage, B., Chow, E.P., Marzec, U.M., Harker, L.A., and Maraganore. J.M. (1989) Proc. Natl.

Acad. Sci. USA 86, 8050-8054

32. Shebuski, R.J., Ramjit, D.R., Bencen, G.H., and Polokoff, M.A. (1989) J. Biol. Chem. 264, 21550-21556

33. Ruoslahti, E., and Pierschbacher, M.D. (1987) Science 238, 491497

34. Hynes, R.O. (1987) Cell 48, 549-554

35. Niewiarowski, S., Huang, T.-F., Rucinski, B., Cook, J.J., Williams, J.A., Musial, J., Edmunds, L.H., Jr., Gould, R.J., Bush, L., Shebuski, R., and Friedman, P.A. (1989) Thrombosis and Haemostasis 62,319

36. Joubert, F.J., Haylett, T., Strydom, D.J., and Taljaard, N. (1982) Hoppe-Seyler's Z. Physiol. Chem. 263, 1087-1096

37. Titani, K., Kumar, S., Takio, K., Ericsson, L.H., Wade, R.D., Ashida, K., Walsh, K.A., Chopek, M.W., Sadler, J.E., and Fujikawa, K. (1986) Biochemistry 25, 3171-3184

38. Knudsen, K.A., Tuszynski, G.P., Huang, T.-F., and Niewiarowski, S. (1988) Exp. Cel1 Res. 179, 42-49

39. Humphries, M.J., Akiyama, S.K., Komoriya, A., Olden, K., and Yamada, K.M. (1986) J. Cell Biol. 103, 2637-2647 
40. Goldberg, G.I., Wilhelm, S.M., Kronberger, A., Bauer, E.A., Grant, G.A., and Eisen, A.Z. (1986) J. Biol. Chem. 261, 66006605

41. Whitham, S.E., Murphy, G., Angel, P., Rahmsdorf, H.-J., Smith, B.J., Lyons,A., Harris, T.J.R., Reynolds, J.J., Herrlich, P., and Docherty, A.J.P. (1986) Biochem. J. 240, 913-916

42. Wilhelm, S.M., Collier, L.E., Kronberger, A., Eisen, A.Z. Marmer, B.L., Grant, G.A., Bauer, E.A., and Goldberg, G.I., (1987) Proc. Natl. ACad. SCi. USA 86, 6725-6729

43. Matrisian, L.M., Leroy, P., Ruhlmann, C., Gesnel, M.-C., and Breathnach, R. (1986) Mol. Cell. Biol. 6, 1679.-1686

44. Muller, D., Quantin, B., Gesnel, M.-C., Millon-Collard, R., Abecassis, J., and Breathnach, R. (1988) Biochem. J. 253, 187-192 45. Collier, I.E., Wilhelm, S.M., Eisen, A.Z. Marmer, B.L., Grant, G.A., Seltzer, J.L., Kronberger, A., He, C., Bauer, E.A., and Goldberg, G.I., (1988) J. Biol. Chem. 263, 6579-6587 
Table I

Amino acid composition of HRIB

Amino acid Analysis a Sequence

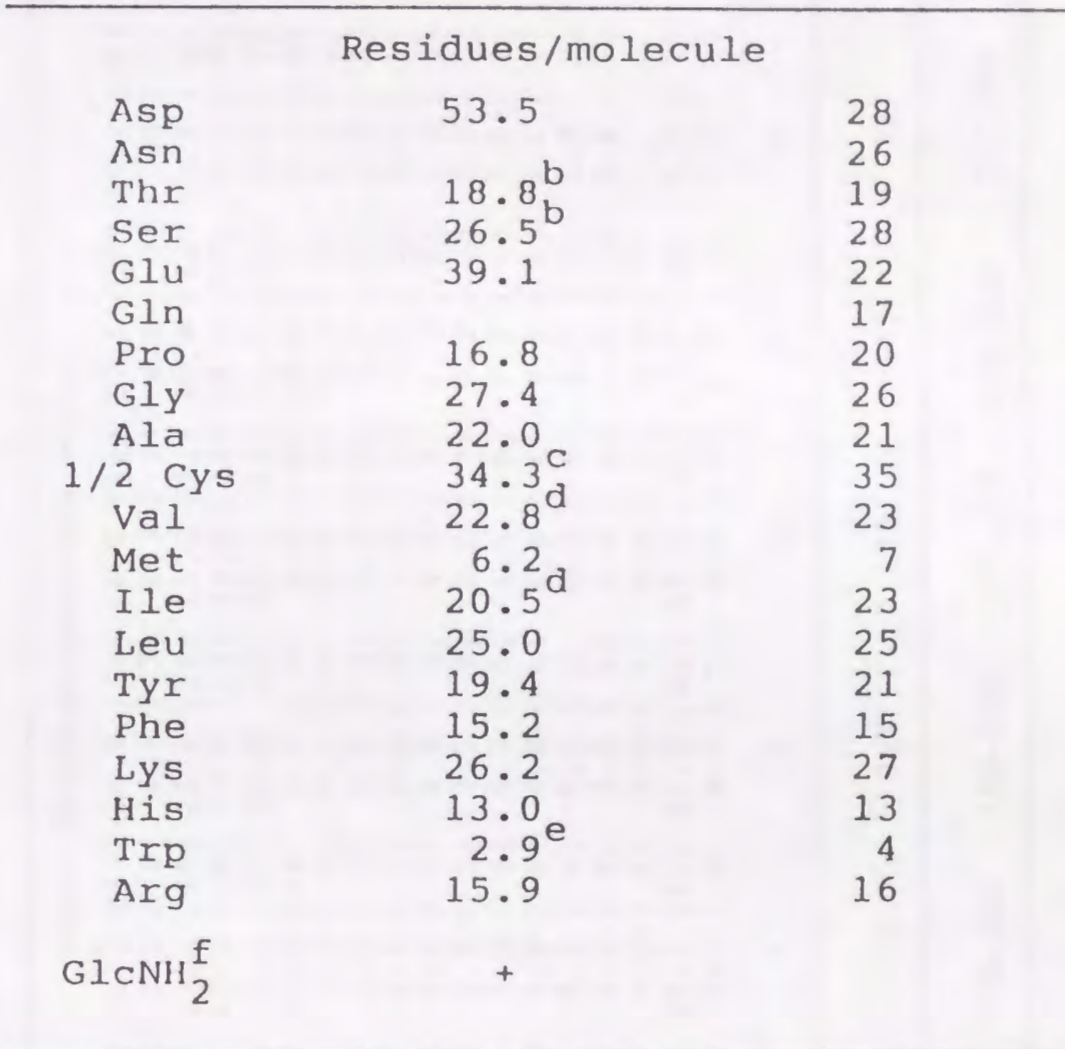

Total

416

Average values obtained from 24,48 , and 72 h hydrolyses with $5.7 \mathrm{~N} \mathrm{HCl}$. Extrapolated values to zero time. ${ }^{C}$ Determined as cysteic acid after performic acid oxidation.

draken from 72 h hydrolysis.

ebtained from 24 h hydrolysis with $3 \mathrm{~N}$ mercaptoethanesulfonic acid.

$\mathrm{f}_{\mathrm{Glucos}}$ amine. 
Table II

Amino acid compositions of cyanogen bromide peptides derived from Pe-HR1Ba

\begin{tabular}{|c|c|c|c|c|c|c|c|c|c|c|c|}
\hline $\begin{array}{l}\text { Amino } \\
\text { acid }\end{array}$ & MI & M2 & M3 & $M 3-4-5$ & M4-5 & M5 & M5-DP & M6 & M7 & $M 7^{\prime}$ & M7* \\
\hline \multicolumn{12}{|c|}{ - Residues/molecule } \\
\hline Asp & $13.1(13)$ & $3.2(3)$ & $2.9(3)$ & $21.2(19)$ & $16.9(16)$ & $16.0(16)$ & 17.5 (15) & $14.1(14)$ & $5.0(5)$ & $5.1(5)$ & $4.9(5)$ \\
\hline Thr & $7.5(8)$ & $1.1(1)$ & $0.9(1)$ & $7.2(8)$ & $6.4(7)$ & $6.3(7)$ & $6.4(7)$ & $1.0(1)$ & $1.0(1)$ & $1.2(1)$ & $0.9(1)$ \\
\hline Ser & $6.6(7)$ & $2.0(2)$ & $1.3(1)$ & $13.2(15)$ & $11.8(14)$ & $11.0(13)$ & $10.4(12)$ & $1.9(2)$ & $1.1(1)$ & $1.2(1)$ & $1.6(2)$ \\
\hline Glu & $9.4(9)$ & $2.2(2)$ & & $19.6(19)$ & $19.5(19)$ & 18.5 (19) & $18.8 \quad(19)$ & $7.3(7)$ & $2.2(2)$ & $2.0 i 2 j$ & $2.0(2)$ \\
\hline Pro & $2.2(2)$ & $2: 1 ; 2)$ & $2.3(2)$ & $14.1(14)$ & $10.8(12)$ & $10.7(11)$ & $10.5(11)$ & $2.2(2)$ & & & \\
\hline Gly & $5.4(5)$ & $1.4(1)$ & $4.7(5)$ & $13.4(14)$ & $9.5(9)$ & 9.7 (9) & 10.8 (9) & $4.3 i 4)$ & $2.2(2)$ & $2.1(2)$ & $1.9(2)$ \\
\hline Ala & $5.9(6)$ & $1.1(1)$ & & $9.3(9)$ & 9.1 ( 9 ) & 8.7 (9) & 9.0 (9) & $3.2(3)$ & $2.0(2)$ & $2.1(2)$ & $1.9(2)$ \\
\hline $\operatorname{Val}_{\mathrm{b}}$ & $8.8(9)$ & $4.7(5)$ & $0.5(0)$ & $3.9(3)$ & $4.0(3)$ & $3.2(3)$ & $3.2(3)$ & $2.3(2)$ & $4.1(4)$ & $4.0(4)$ & $3.8(4)$ \\
\hline Met ${ }^{\circ}$ & $0.6(1)$ & $1.6(2)$ & $0.8(1)$ & $1.9 i 3 i$ & $0.8 i 2 j$ & 1.1 ( 1) & 0.7 ( 1$)$ & $0.6 i 1)$ & & & \\
\hline Ile & $11.2(12)$ & $2.7(3)$ & $1.8(2)$ & $6.5(7)$ & $4.5(5)$ & $4.4(5)$ & $4.2(4)$ & $1.0(1)$ & & & \\
\hline Leu & 12.8 (13) & $1.2(1)$ & $1.1(1)$ & $9.7 i 8 i$ & $7.2(7)$ & $7.1(7)-$ & $-7.2(7)$ & $3.0(3)$ & & & \\
\hline Tyr & $4.9(5)$ & $1.1(1)$ & & $7.3(6)$ & $6.3(6)$ & $6.1(6)$ & $7.1(6)$ & $7.8(8)$ & & $1.0(1)$ & $0.8(1)$ \\
\hline Phe & 3.0 ( 3) & & $1.1(1)$ & $6.8(5)$ & $4.8 i 4 j$ & $4.6(4)$ & $5.5(4)$ & $6.8(7)$ & & & \\
\hline Lys & $8.9 ; 9)$ & $1.1(1)$ & & $8.1(6)$ & $6.6 i 6)$ & $6.2(6)$ & $6.9(6)$ & $8.0(8)$ & $2.0(2)$ & $3.0(3)$ & $2.6(3)$ \\
\hline His & $3.6 d(4)$ & $0.8(1)$ & $1.8(2)$ & $4.5(6)$ & $3.8(4)$ & $3.3(4)$ & $3.5(4)$ & $0.8(1)$ & $1.1(1)$ & $1.1(1)$ & $1.0(1)$ \\
\hline $\operatorname{Trp} p_{c}$ & n.d.di (3) & & & n.d.( 1$)$ & n.d.( 1 ) & n.d.( i) & n.d.( 1 ) & & & & \\
\hline $\operatorname{Pec}^{C}$ & $1.1(1)$ & $0.9(1)$ & $2.6(3)$ & 18.7 (23) & $17.8(20)$ & $17.3(20)$ & $17.7(20)$ & $6.5(7)$ & $2.7(3)$ & $2.4(3)$ & $2.4(3)$ \\
\hline Arg & $7.0(7)$ & $1.1(1)$ & & $3.7(5)$ & $4.9(5)$ & $4.8(5)$ & $5.0(5)$ & $2.0(2)$ & $1.0(1)$ & $1.0(1)$ & $1.0(1)$ \\
\hline $\mathrm{GICNH}_{2}^{\mathrm{e}}$ & + & & & + & \multicolumn{3}{|r|}{+} & + & & & \\
\hline Total & 117 & 28 & 22 & 171 & 149 & \multicolumn{2}{|r|}{143} & 73 & 24 & 26 & 27 \\
\hline & \multirow[b]{2}{*}{$1-117$} & \multirow[b]{2}{*}{$118-145$} & \multirow[b]{2}{*}{$146-167$} & \multirow[b]{2}{*}{$146-316$} & \multirow[b]{2}{*}{$168-316$} & \multirow[b]{2}{*}{$171-316$} & \multirow[b]{2}{*}{$174-316$} & \multirow[b]{2}{*}{$317-389$} & & \multirow[b]{2}{*}{$390-415$} & \multirow{2}{*}{$390-416$} \\
\hline Position & & & & & & & & & $390-413$ & & \\
\hline Yield (z) & 44 & 24 & 48 & 6 & 6 & 23 & 8 & 54 & 27 & 16 & 5 \\
\hline
\end{tabular}

avalues in parentheses are from the sequence data. betermined as homoserine plus homoserine lactone.

S-pyridylethylcysteine. ${ }^{\text {Not determined. }}$ Glucosamine. 
Table I I

Summary of amino acid sequences of fragments derived from HRIB

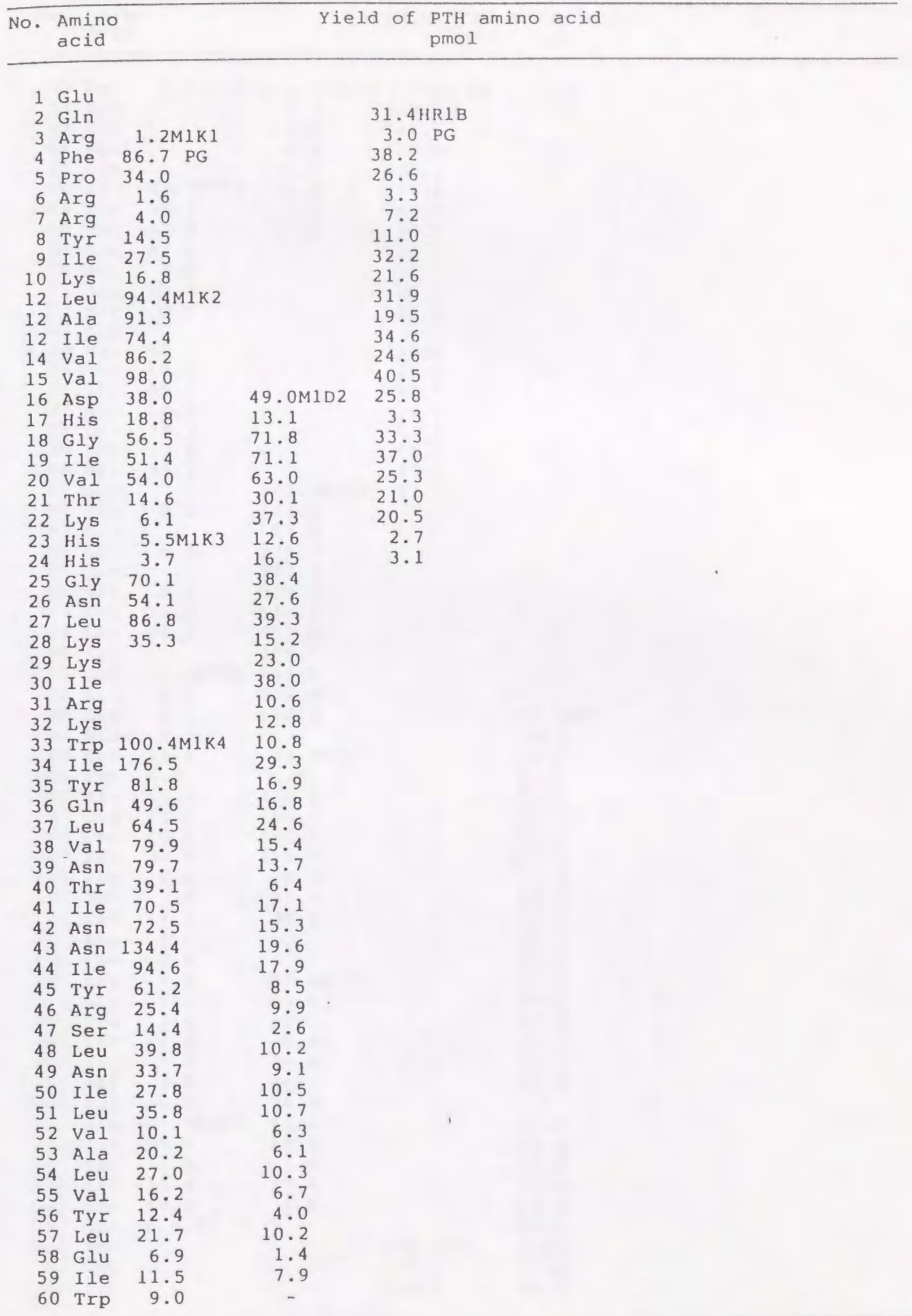




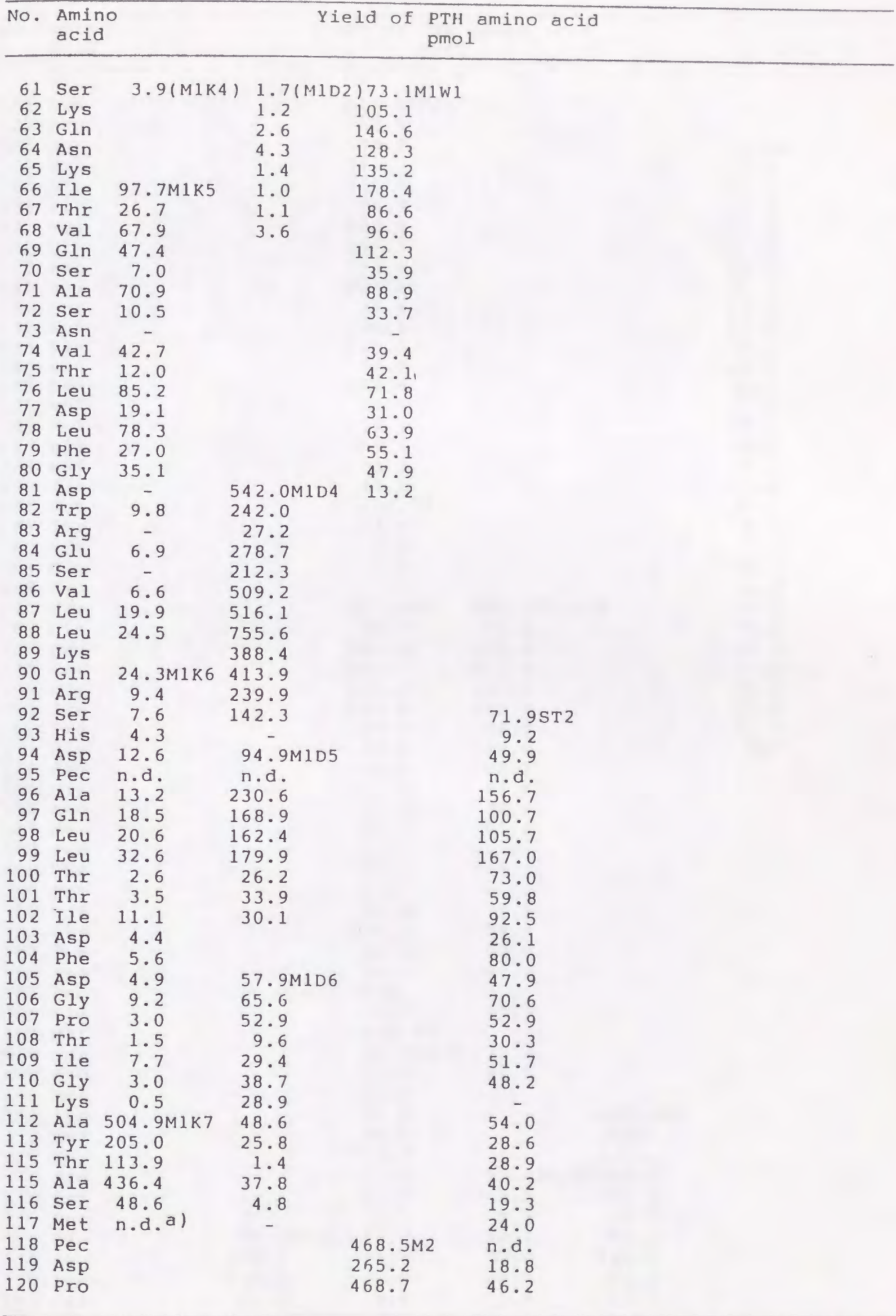


(continued-2)

Table III

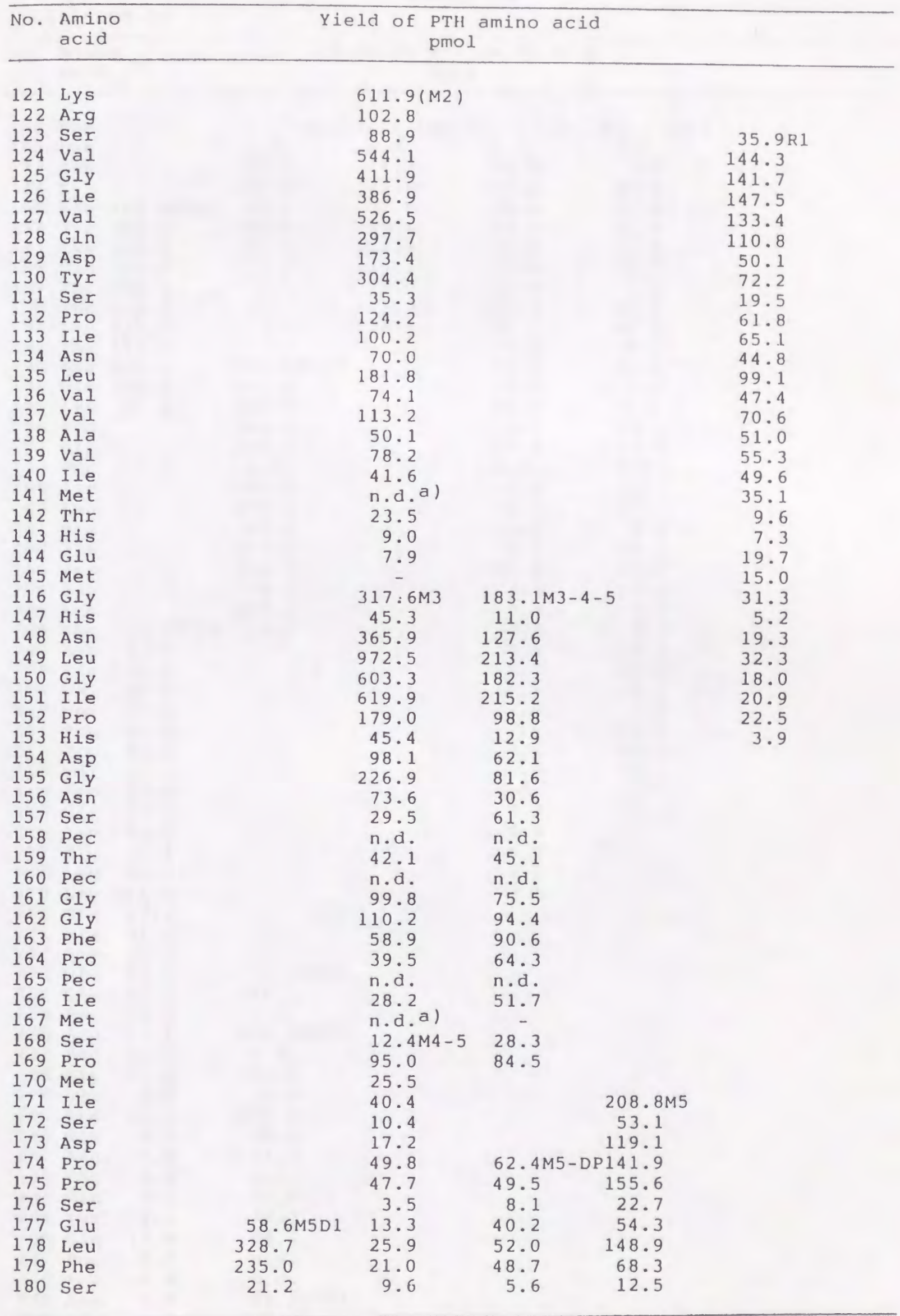


(continued-3)

Table III

\begin{tabular}{|c|c|c|c|c|c|c|}
\hline No. & $\begin{array}{l}\text { Amin } \\
\text { acid }\end{array}$ & & Yiel & d of & amino & \\
\hline 181 & Asn & & $-(M 5 D 1)$ & -11 & ) -1 & P) - (M5) \\
\hline 182 & Pec & & $\mathrm{n} \cdot \mathrm{d}$ & n.d. & n.d. & n.d. \\
\hline 183 & Ser & & 20.2 & 4.3 & 11.8 & 7.5 \\
\hline 184 & Lys & & 139.5 & 11.6 & 46.6 & 30.6 \\
\hline 185 & Ala & $468.8 M 5 \mathrm{~K} 1$ & 68.7 & 23.8 & 38.8 & 42.0 \\
\hline 186 & Tyr & 312.5 & 68.5 & 13.6 & 26.1 & 41.6 \\
\hline 187 & Tyr & 368.4 & 46.1 & 14.1 & 41.2 & 38.5 \\
\hline 188 & Gin & 300.8 & & 15.1 & 22.6 & 39.7 \\
\hline 189 & Thr & 229.4 & & 7.91 & 12.2 & 7.3 \\
\hline 190 & Phe & 262.2 & & 15.3 & 21.3 & 42.5 \\
\hline 191 & Leu & 237.8 & & 26.8 & 34.5 & 67.3 \\
\hline 192 & Thr & 157.2 & & & 4.5 & 6.2 \\
\hline 193 & Asp & 138.6 & $572.6 \mathrm{~K} 5 \mathrm{D} 3$ & & 25.0 & 12.9 \\
\hline 194 & His & 118.8 & 204.0 & & 5.0 & 5.2 \\
\hline 195 & Lys & 79.0 & 689.0 & & 18.6 & 14.6 \\
\hline 196 & Pro & & 384.0 & & 9.0 & 30.1 \\
\hline 197 & Gln & & 264.5 & & 12.1 & 12.4 \\
\hline 198 & Pec & & n.d. & & $\mathrm{n} \cdot \mathrm{d}$ & $\mathrm{n} \cdot \mathrm{d}$. \\
\hline 199 & Ile & & 405.1 & & 7.2 & 16.8 \\
\hline 200 & Leu & & 672.3 & & 8.6 & 29.1 \\
\hline 201 & Asn & & 202.9 & & 11.9 & 8.9 \\
\hline 202 & Ala & & 549.6 & & 11.1 & 21.7 \\
\hline 203 & Pro & & 256.2 & & 12.2 & 28.0 \\
\hline 204 & Ser & & 40.5 & & 2.7 & 2.3 \\
\hline 205 & Lys & & 209.1 & & 3.3 & 5.8 \\
\hline 206 & Thr & $13.2 \mathrm{M} 5 \mathrm{~K} 3$ & 17.0 & & 2.2 & $3 \cdot 3$ \\
\hline 207 & Asp & 23.7 & & & 6.1 & 12.3 \\
\hline 208 & Ile & 125.6 & & & 5.7 & 10.0 \\
\hline 209 & Val & 80.5 & & & 3.1 & 9.4 \\
\hline 210 & Ser & 16.8 & & & 2.0 & 2.6 \\
\hline 211 & Pro & 80.5 & & & 7.4 & 22.8 \\
\hline 212 & Pro & 85.1 & & & 7.2 & 22.5 \\
\hline 213 & Val & 47.9 & & & 3.3 & 10.1 \\
\hline 214 & Pec & 32.6 & & & $\mathrm{n} \cdot \mathrm{d}$ & $\mathrm{n} \cdot \mathrm{d}$ \\
\hline 215 & Gly & 47.3 & & & 8.3 & 13.6 \\
\hline 216 & Asn & 33.8 & & & 4.6 & \\
\hline 217 & Glu & 25.3 & & & 6.8 & \\
\hline 218 & Leu & 96.4 & & & 2.5 & \\
\hline 219 & Leu & 164.0 & & & 3.6 & \\
\hline 220 & Glu & 27.8 & & & & \\
\hline 221 & Ala & 34.2 & & & & \\
\hline 222 & Gly & 33.7 & & & & \\
\hline 223 & Glu & 23.8 & $91.5 \mathrm{M} 5 \mathrm{D} 5$ & & & \\
\hline 224 & Glu & 32.3 & 146.1 & & & \\
\hline 225 & Pec & $14 \cdot 1$ & $\mathrm{n} \cdot \mathrm{d}$ & & & \\
\hline 226 & Asp & 12.4 & $150.0 M 5 D 6$ & & & \\
\hline 227 & Pec & 21.0 & $\mathrm{n} \cdot \mathrm{d}$ & & & \\
\hline $\begin{array}{l}228 \\
229\end{array}$ & $\begin{array}{l}\text { Gly } \\
\text { Ser }\end{array}$ & $\begin{array}{r}21.9 \\
3.0\end{array}$ & $\begin{array}{r}185.0 \\
38.4\end{array}$ & & & \\
\hline $\begin{array}{l}229 \\
230\end{array}$ & $\begin{array}{l}\text { Ser } \\
\text { Pro }\end{array}$ & $\begin{array}{r}3.0 \\
12.1\end{array}$ & 135.9 & & & \\
\hline 231 & Glu & 7.3 & 79.6 & & & \\
\hline 232 & Asn & 6.6 & 74.3 & & & \\
\hline 233 & Pec & 6.5 & $\mathrm{n} \cdot \mathrm{d}$. & & & \\
\hline 234 & Gln & 7.0 & 82.9 & & & \\
\hline 235 & Tyr & $\begin{array}{r}4.3 \\
14.8\end{array}$ & $\begin{array}{l}73.7 \\
99.2\end{array}$ & & & \\
\hline $\begin{array}{l}236 \\
237\end{array}$ & $\begin{array}{l}\text { Gln } \\
\text { Pec }\end{array}$ & $\begin{array}{r}14.8 \\
9.8\end{array}$ & $\begin{array}{l}99.2 \\
\text { n.d. }\end{array}$ & & & \\
\hline 238 & Pec & 10.0 & $\mathrm{n} \cdot \mathrm{d}$. & & & \\
\hline 239 & Asp & 3.9 & 82. 3M5D7 & & & \\
\hline 240 & Ala & 6.3 & 340.4 & & & \\
\hline
\end{tabular}


(continued-4)

\begin{tabular}{|c|c|c|c|}
\hline No. & $\begin{array}{l}\text { Amino } \\
\text { acid }\end{array}$ & Yield of & $\begin{array}{l}\text { PTH amino } \\
\text { pmol }\end{array}$ \\
\hline
\end{tabular}

241 Ala 1

242 Ser

243 Pec

244 Lys

245 Leu

246 His

247 Ser

248 Trp 257.5

249 Val 263.3

250 Lys 152.6

251 Pec 59.6M5K5

252 Glu 48.6

253 Ser 13.5

254 Gly 68.4

255 Glu 39.3

256 Pec 101.3

257 Pec 100.3

258 Asp 40.6

259 Gln 36.9

260 PeC 74.0

261 Arg 26.7

262 Phe 56.6

263 Arg 31.9

264 Thr

265 Ala

266 Gly

267 Thr

$268 \mathrm{Glu}$

269 Pec

270 Arg

271 Ala

272 Ala

273 Glu

274 Ser

$275 \mathrm{Glu}$

276 Pec

277 Asp

278 Ile

279 Pro

$280 \mathrm{Glu}$

281 Ser

282 Pec

283 Thr

284 Gly

285 GIn

286 Ser

287 Ala

288 Asp

289 Pec

290 Pro

291 Thr

292 Asp

293 Arg

294 Phe

$295 \mathrm{His}$

296 Arg

297 Asn

298 G Iy

299 Gln

300 Pro
9.1

36.7

29.0

6.9

18.5

34.3

11.9

14.1

26.6

13.0

3. 0

9.7

15.9

$9.4 \quad 162.5 M 5 D 10$

$8.2 \quad 253.8$

8. 200.0

4. $3 \quad 106.6$

1. $0 \quad 35.3$

7.4

n.d.

51.9

$6.4 \quad 114.8$

$3.3 \quad 89.8$

$0.8 \quad 24.3$

$3.8 \quad 27.1$

3. 3

3. 4

11. $7 \mathrm{M} 5 \mathrm{D} 12$

25.9

58.7

3.8

10.7

22.6

38.8

35.4

17.0
$1743.7 \mathrm{ST} 3$

152.6

927.4

1741.9

1359.2

824.5

697.9

n.d.

41.2

$810.9 \mathrm{ST} 4 \quad 372.2 \mathrm{ST} 4-5$

$764.1 \quad 358.2$

$290.1 \quad 141.1$

$185.8 \quad 60.4$

$298.3 \quad 130.6$

n.d. $\quad 195.8$

$171.3 \quad 91.7$

$380.4 \quad 205.9$

$224.6 \quad 104.5$

$141.6 \quad 79.8$

$81.0 \quad 32.2$

n.d. $\quad 64.8$

$115.8 \quad 54.2$

$134.4 \quad 94.1$

$122.9 \quad 60.1$

$50.9 \quad 29.8$

$106.8 \quad 80.8$

$51.0 \quad 37.3$

n.d. $\quad 54.6$

$50.2 \quad 44.8$

$31.5 \quad 19.2$

$32.6 \quad 38.2$

16.69 .6

$37.7 \quad 41.9$

$4.0 \quad 4.8$

$16.5 \quad 15.5$

27.6 ST 5

71.8

58.6

58.1

24.4 
(continued -5 )

Table III

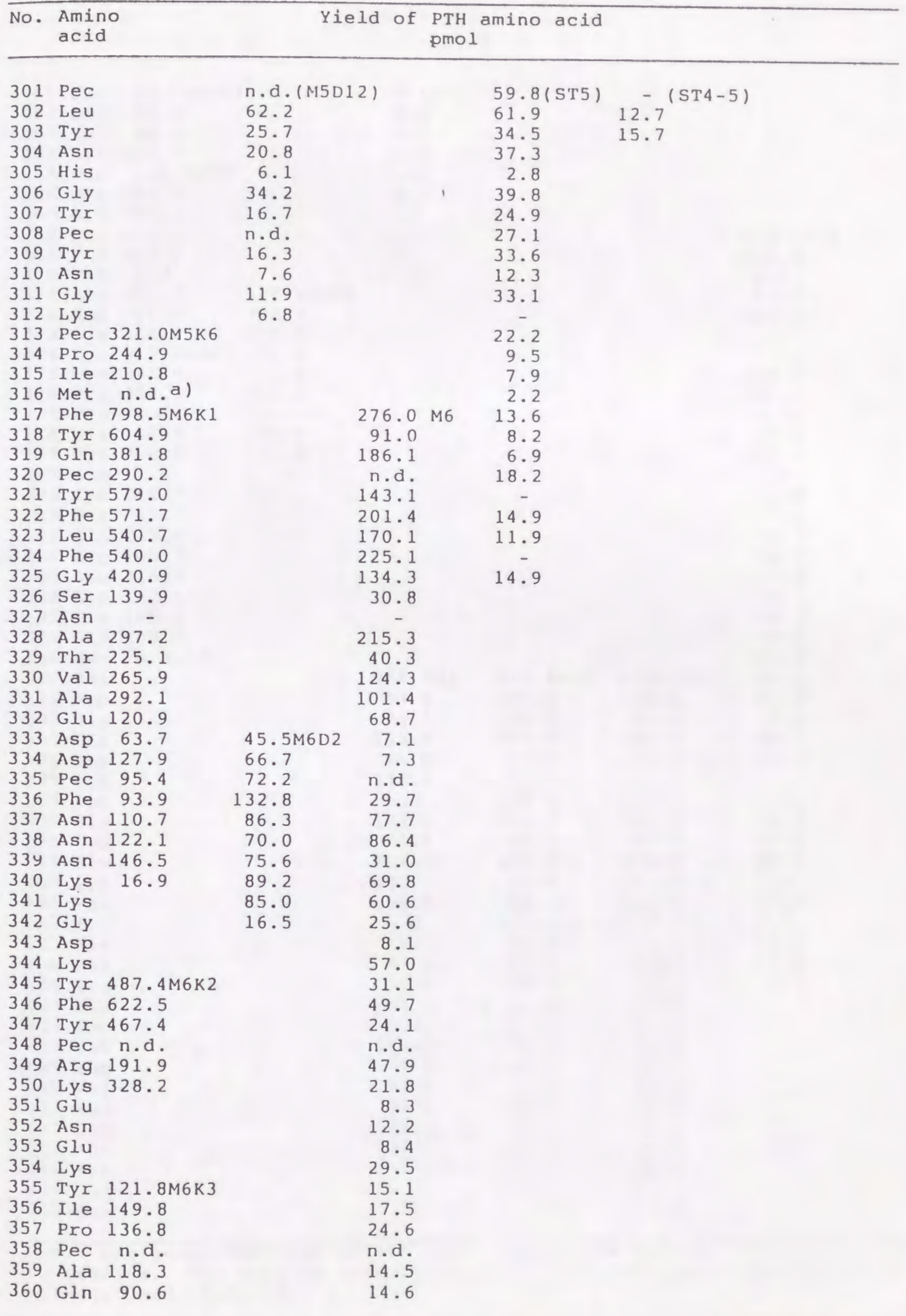


(continued-6)

Table II I

\begin{tabular}{|c|c|c|c|c|c|c|c|}
\hline No. & $\begin{array}{l}\text { Amin } \\
\text { acid }\end{array}$ & & Yi & ld of & amino aci & & \\
\hline 361 & Glu & $52.7(\mathrm{M} 6 \mathrm{~K} 3$ & & $9.1(\mathrm{M}$ & & & \\
\hline 362 & Asp & 35.0 & & 4.8 & & & \\
\hline 363 & Val & 68.3 & & 7.1 & & & \\
\hline 364 & Lys & 127.9 & & 6.8 & & & \\
\hline 365 & $\mathrm{PeC}$ & n.d.M6K4 & & n.d. & & & \\
\hline 366 & Gly & 440.7 & & 8.8 & & & \\
\hline 367 & Arg & 157.7 & & - & & & \\
\hline 368 & Leu & 451.1 & & 16.5 & & & $453.3 \mathrm{ST} 6$ \\
\hline 369 & Phe & 459.6 & & 3.1 & & & 448.8 \\
\hline 370 & $\mathrm{PeC}$ & n.d. & & n.d. & & & n.d. \\
\hline 371 & Asp & 172.3 & $147.4 M 6 D 6$ & & & & 87.3 \\
\hline 372 & Asn & 217.5 & 244.1 & & & & 182.0 \\
\hline 373 & Lys & 158.6 & 350.8 & & & & - \\
\hline 374 & Lys & $312.9 \mathrm{M} 6 \mathrm{K5}$ & 375.6 & & & & - \\
\hline 375 & Tyr & 291.9 & 259.6 & & & & 228.4 \\
\hline 376 & Pro & 352.7 & 287.2 & & & & 191.1 \\
\hline 377 & Pec & n.d. & $n \cdot d$. & & & & n.d. \\
\hline 378 & His & 50.4 & 60.4 & & & & 17.1 \\
\hline 379 & Tyx & 259.0 & 97.6 & & & & 122.2 \\
\hline 380 & Asn & - & - & & & & - \\
\hline 381 & Tyr & 240.7 & 186.2 & & & & 176.8 \\
\hline 382 & Ser & 45.7 & 31.9 & & & & 53.4 \\
\hline 383 & Glu & 129.8 & 30.7 & & & & 56.3 \\
\hline 384 & Asp & 105.7 & & & & & 52.9 \\
\hline 385 & Leu & 316.8 & & & & & 84.4 \\
\hline 386 & Asp & 132.4 & & & & & 78.1 \\
\hline 387 & Phe & 148.3 & & & & & 71.2 \\
\hline 388 & Gly & 109.9 & & & & & 58.0 \\
\hline 389 & Met & n.d.a) & & & & & 40.5 \\
\hline 390 & Val & & & $574.9 M 7$ & $379.4 M 7^{\prime}$ & $466.1 M 7 "$ & 57.1 \\
\hline 391 & Asp & & & 63.6 & 194.8 & 139.9 & 41.9 \\
\hline 392 & His & & & 24.1 & 45.4 & 29.2 & 5.6 \\
\hline 393 & Gly & & & 255.3 & 179.8 & 156.3 & 30.7 \\
\hline 394 & Thr & & & 44.0 & 27.4 & 123.6 & 41.0 \\
\hline 395 & Lys & & & 132.3 & 149.9 & 214.8 & - \\
\hline 396 & $\mathrm{PeC}$ & & & n.d. & 168.5 & 133.9 & n.d. \\
\hline 397 & Ala & & & 132.3 & 141.5 & 244.6 & 14.0 \\
\hline 398 & Asp & & & 51.9 & 87.7 & 45.7 & 10.6 \\
\hline 399 & Gly & & & 104.0 & 105.8 & 104.9 & 24.4 \\
\hline 400 & Lys & & & 130.7 & 95.4 & 107.9 & - \\
\hline 401 & Val & & & 156.4 & 92.5 & 139.0 & 11.0 \\
\hline 402 & Pec & & & $\mathrm{n} \cdot \mathrm{d}$. & 127.5 & 113.0 & n.d. \\
\hline 403 & Ser & & & 18.2 & 19.2 & 41.6 & 5.4 \\
\hline 404 & Asn & & & 57.8 & 52.2 & 91.0 & 5.9 \\
\hline 405 & Arg & & & 33.7 & 43.2 & 24.6 & 1.7 \\
\hline 406 & Gln & & & 64.2 & 52.4 & 92.4 & \\
\hline 407 & Pec & & & n.d. & 71.8 & 86.5 & \\
\hline 408 & Val & & & 106.8 & 43.2 & 51.6 & \\
\hline 409 & Asp & & & 35.3 & 39.2 & 22.9 & \\
\hline 410 & Val & & & 93.5 & 55.3 & 63.6 & \\
\hline 411 & Asn & & & 36.1 & 38.7 & 39.3 & \\
\hline 412 & Glu & & & 22.8 & 28.2 & 39.1 & \\
\hline 413 & Ala & & & 12.5 & 37.9 & 54.8 & \\
\hline 414 & Tyr & & & & 26.7 & 36.3 & \\
\hline 415 & Lys & & & & 7.7 & 27.7 & \\
\hline 416 & Ser & & & & & 9.9 & \\
\hline
\end{tabular}

n.d.: not quantitatively determined. - not identified. addentified as homoserine. The peptide nomenclature is described under "Materials and Methods" and "Results". 
Table IV

Amino acid compositions of lysyl endopeptidase peptides derived from $\mathrm{Ml}^{\mathrm{a}}$

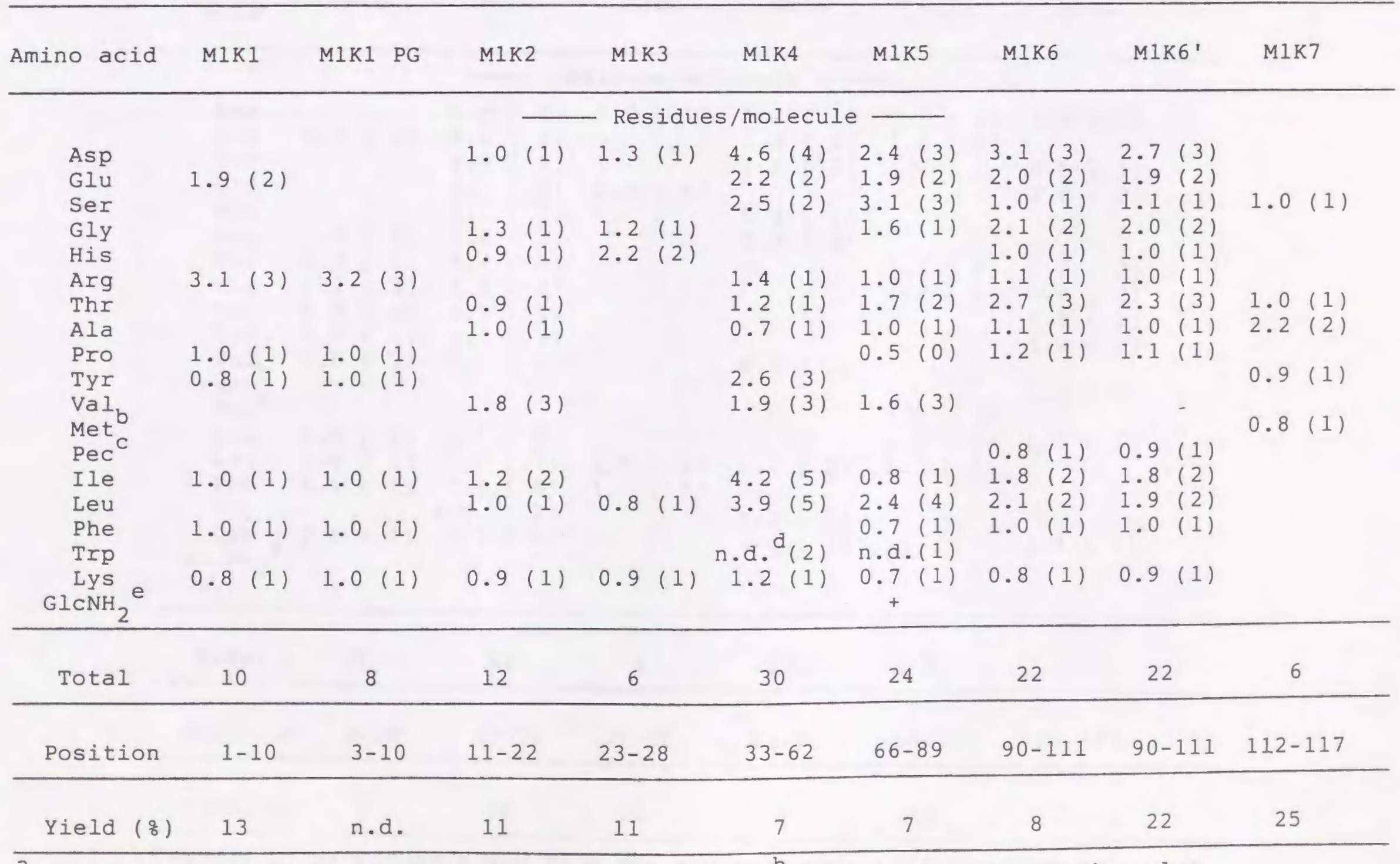

${ }^{a}$ Velues in parentheses are from the sequence data. ${ }^{b}$ Determined as homoserine plus

homoserine lactone. C S-pyridylethylcysteine. Not determined. Glucosamine. 
Table v

Amino acid compositions of endoproteinase Asp-N peptides derived from $\mathrm{Ml}^{\mathrm{a}}$

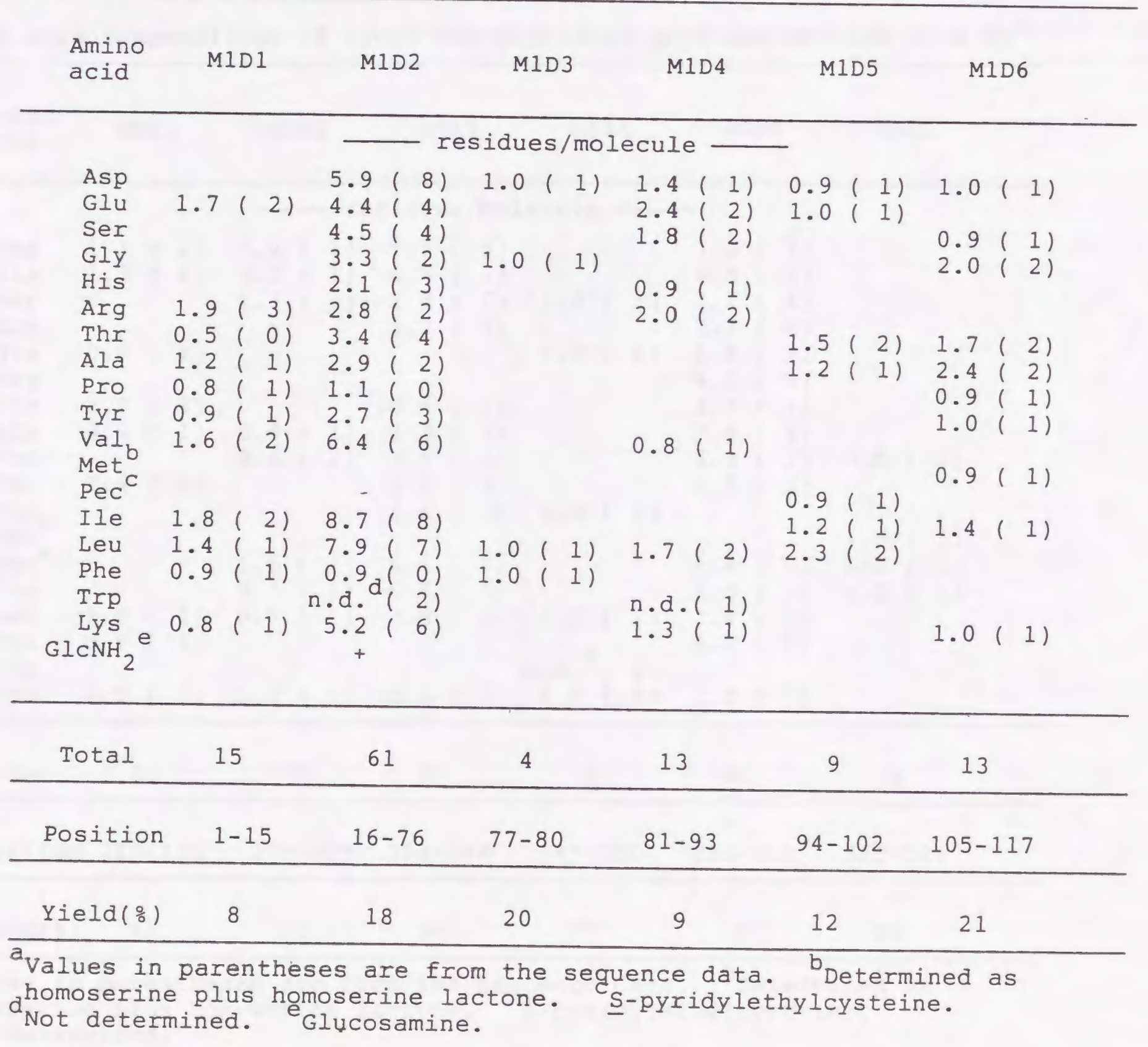


Table VI

Amino acid compositions of lysyl endopeptidase peptides derived from $\mathrm{M}^{\mathrm{a}}$

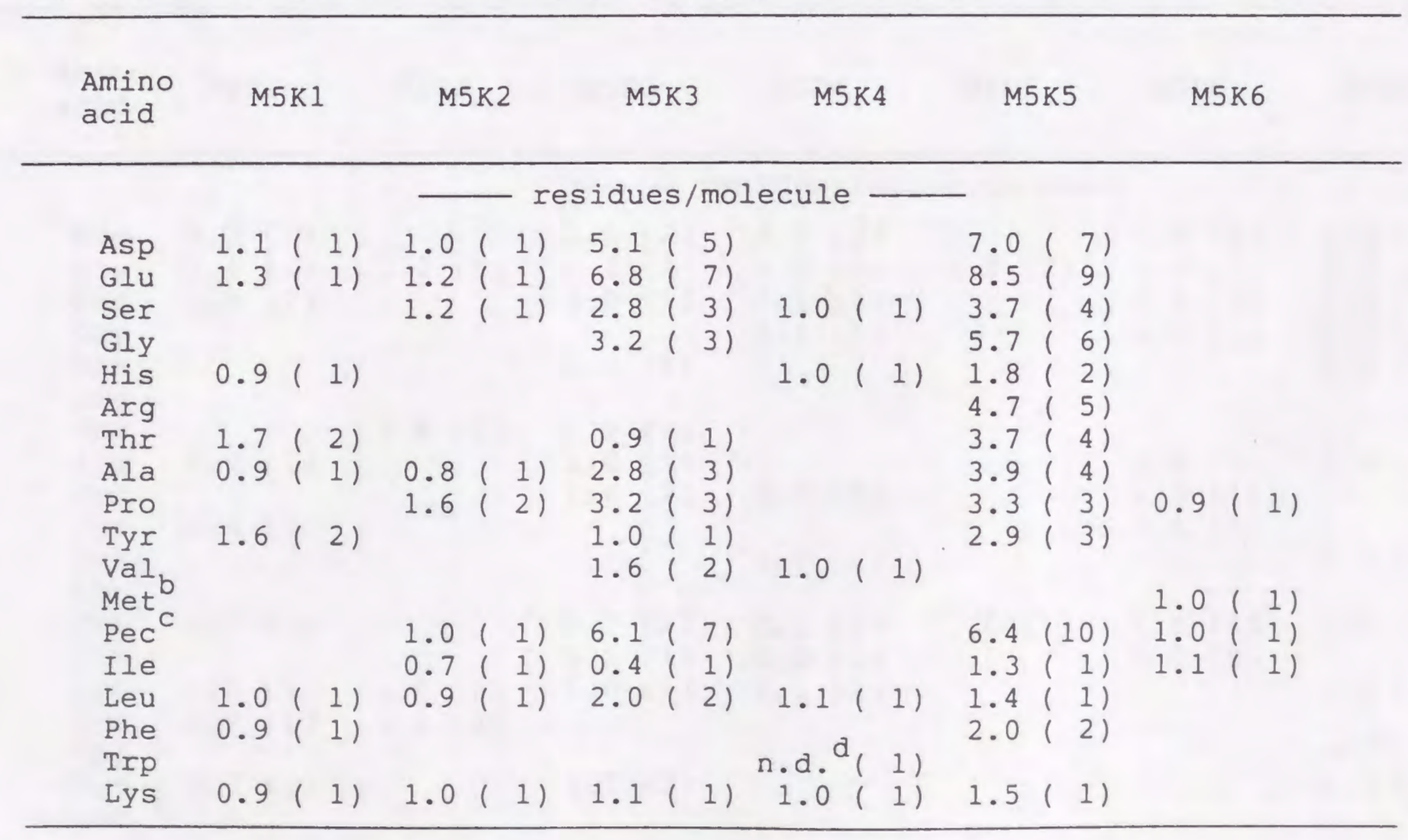

\begin{tabular}{lcccccc} 
Total & 11 & 10 & 39 & 6 & 62 & 4 \\
\hline $\begin{array}{l}\text { Position } \\
185-195\end{array}$ & $196-205$ & $206-244$ & $245-250$ & $251-312$ & $313-316$ \\
\hline Yield $(\%)$ & 45 & 72 & 34 & 37 & 7 \\
\hline $\begin{array}{l}\text { Values in parentheses are from the sequence data. Determined as } \\
\text { homoserine plus homoserine lactone. } \\
\text { dNot determined. }\end{array}$
\end{tabular}


Table VII

Amino acid compositions of endoproteinase Asp-N peptides derived from $\mathrm{M}^{\mathrm{a}}$

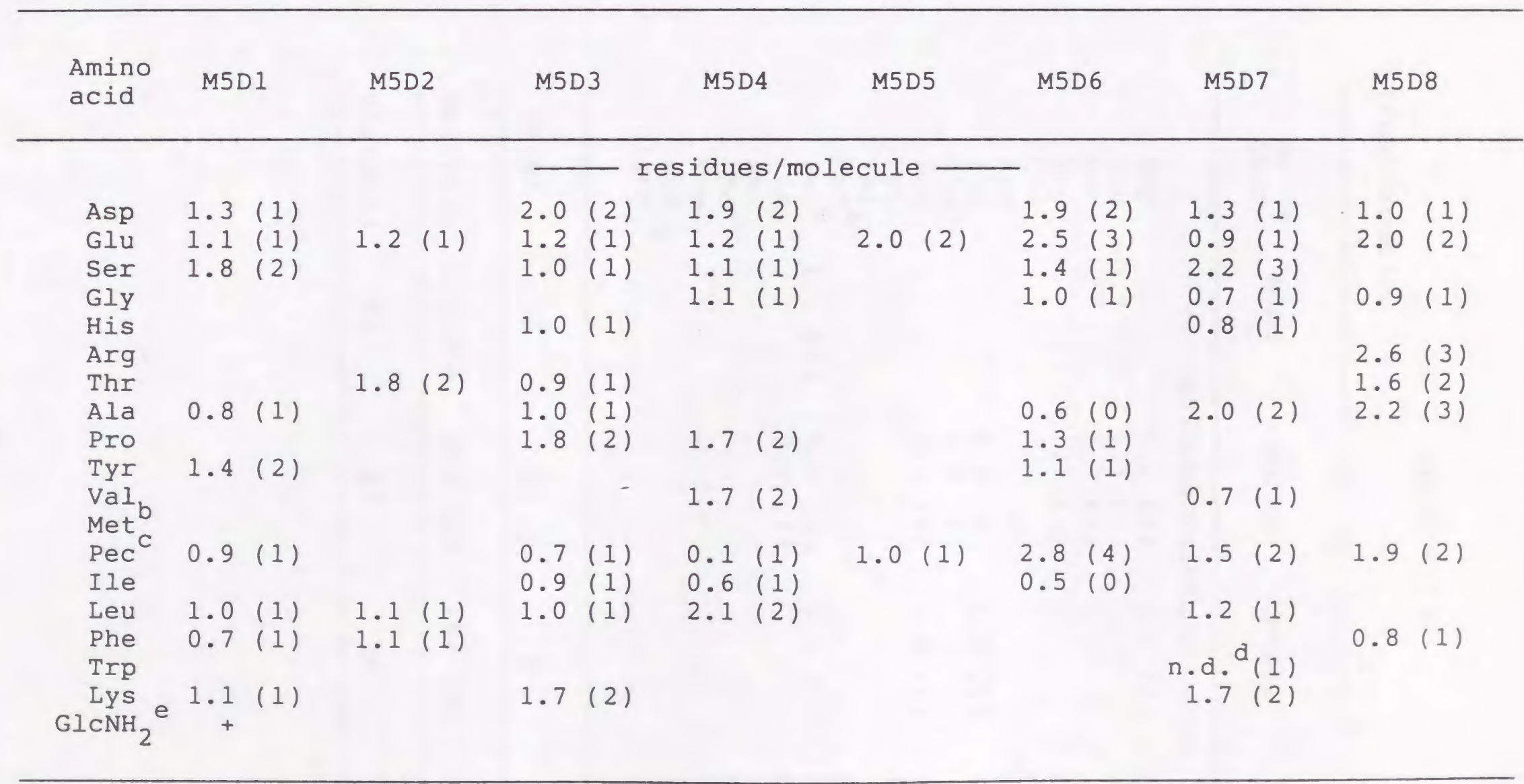

\begin{tabular}{|c|c|c|c|c|c|c|c|c|}
\hline Total & 11 & 5 & 14 & 13 & 3 & 13 & 16 & 15 \\
\hline Position & $177-187$ & $188-192$ & $193-206$ & $207-219$ & $223-225$ & $226-238$ & $239-254$ & $258-272$ \\
\hline Yield( & 6 & 19 & 19 & 27 & 43 & 26 & 11 & 30 \\
\hline
\end{tabular}

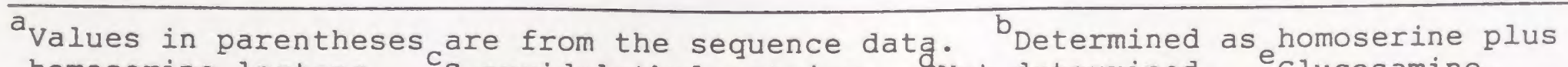
homoserine lactone. ${ }^{C}$ S-pyridylethylcysteine. ${ }_{\text {Not determined. }}$ Glucosamine. 
Table VII

(continued)

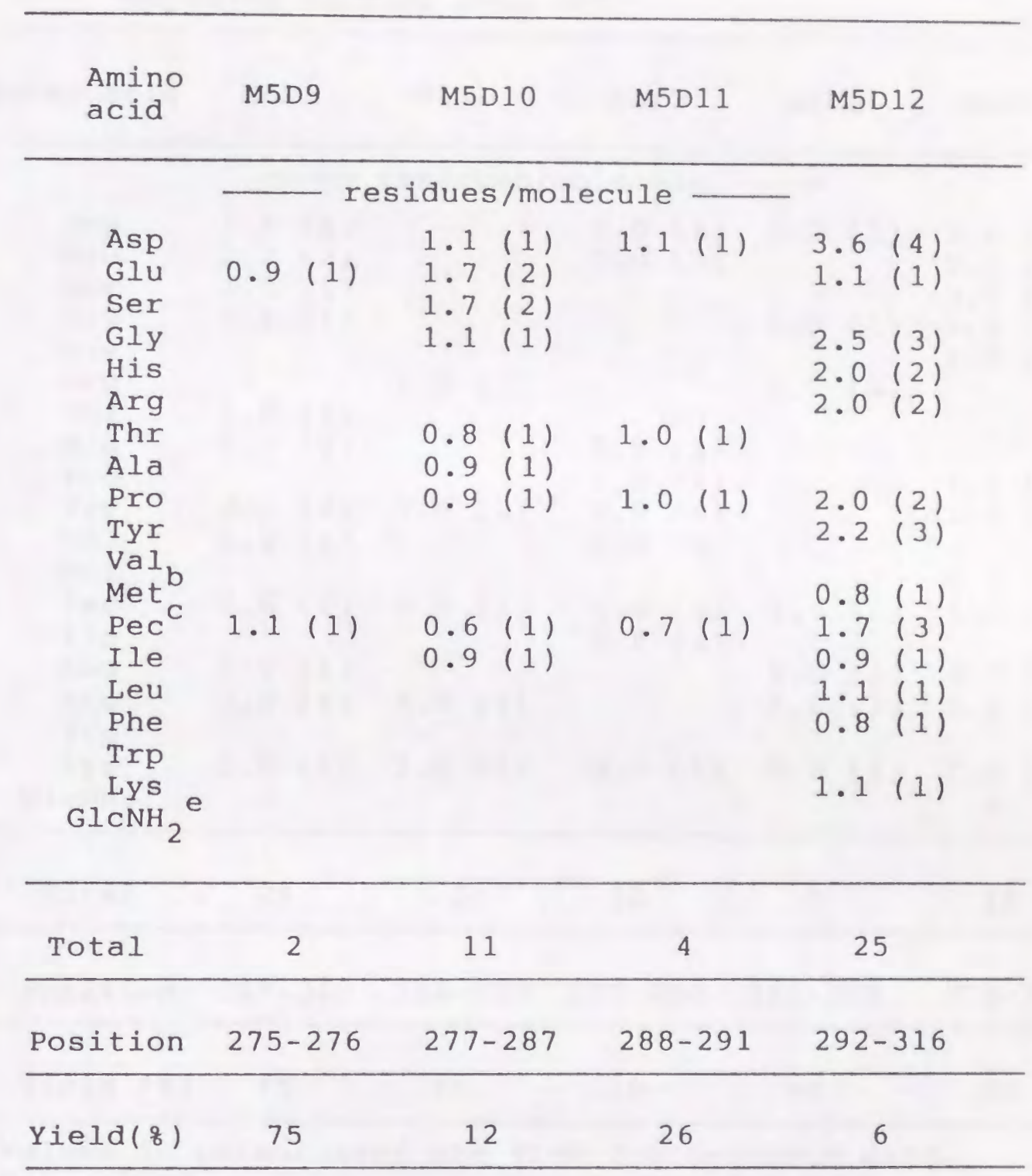


Table VIII Amino acid compositions of lysyl endopeptidase
peptides derived from $\mathrm{M}^{\mathrm{a}}$

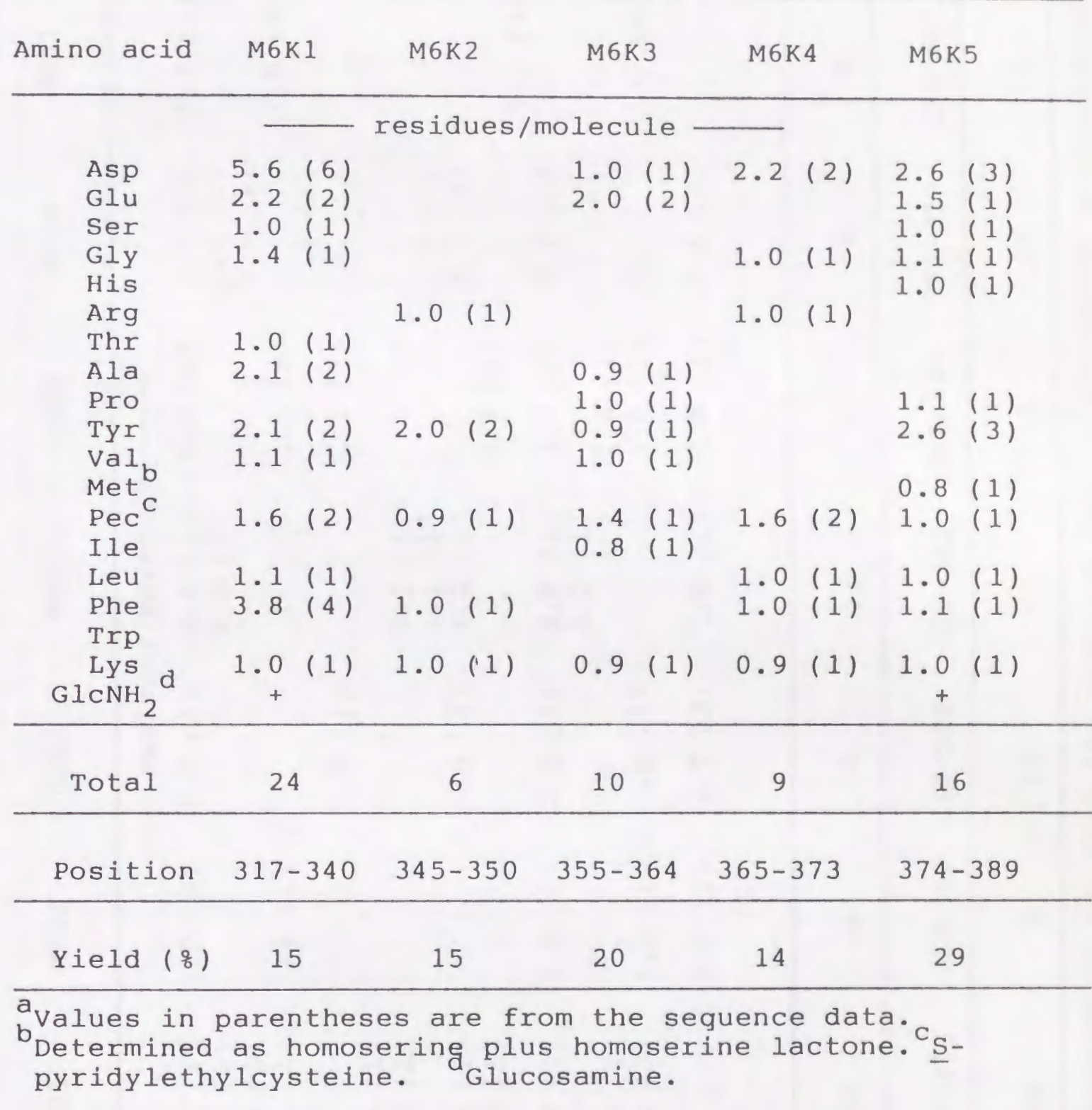


Table IX

Amino acid compositions of endoproteinase Asp-N peptides derived from $\mathrm{M}^{\mathrm{a}}$

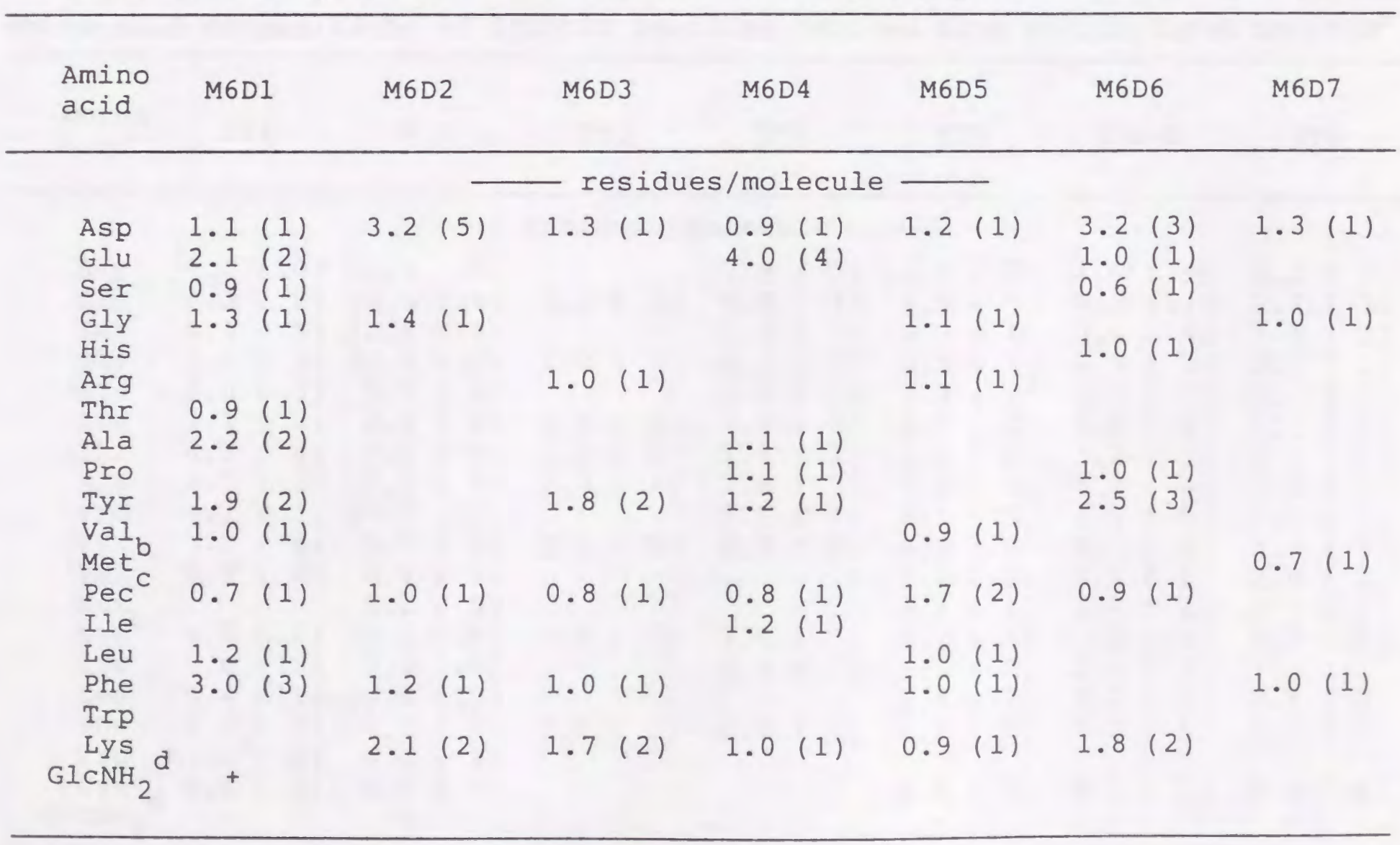

\begin{tabular}{|c|c|c|c|c|c|c|c|}
\hline Total & 16 & 10 & 8 & 11 & 9 & 13 & 4 \\
\hline Position & $317-332$ & $333-342$ & $343-350$ & $351-361$ & $362-370$ & $371-383$ & $384-387$ \\
\hline Yield ( 8 ) & 16 & 9 & 13 & 11 & 17 & 28 & 11 \\
\hline
\end{tabular}


Table $\mathrm{X}$

Amino acid compositions of triptic peptides derived from succinylated Pe-HRlB

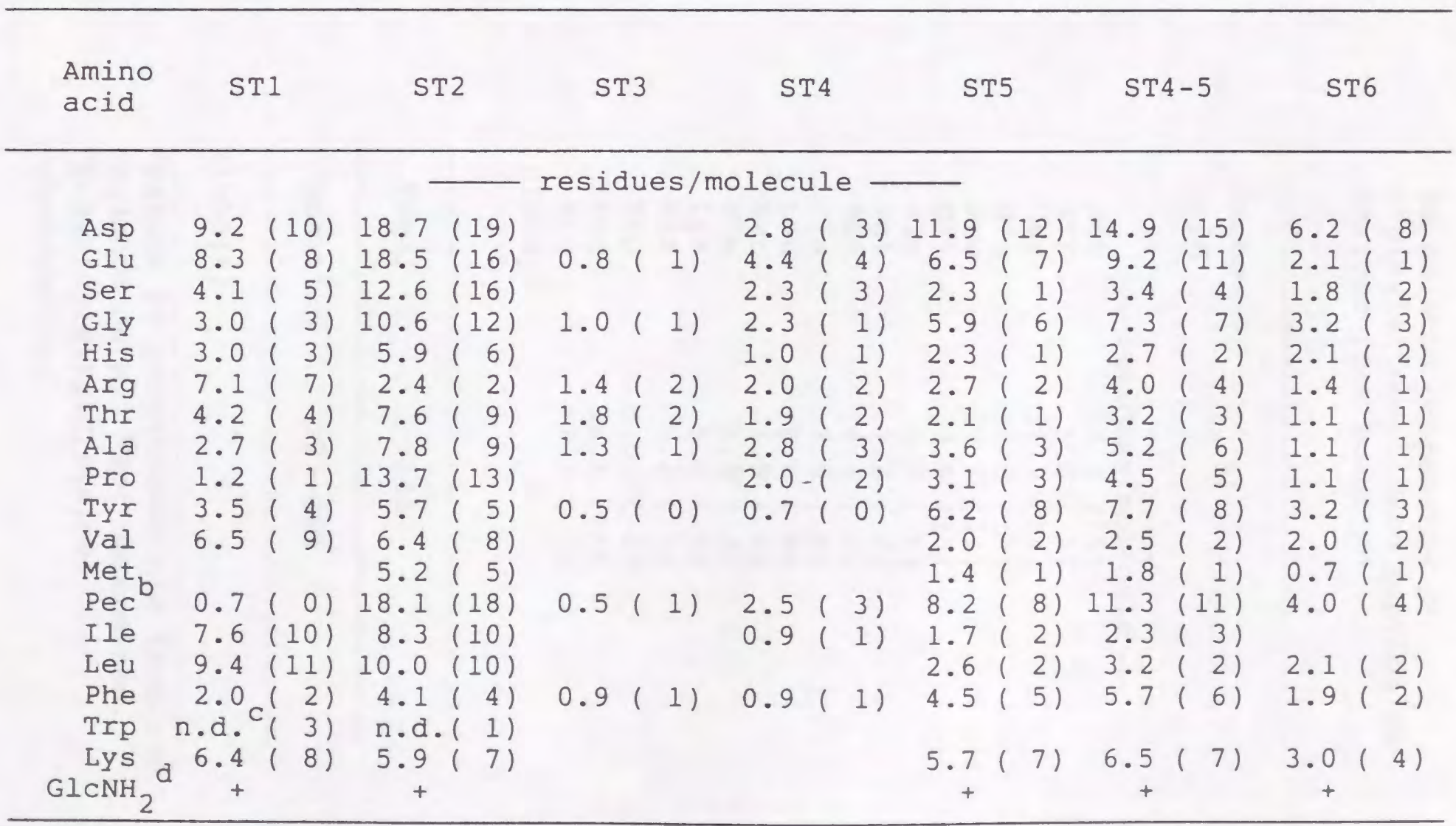

\begin{tabular}{lccccccc}
\hline Total & 91 & 170 & 9 & 26 & 71 & 97 & 38 \\
\hline Position & $1-91$ & $92-261$ & $262-270$ & $271-296$ & $297-367$ & $271-367$ & $368-405$ \\
\hline
\end{tabular}

\begin{tabular}{llllllll} 
Yield $(\%)$ & 23 & 16 & 6 & 8 & 11 & 10 & 4 \\
\hline
\end{tabular}

a Values in parentheses are from the sequence data. ${ }^{\mathrm{b}}$ s-pyridylethylcysteine. Not determined. alucosamine. 
Table XI

Amino acid compositon of

arginylendopeptidase peptide R1 derived from Pe-HR1Ba

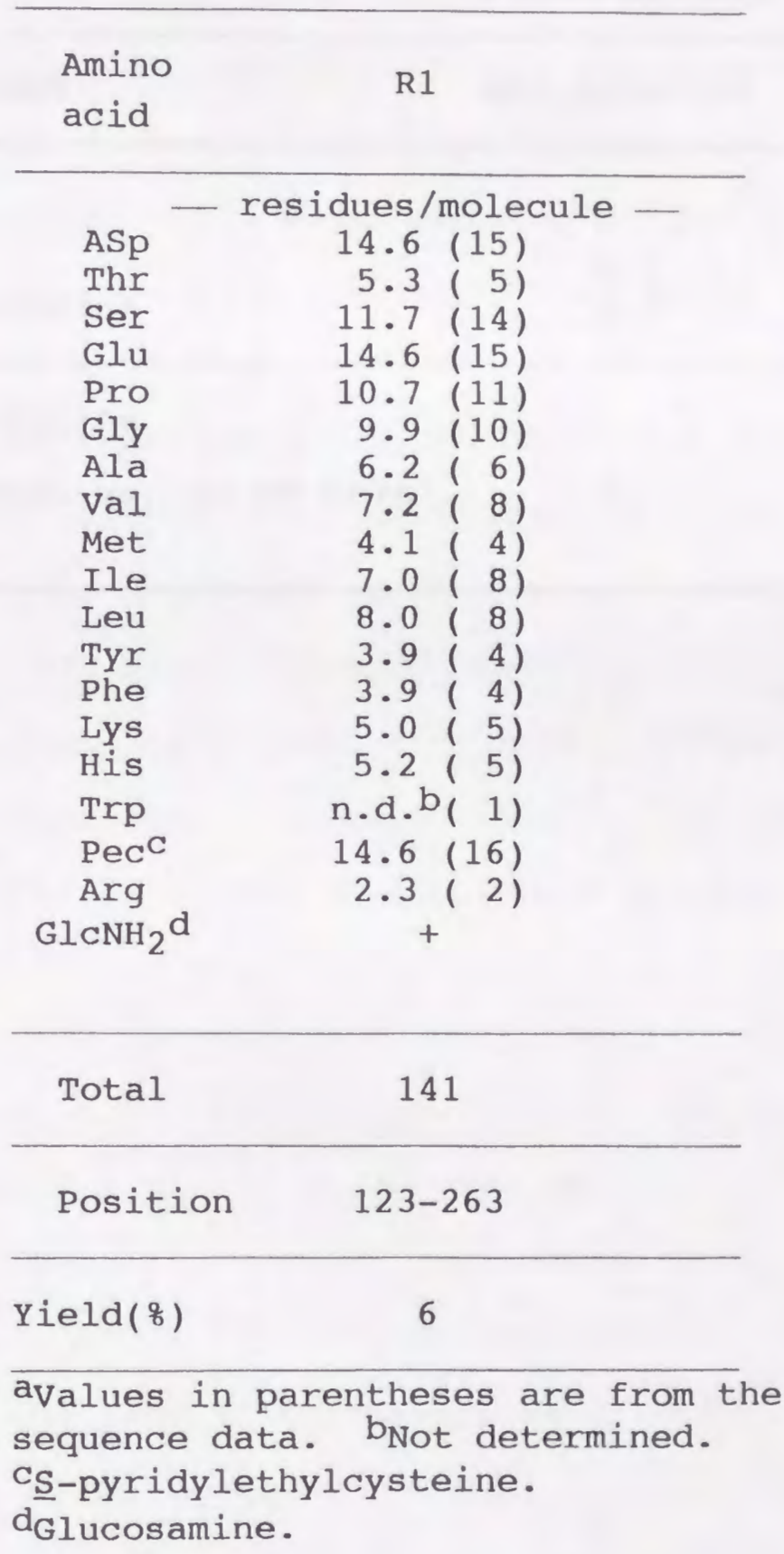


Table XII

Reactivity of the free sulfhydryl group in HRIB

mol/molecule

\section{DTNB}

None

6M Guanidine

0.1

0.9

4-vinylpyridine

6M Guanidine, $10 \mathrm{mM}$ EDTA'

$$
0.7
$$




\section{LEGEND FOR FIGURE}

Fig. 1. Complete amino acid sequence of HR1B. Residues determined by Edman degradation are written in upper case letters below the summarized sequence. Residues written in lower case letters indicate tentative identifications deduced from the amino acid compositions of the peptides. Dashes indicate unidentified residues. The peptide nomenclature is described under ' Materials and Methods" and "Results". O indicate sugar chains linked to Asn residue. $<$ E denotes 5-pyrrolidone-2-carboxylic acid.

Fig. 2. HPLC of CNBr peptides derived from Pe-HR1B. A part of the digest was subjected to HPLC as described in Materials and Methods. A Vydac 214 TP5415 (0.46 x $15 \mathrm{~cm}$ ) column was used. Peptides M4-5 and M5, and M7' and M7" were separated by rechromatography (see Figs. 3 and 4). Peptides M5-DP and M3-4-5 which had some contaminating peptides were further purified using a Cosmosil $3 \mathrm{C} 18(0.46 \times 10 \mathrm{~cm})$ column (chromatograms not shown).

Fig. 3. Separation of M4-5 and M5 by HPLC. Peptides M4-5 and M5 found in one peak (Fig. 2) were further separated by HPLC. A TSK ODS-120T $(0.46 \times 25 \mathrm{~cm})$ column was used.

Fig. 4. Separation of $\mathrm{M}^{\prime}$ ' and M7" by HPLC. Peptides $\mathrm{M}^{\prime}$ and M7" found in one peak (Fig. 2) were separated by HPLC. A TSK ODS-120T $(0.46 \times 25 \mathrm{~cm})$ column was used.

Fig. 5. HPLC of subdigest of M1 with lysyl endopeptidase. A part of the digest was subjected to HPLC as described in 
and Methods. A TSK ODS-120T (0.46 x $15 \mathrm{~cm})$ column was used.

Fig. 6. HPLC of subdigest of MI with endoproteinase AspN. A part of the digest was subjected to HPLC as described in Materials and Methods. A Cosmosil 3C18 (0.46 × $10 \mathrm{~cm})$ column was used.

Fig. 7. HPLC of subdigest of M5 with lysyl endopeptidase. A part of the digest was subjected to HPLC as described in Materials and Methods. A TSK ODS-120T (0.46 x $15 \mathrm{~cm})$ column was used. Peptides M5K4 and M5K5 were separated by rechromatography (see Fig. 8).

Fig. 8. Separation of M5K4 and M5K5 by HPLC. Peptides M5K4 and M5K5 found in one peak (Fig. 7) were further separated by HPLC. A TSK ODS-120T (0.46 x $25 \mathrm{~cm})$ column was used.

Fig. 9. HPLC of subdigest of M5 with endoproteinase AspN. A part of the digest was subjected to HPLC as described in Materials and Methods. A Cosmosil 3C18 (0.46 x $10 \mathrm{~cm})$ column was used. Peptides M5D6 and M5D10, and M5D1, M5D7 and M5D12 were separated by rechromatography (see Figs. 10 and 11).

Fig. 10. Separation of M5D6 and M5D10 by HPLC. Peptides M5D6 and M5D10 found in one peak (Fig. 9) were further separated by HPLC. A TSK ODS-120T $(0.46 \times 25 \mathrm{~cm})$ column was used. 
Fig. 11. Separation of M5D1, M5D7 and M5D12 by HPLC. Peptides M5D1, M5D7 and M5D12 found in one peak (Fig. 9) were further separated by HPLC. A Cosmosil $3 \mathrm{C} 18(0.46 \times 10 \mathrm{~cm})$ column was used.

Fig. 12. HPLC of subdigest of M6 with lysyl endopeptidase. A part of the digest was subjected to HPLC as described in Materials and Methods. A TSK ODS-120T (0.46 x $15 \mathrm{~cm})$ column was used. Peptides M6K2 and M6K4 were separated by rechromatography (see Fig. 13).

Fig. 13. Separation of M6R2 and M6K4 by HPLC. Peptides M6K2 and M6K4 found in one peak (Fig. 12) were further separated by HPLC. A TSK ODS-120T $(0.46 \times 25 \mathrm{~cm})$ column was used.

Fig. 14. HPLC of subdigest with of M6 endoproteinase AspN. A part of the digest was subjected to HPLC as described in Materials and Methods. A Cosmosil $3 \mathrm{C} 18(0.46 \times 10 \mathrm{~cm})$ column was used. Peptides M6D2 and M6D7 were separated by rechromatography (see Fig. 15).

Fig. 15. Separation of M6D2 and M6D7 by HPLC. Peptides M6D2 and M6D7 found in one peak (Fig. 14) were further separated by HPLC. A TSK ODS-120T $(0.46 \times 25 \mathrm{~cm})$ column was used.

\section{Fig. 16. HPLC of tryptic peptides derived from} succinylated Pe-HR1B. A part of the digest was subjected to HPLC as described in Materials and Methods. A Cosmosil 5C4 (0.46 x $15 \mathrm{~cm}$ ) column was used. ST4-5 and ST5 were contaminated and 
using a Cosmosil $3 \mathrm{C} 18(0.46 \times 10 \mathrm{~cm})$ column (chromatograms not shown).

Fig. 17. HPLC of digest of Pe-HR1B with arginylendopeptidase. A part of the digest was subjected to HPLC as described in Materials and Methods. A TSK ODS-120T (0.46 x $15 \mathrm{~cm}$ ) column was used.

Fig. 18. Structural relationships of HR1B with other proteins. A, Sequence comparison of the amino-terminal region of HR1B with HR2a, Ht-d, and H2-proteinase. HR2a (7), Ht-d (6), and H2-proteinase (27) are low molecular mass metalloproteinases. $H R 1 B, H R 2 a$, and $\mathrm{H}_{2}$-proteinase were isolated from the venom of $T$. flavoviridis, and Ht-d from C. atrox. Identical residues shared by all proteins are shaded. The residues shared by hemorrhagic metalloproteinases, HR1B, HR2a, and Ht-d and not shared by nonhemorrhagic $\mathrm{H}_{2}$-proteinase are boxed. The putative zinc ligands and active site are indicated by $\boldsymbol{\Lambda}$ and $\Delta$, respectively.

indicate sugar chains linked to Asn residues of HR1B. <E denotes 5-pyrrolidone-2-carboxylic acid. B, Comparison and alignment of the carboxyl-terminal portion of HR1B with several disintegrins. Trigramin (17), Echistatin (29), Applaggin (31), and Bitistatin (32) are all viper venom platelet aggregation inhibitors, called disintegrins (35). Cysteine residues are boxed. The Arg-GIy-Asp-sequence common to disintegrins are shaded.

Fig. 19. Comparison and alignment of the sequences of HR1B and human von Willebrand factor (VWF). Identical residues are boxed. 

20

40

50

60

70

80

90

100

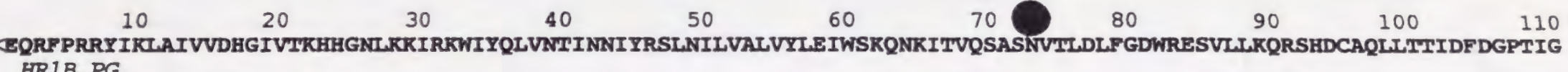
HR1B PG

QRFPRRYIKLAIVVDHGIVTKHH

\begin{tabular}{|c|c|}
\hline \multicolumn{2}{|c|}{$\begin{array}{l}\text { M1K2 } \\
\text { IAIVVDHGIVTK }\end{array}$} \\
\hline & \\
\hline$M I K I \quad P G$ & M1K3 \\
\hline RFPRRYIK & HHGNLK \\
\hline
\end{tabular}

$M 1 K 4$

WIYQLVNTINNI YRSLNILVALVYLEIWSK

DHGIVTKHHGNLKKRIRKWI YQLVNTINNI YRSLNILVALVYLEI-SKQNRITV

$$
\text { MIN1 }
$$

M1K5

M1R6

QRSHDCAQLLTTIDFDGPTIG

ITVQSAS-VTLDLFG-W-E-VLL DCAQLLTTI

SKONRITVQSAS-VTLDLFGD SHDCAQLLTTIDFDGPTIG
120
130.140
150
160
170190
200

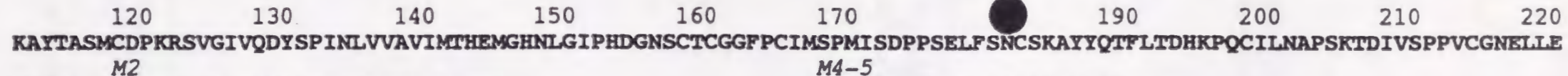

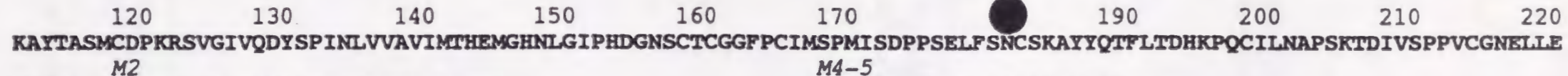

(M1K6) $\stackrel{\text { M2 }}{\text { CDPKRSVGIVQDYSPINLVVAVIMTHEM }}$ $\mathrm{K}$ AYTAS

(M1D6)

(MID6)

(ST2) M3
GHNLGIPHDGNSCTCGGFPCIM

SPMISDPPSELFS-CSKAYYQTFI M5-DP $M 5$ PPSELFS-CSKAYYQTFLTDHKPQCILNAPSKTDIVSPPVCGNELI

-AYTASMCDP RI SVGIVQDYSPINLVVAVIMTHEMGHNLGIPH ISDPPSELFS-CSKAYYQTFLTDHKPQCILNAPSKTDIVSPPVCG

$$
\text { M3-4-5 }
$$
M5K1

$\begin{array}{lll} & \text { M5K1 } & \text { M5K3 } \\ \text { M5D1 } & \text { AYYQTFLTDHK } & \text { TDIVSPPVCGNELLE }\end{array}$
M5D1 M5D3

ELFSNCSKAYY DHKPOCILNAPSKT

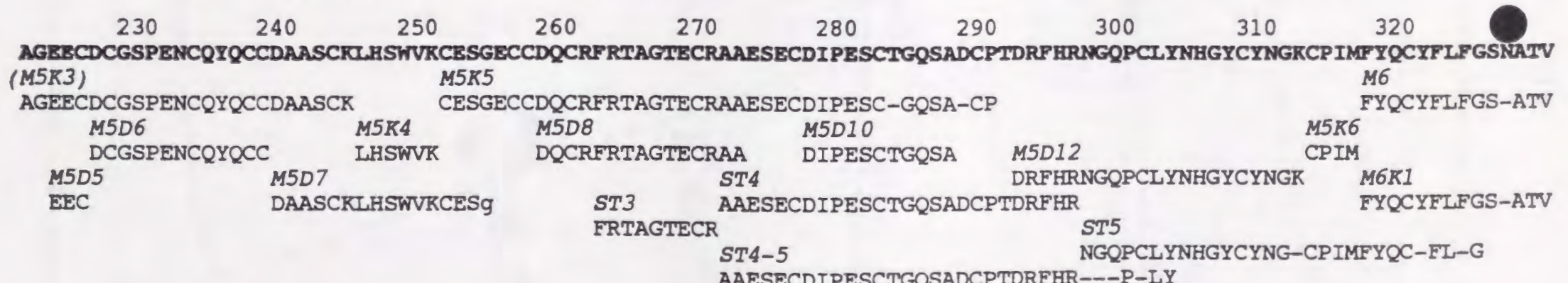

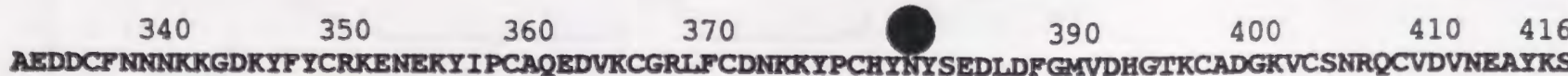

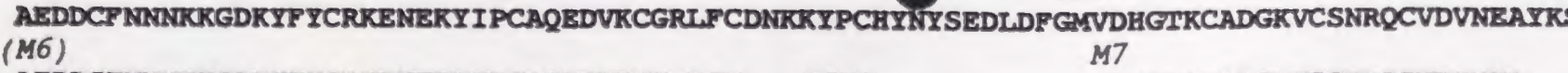

$\begin{array}{ccl}\text { AEDDCFNMNKRGDKYFYCRKENEKYIPCAQEDVKCG-LFC } & \text { M6R5 } \\ \text { M6R2 } & \text { M6K4 } & \text { KYPCHYNYSEDLDFGM }\end{array}$

(M6R1) MFR2 MFR M6R3 CGRLFCDNK KYPCHYNYSEDLDFGM M7'

YIPCAQEDUK MVDG

M6D2

DNKKYPCHYกYSE MT"

NCADGKVCSNRQCVDVNEAYR

LFCDN--YPCHY-YSEDLDFGMVDHGT-CADG-VCSNR

Fig. 1 


\section{(--) \% 3า14LINOL3ว}

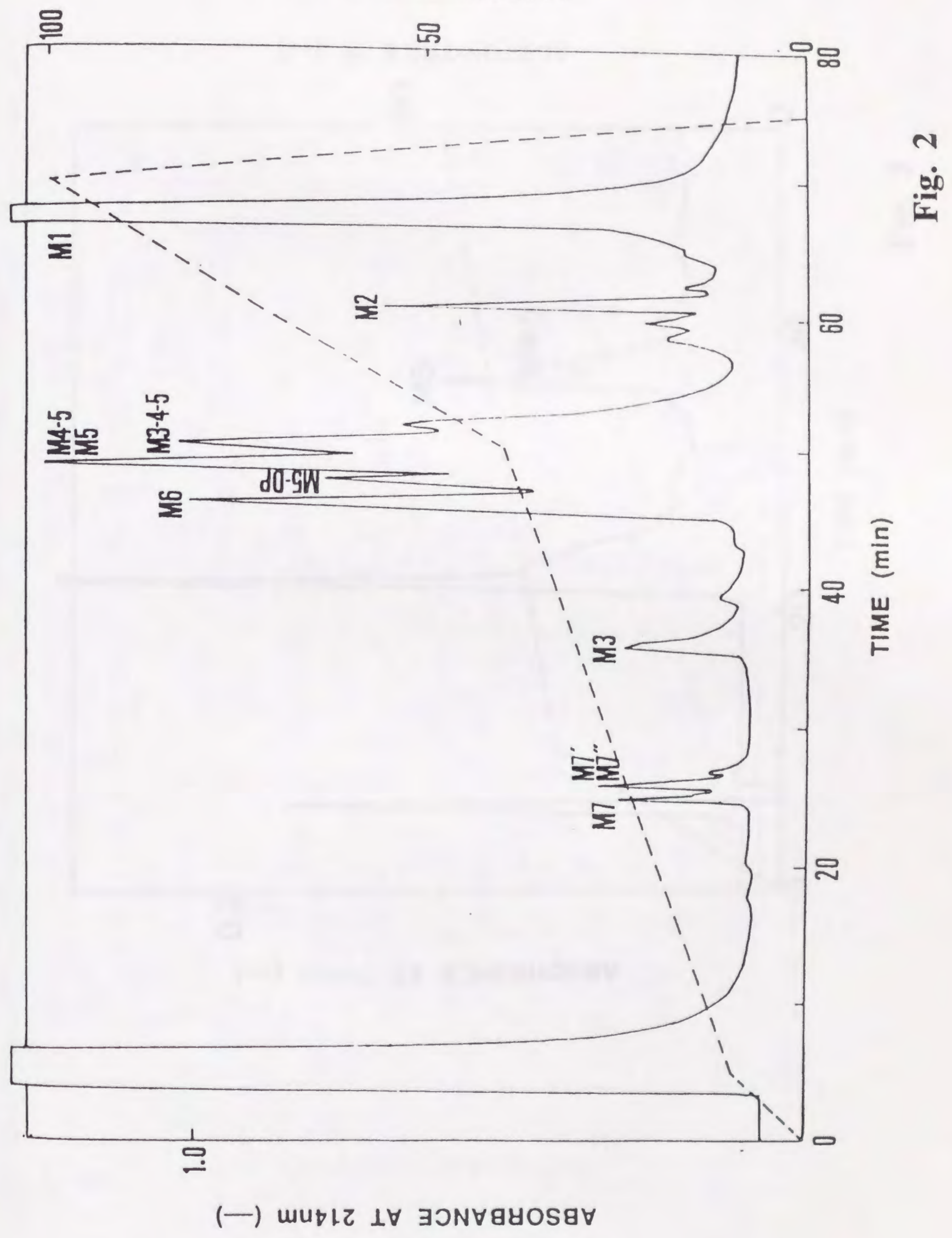




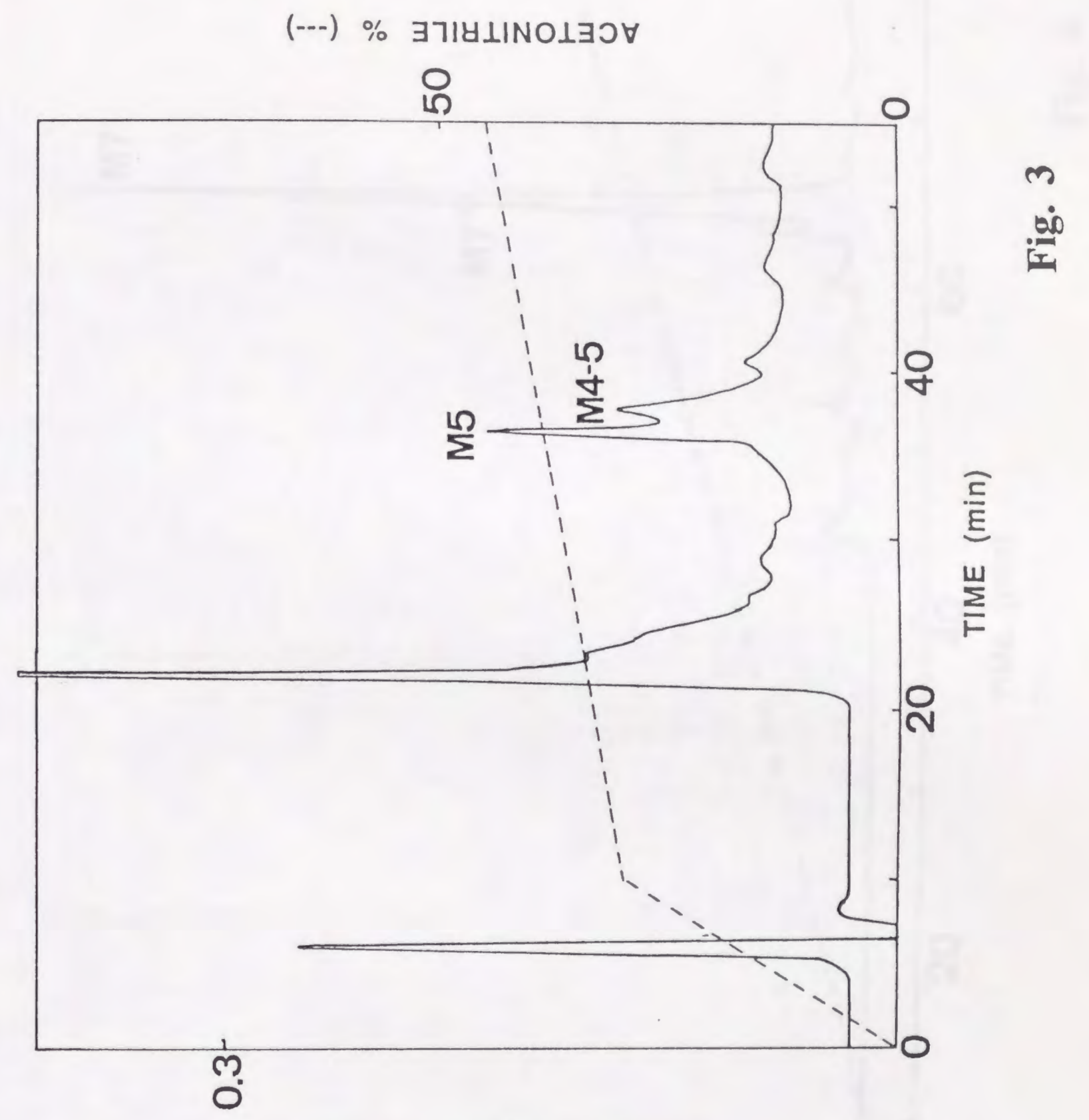

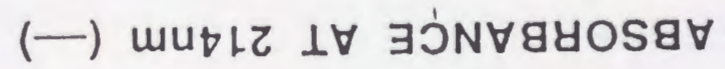




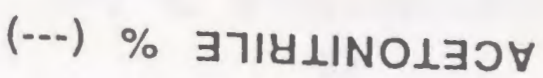

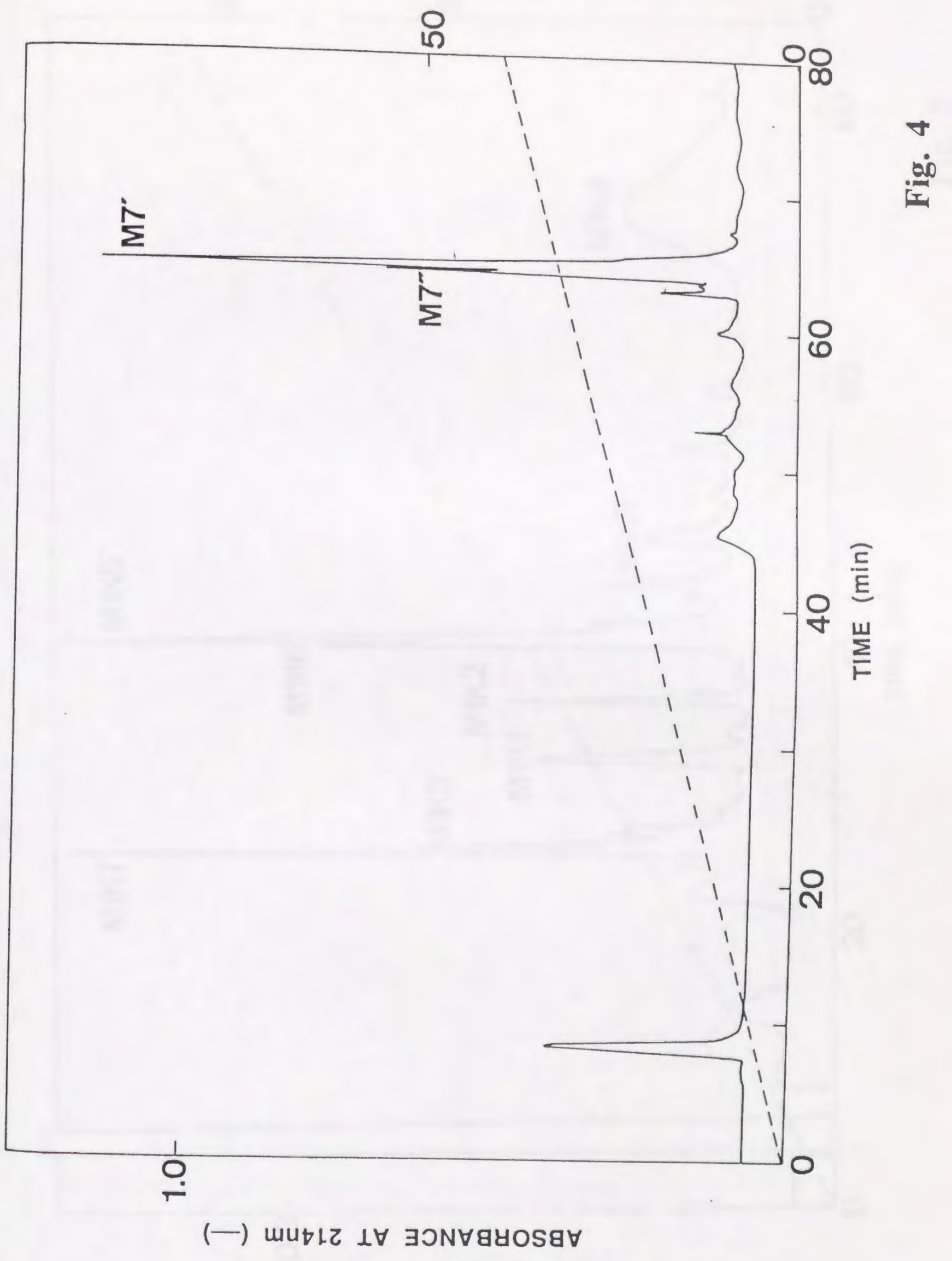




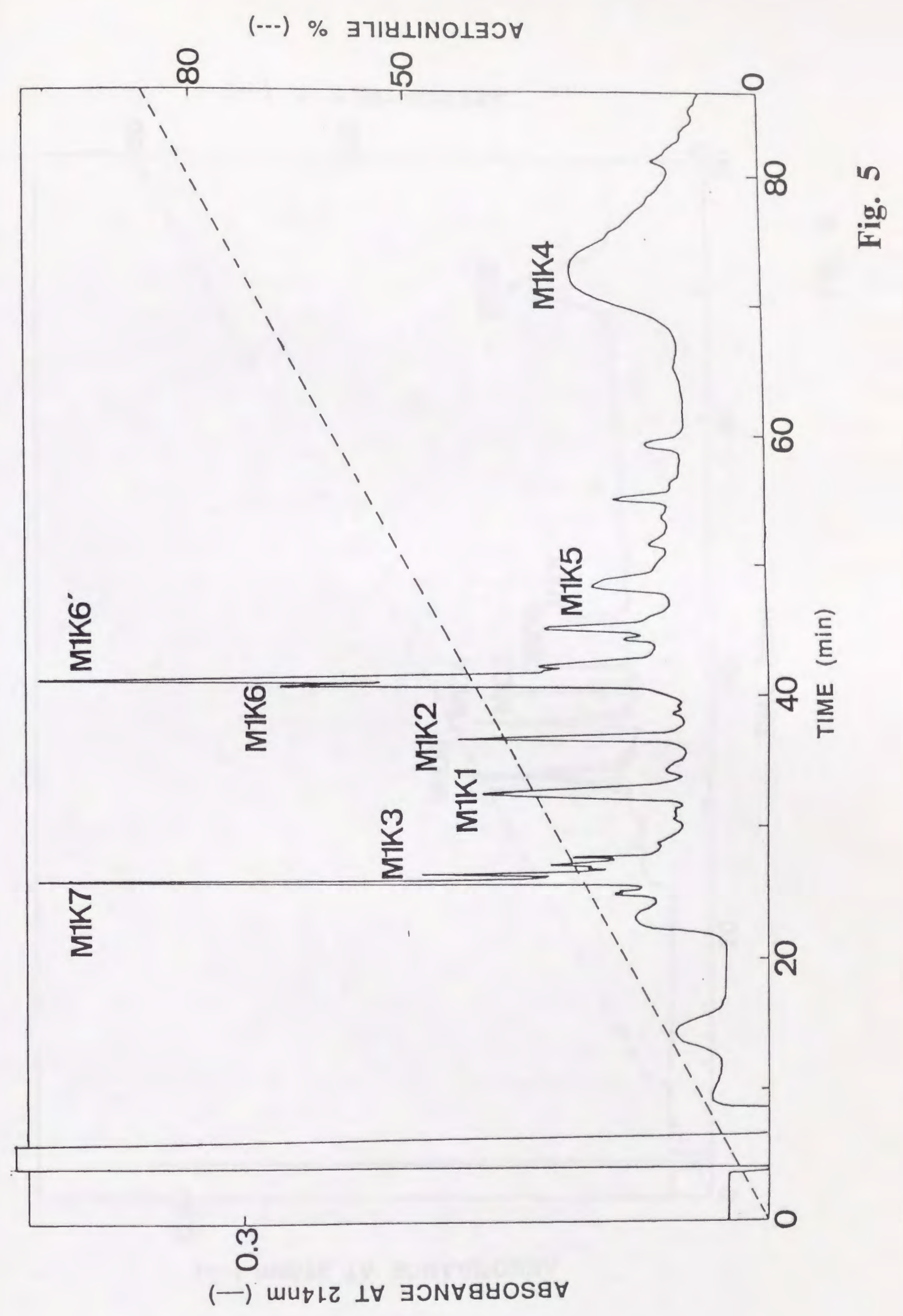




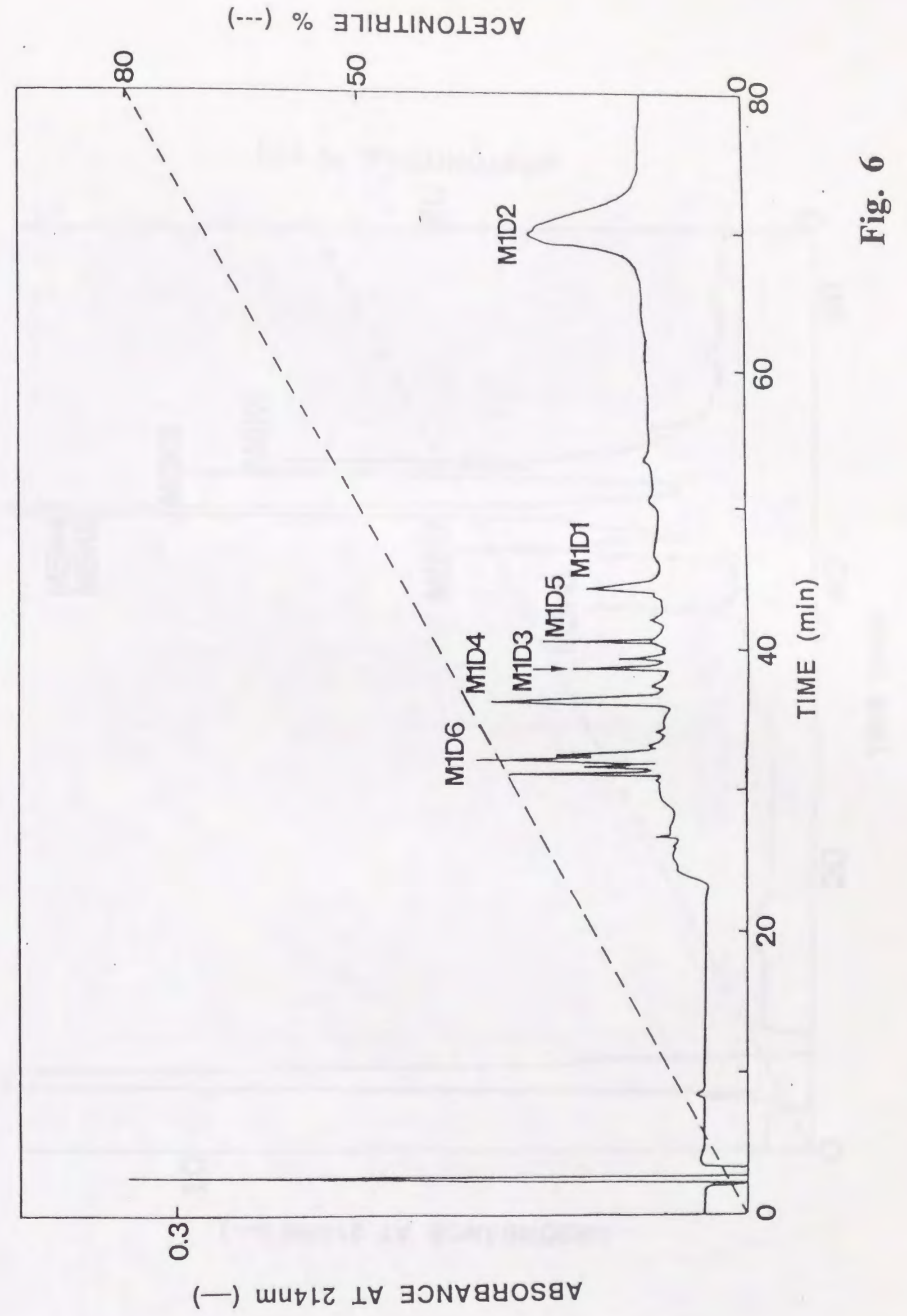




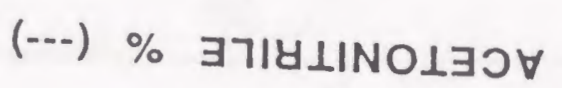

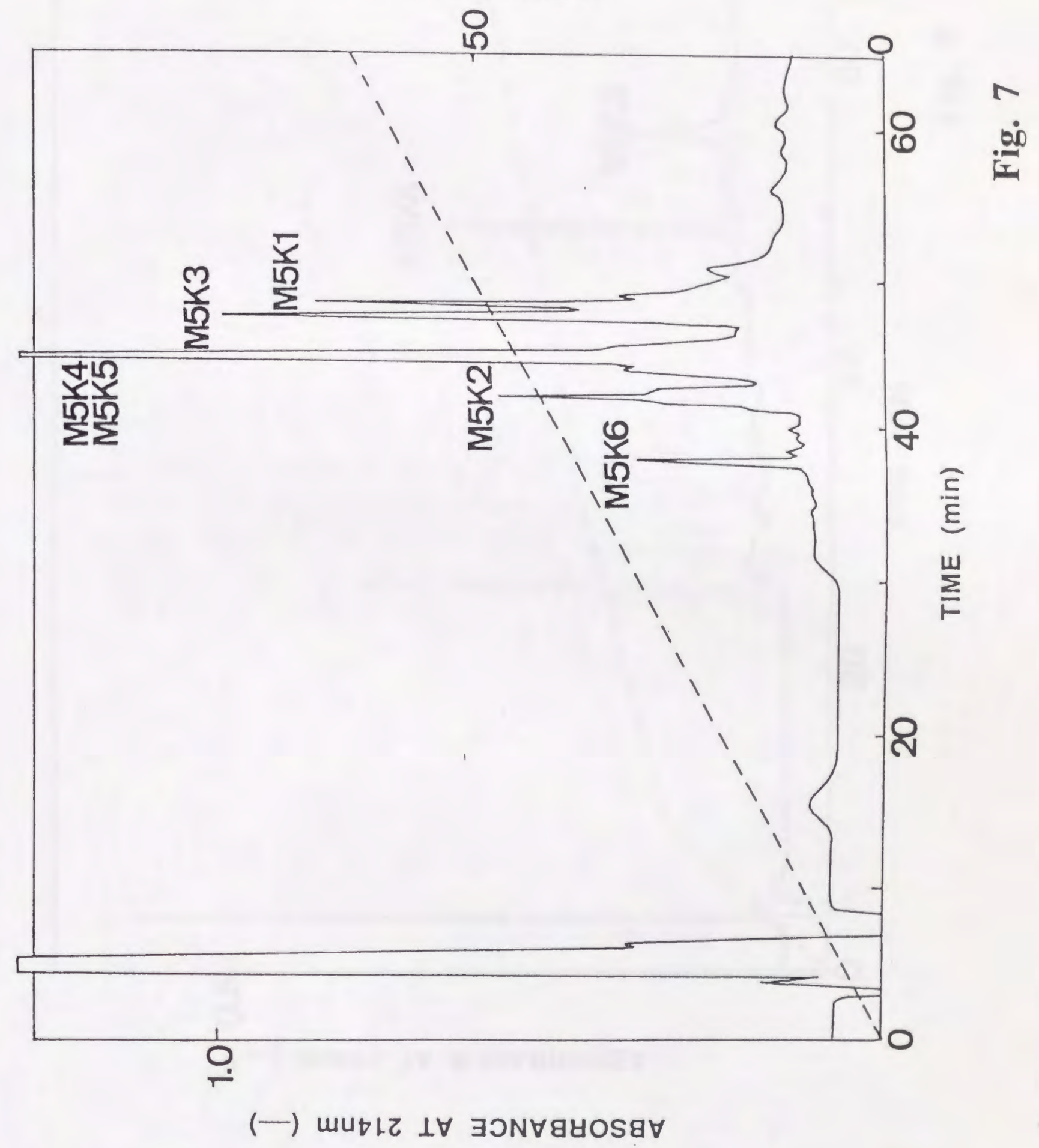




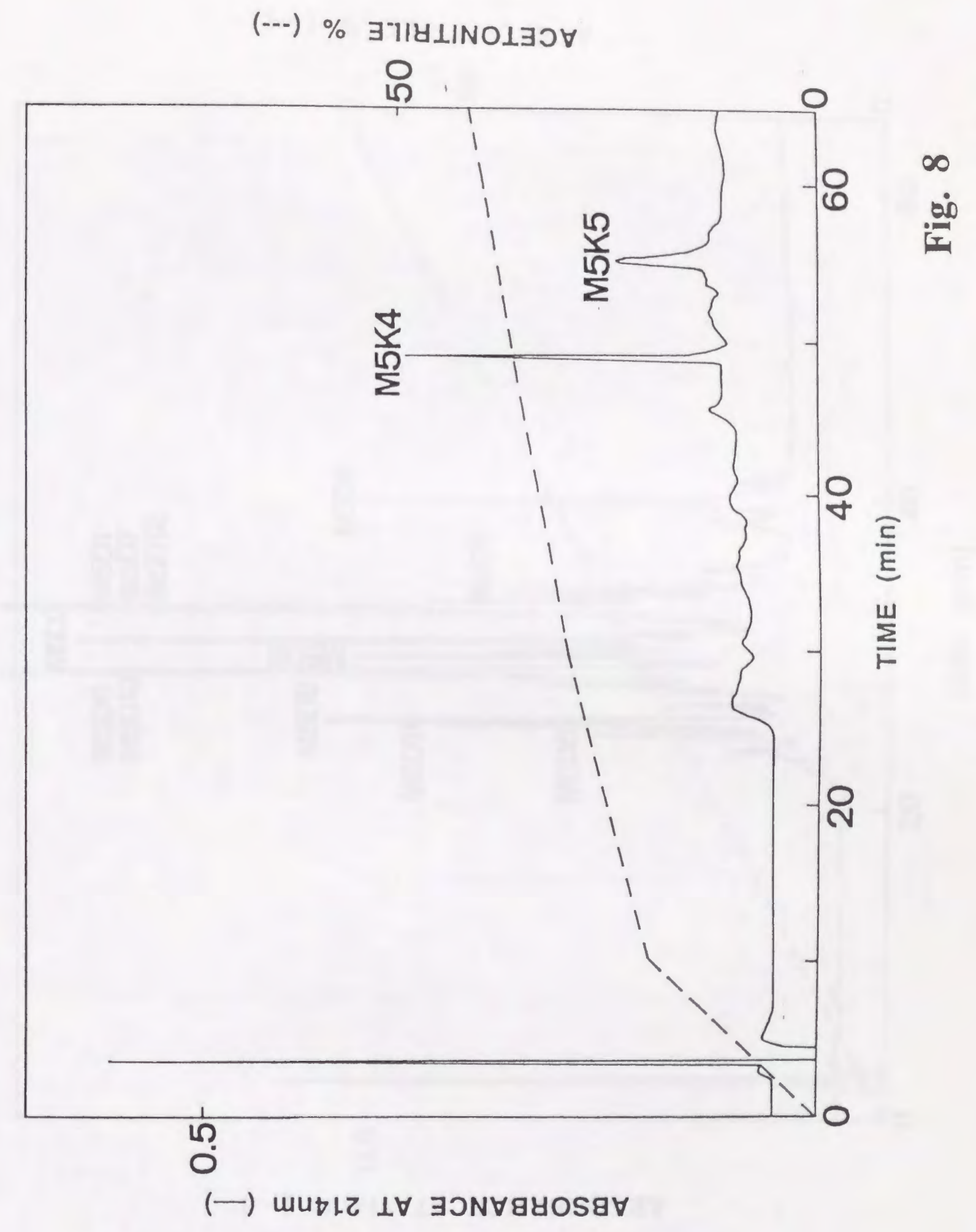




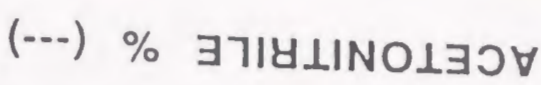

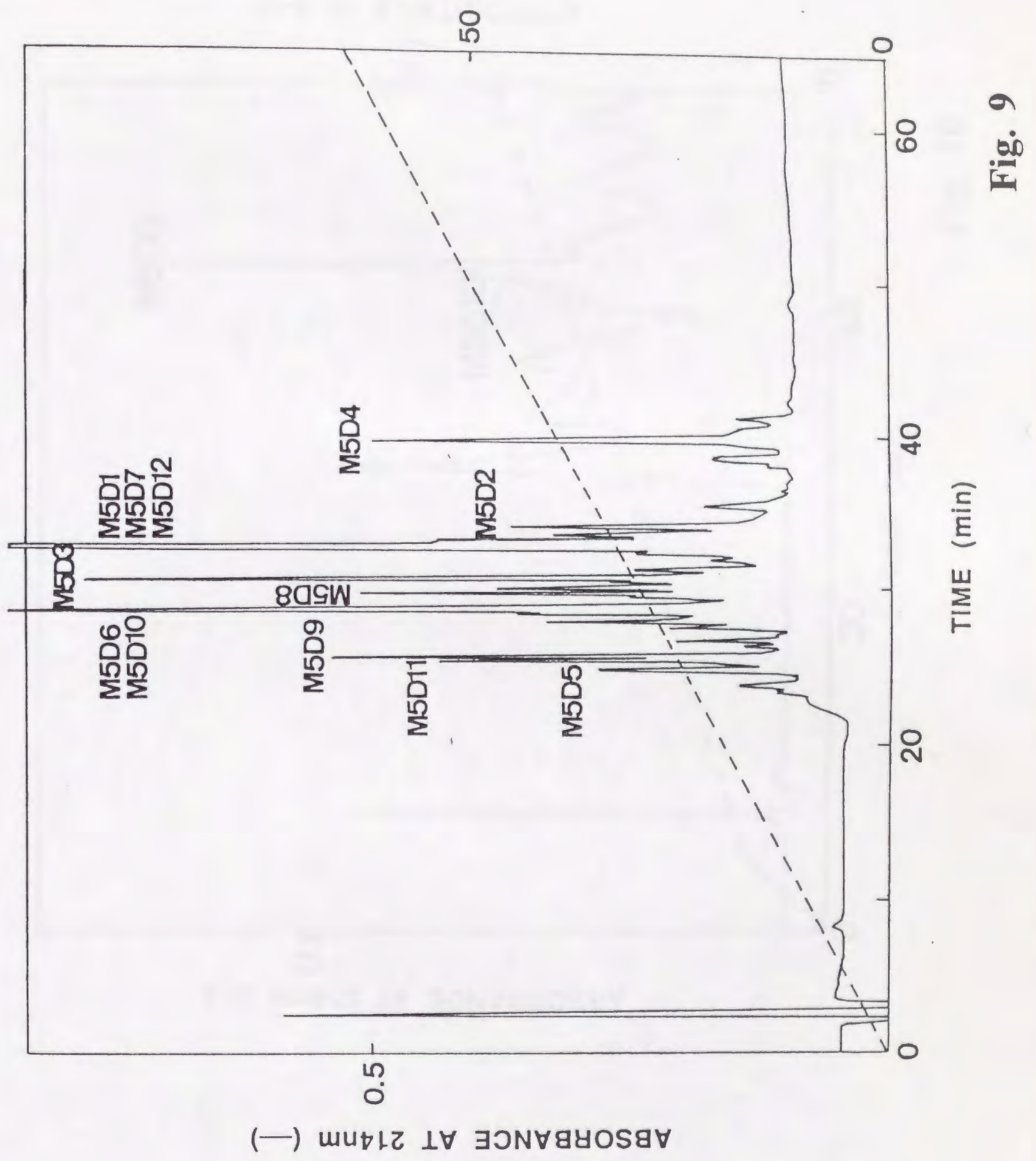


(---) \% ヨา

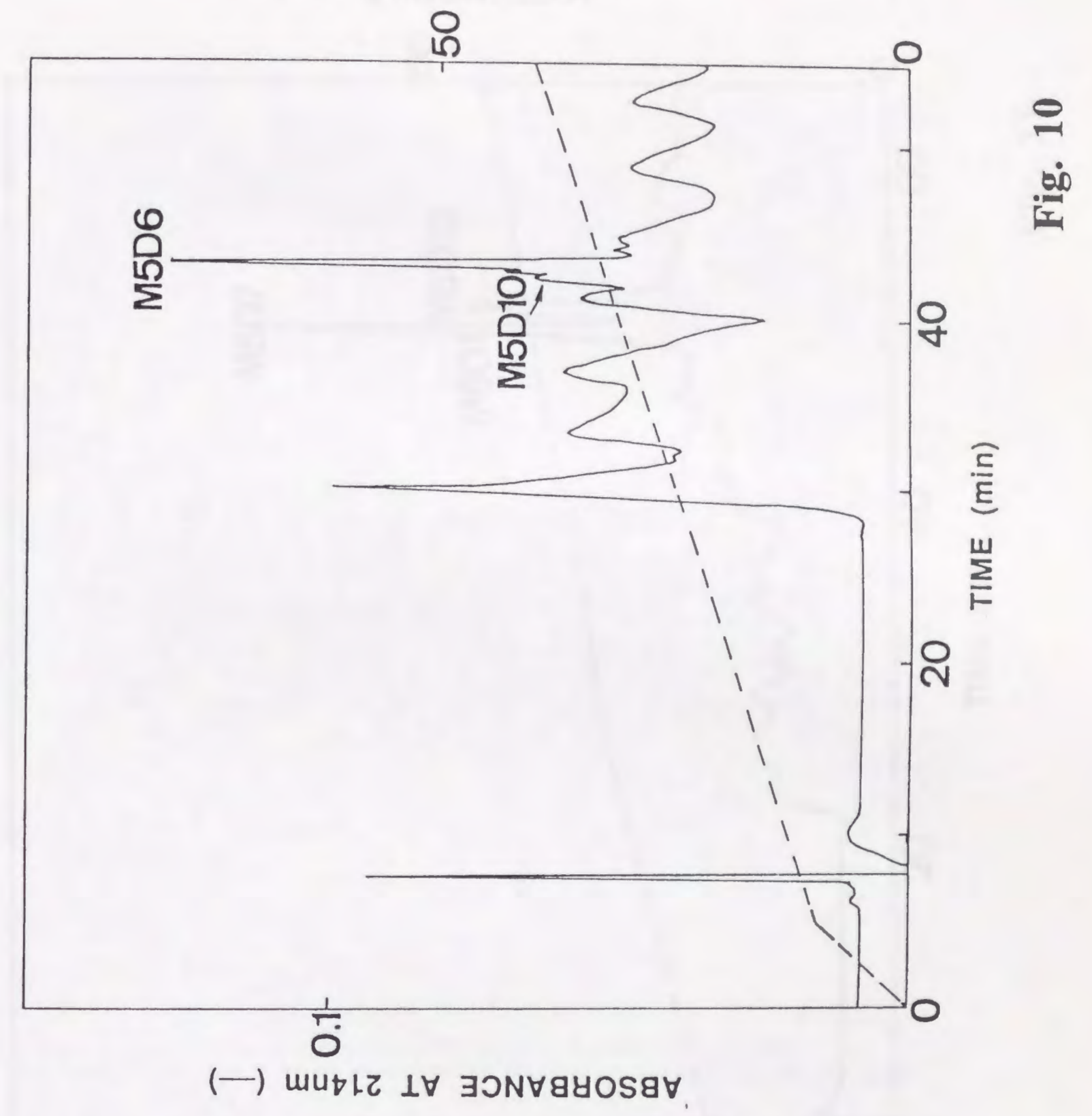




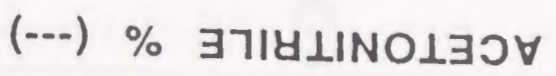

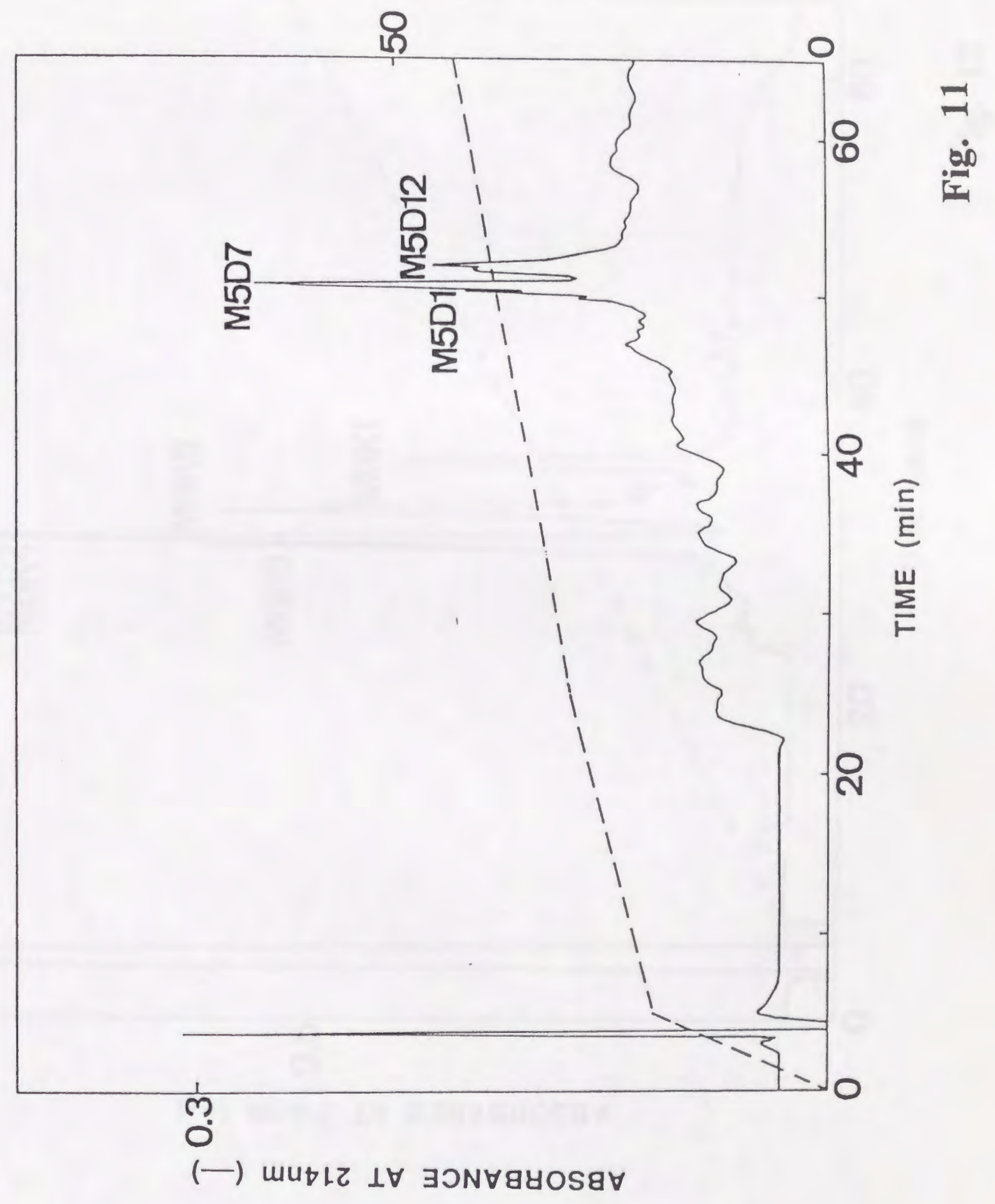




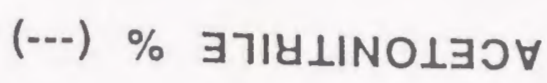

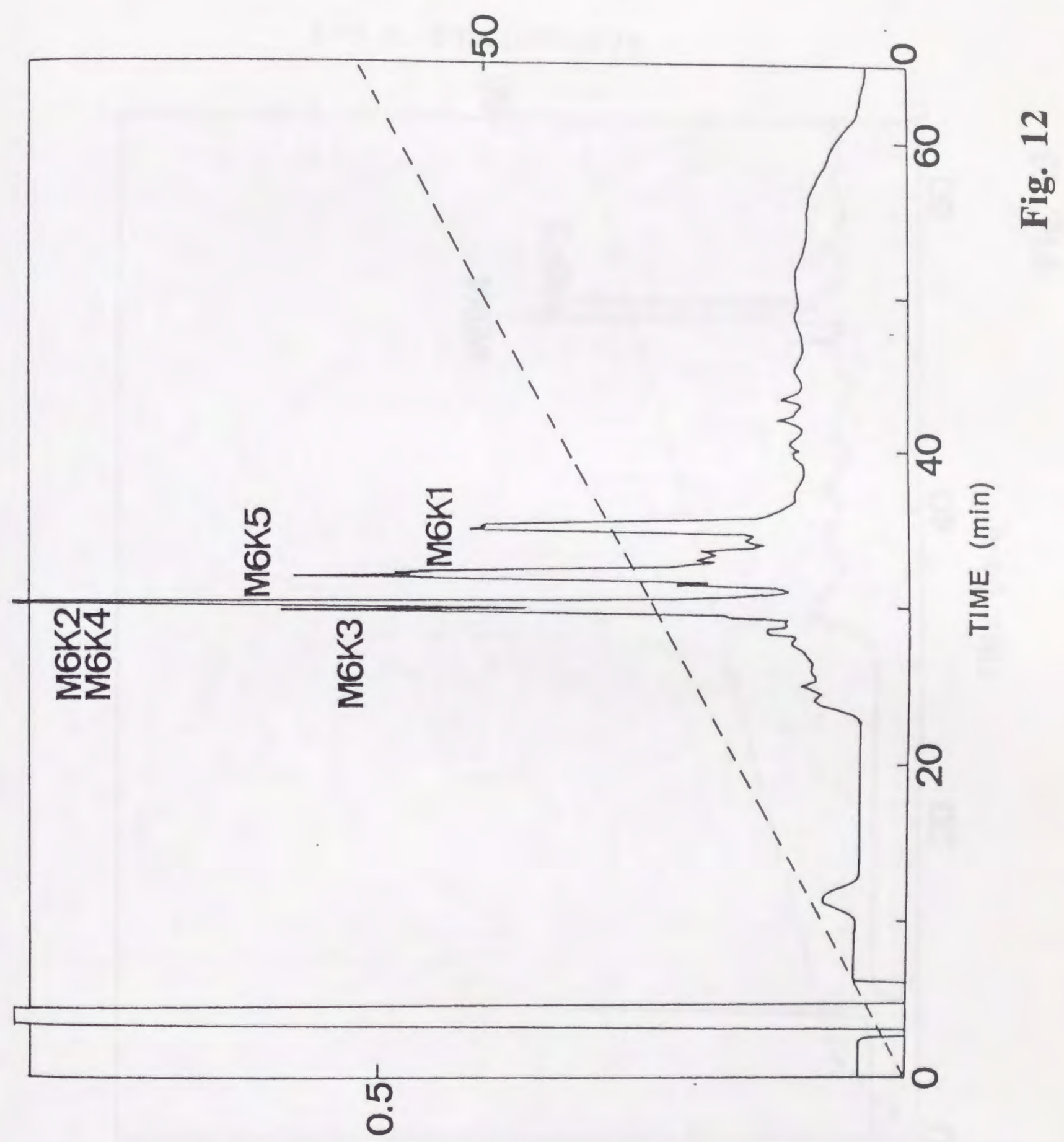

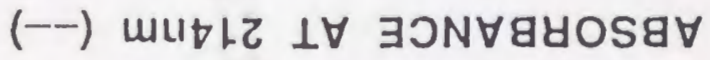




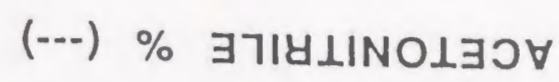

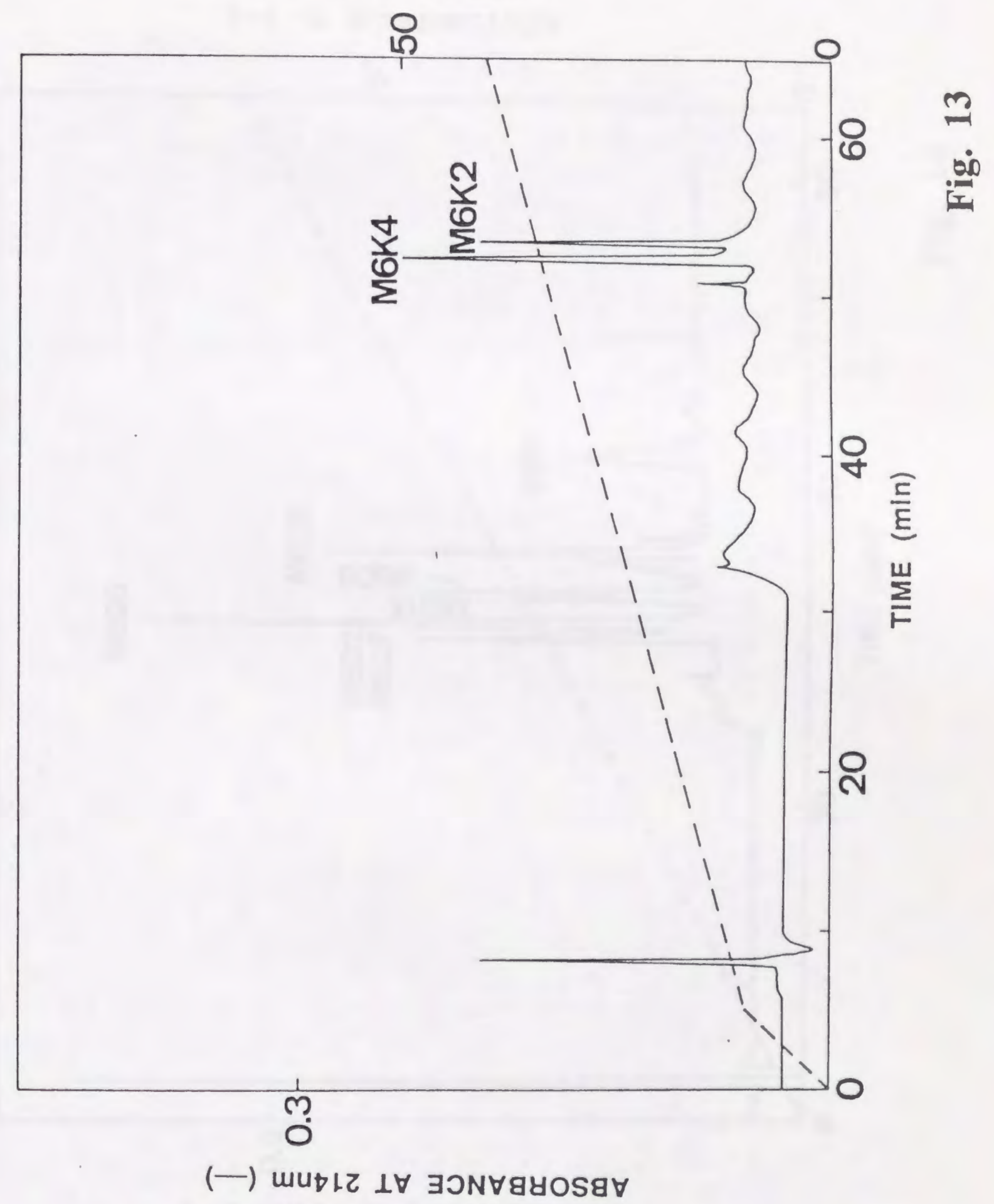




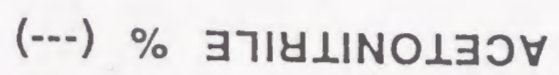

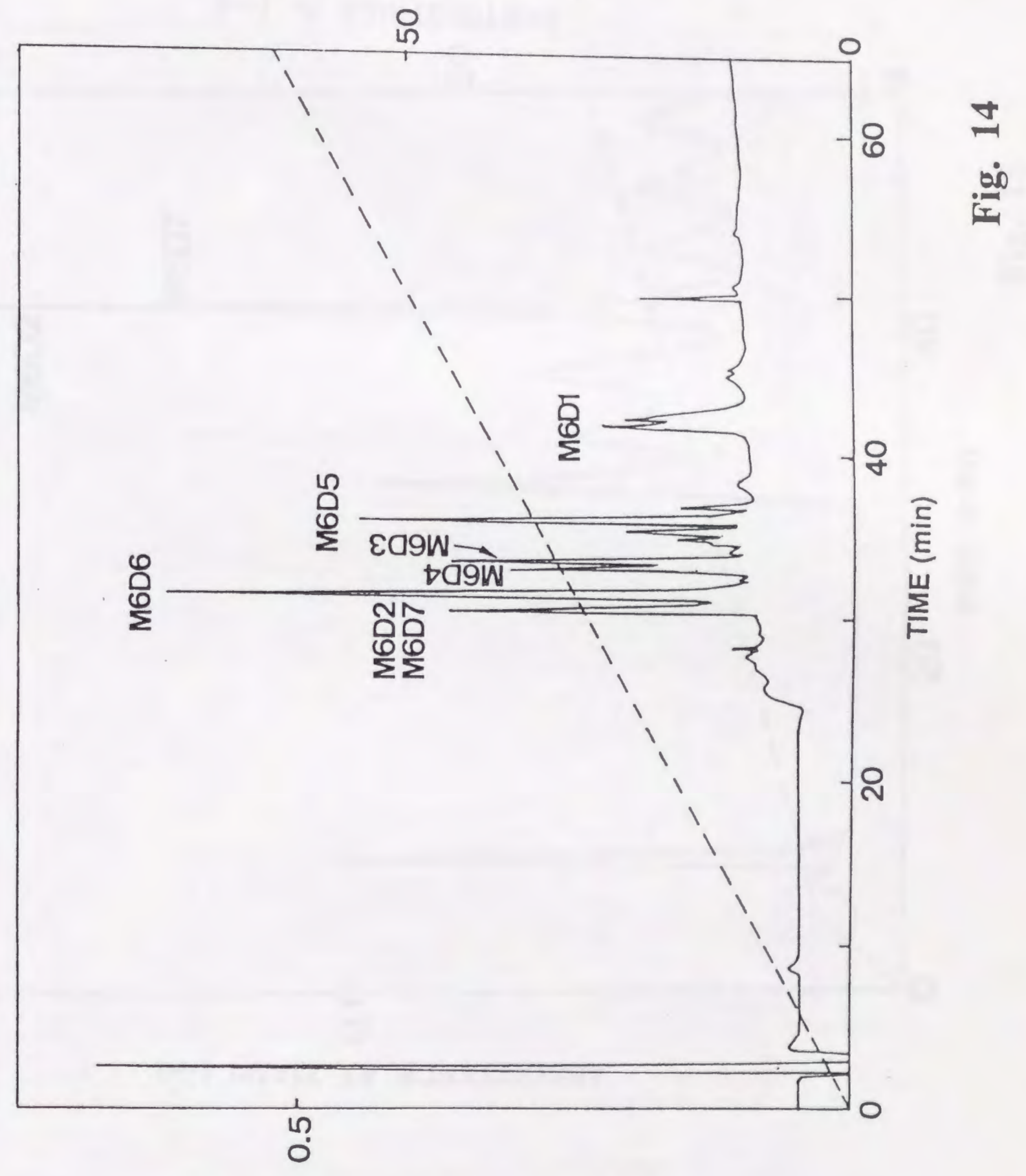

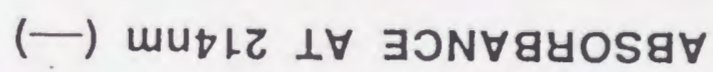




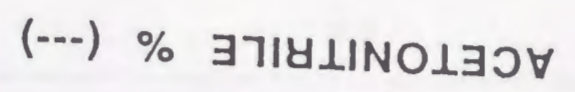

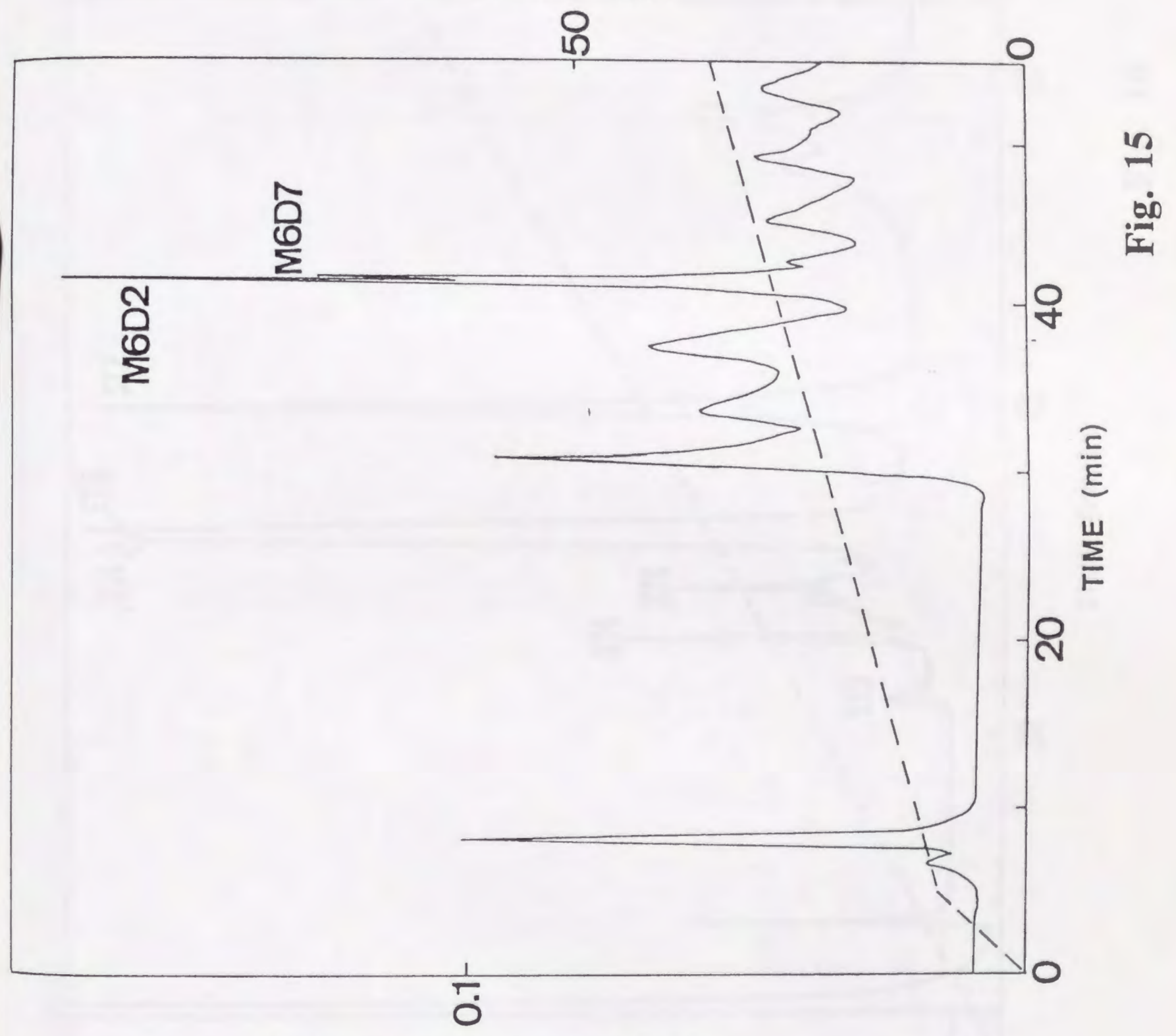

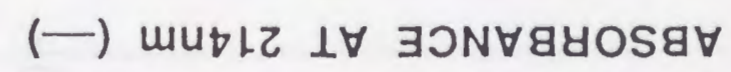


(---) \% ヨา

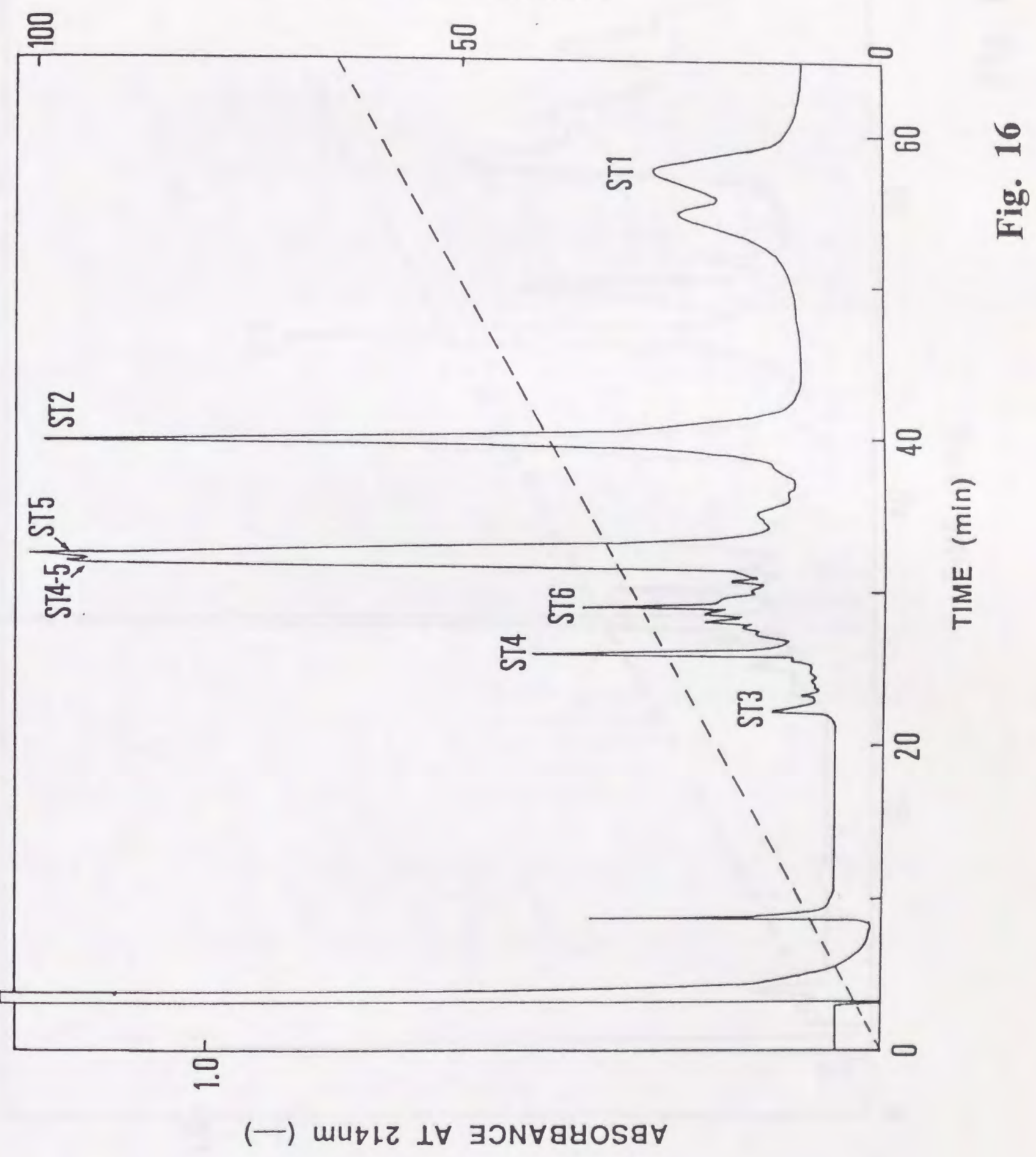




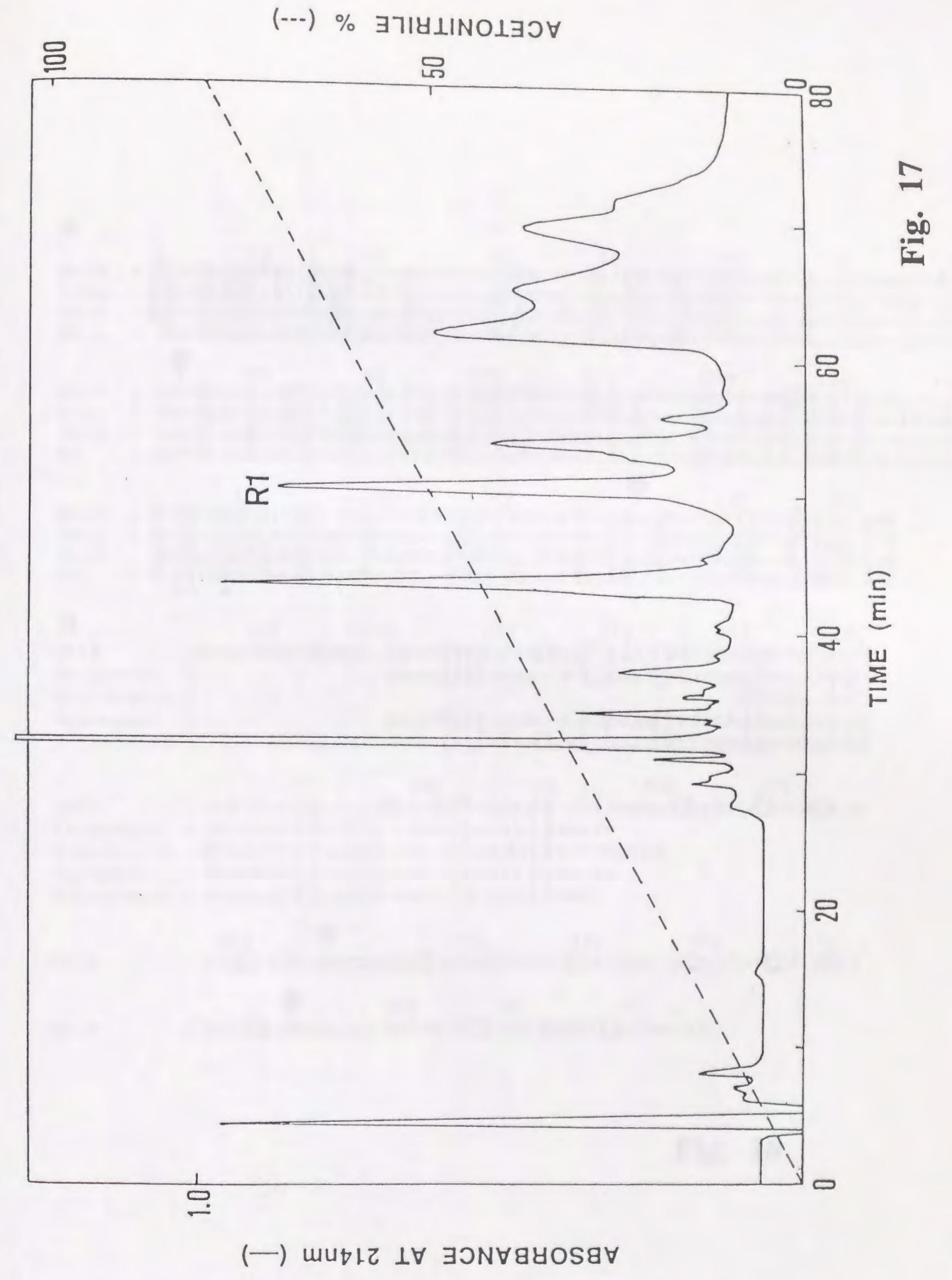


A

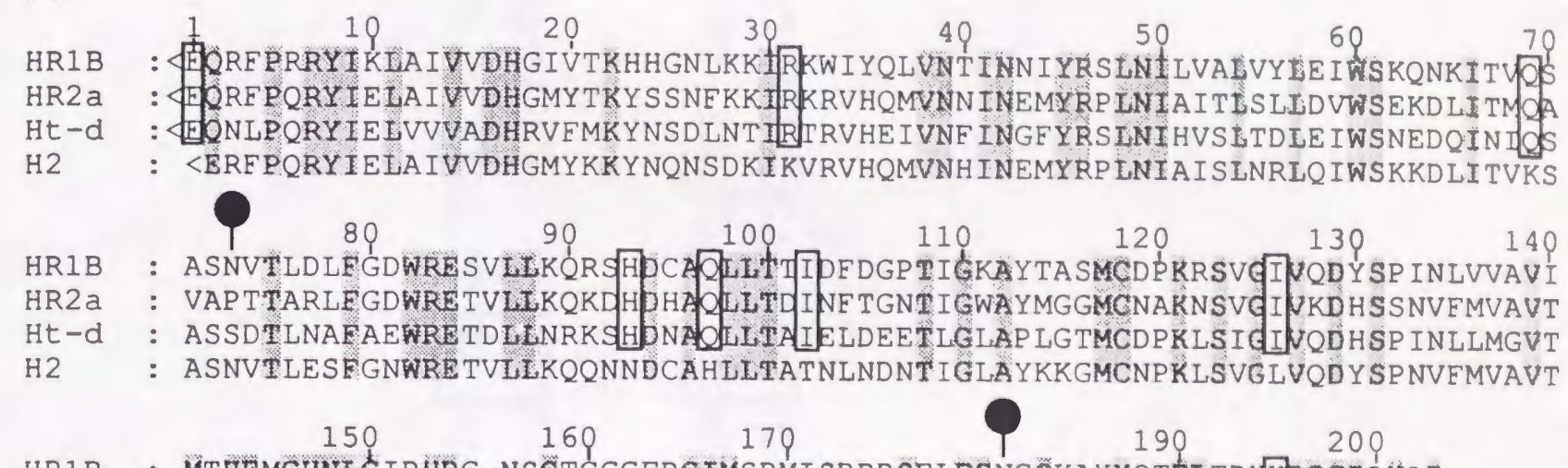

HR1B : MTHEMGHNLG IPHDG-NSC̈TCGGFPCIMSPMI SDPPSELESNCSKAYYQTETTDHKPOCILNAP

HR2a : MTHE I GHNLGME HDDKDKCKCEA--CIMSAVI SDKP SKIF SDC SKDYYOTFETNSKQOCI INAP

Ht-d : MAHELGHNLGMEHDGKD-CLRGASLCIMRP GLTKGRSYEFSDD SMHYYERFLKQYKPOCI LNKP

H2 : MTHE LGHNLGMEHD DKDKCKCEA--CIMSDVISDKP SKLFSDCSKNDYOTELTKYNPOCILNAP

$\sim \mathrm{A}$

\section{B}

HRIB

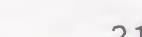

$210 \quad 22$

$230 \quad 240 \quad 260$

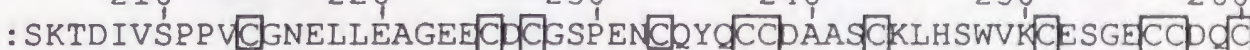
EAGEDCDCGSPAN--P-CCDAATCKL IP GA QGGEGICCDOC EAGEECDCGSPEN--P-CCDAATCKLRPGAOCAEGICCDOC Echistatin : Applaggin : Bitistatin : SPP VCGNE I LEQGEDCDCGSPANCDD CCCNAA ICKLTP GS dCNHGEC D dC

HR1B : RFRTAGTECRAAESECDIPESCTGQSADCPTDRFHRNGQṔCL YNHGYCYNGKCP IM

Trigramin : SFIEEGTYCRIAR̈i-DLDDYCNGRSACCPRNPFH

Echistat in : KFLKEGTICKRA_GL-DMDDICNGKTCDCPRNP HKGPAT

Applaggin : KFMKEGTUCR-A H. W-DVNDYCNGISACCPRNPFH

Bitistatin : KEKKARTUCRIAH

HR1B : FYdCYFLFGSNATVAEDDCFNNNKKGDKYFYCRKENEKYIPCAQEDVACGRLECDN

HR1B : KKYPCHYNYSEDLDFGMVDHGTKCADGKUCSNROCVDUNEAYKS

\author{
Fig. 18
}




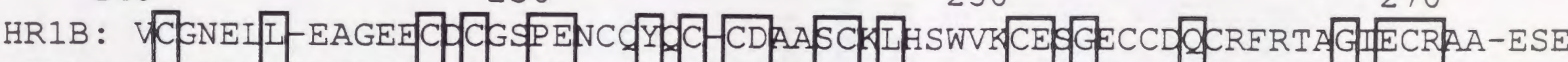

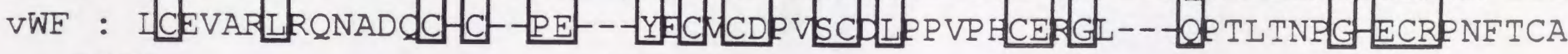
1543

$$
1560
$$$$
1580
$$

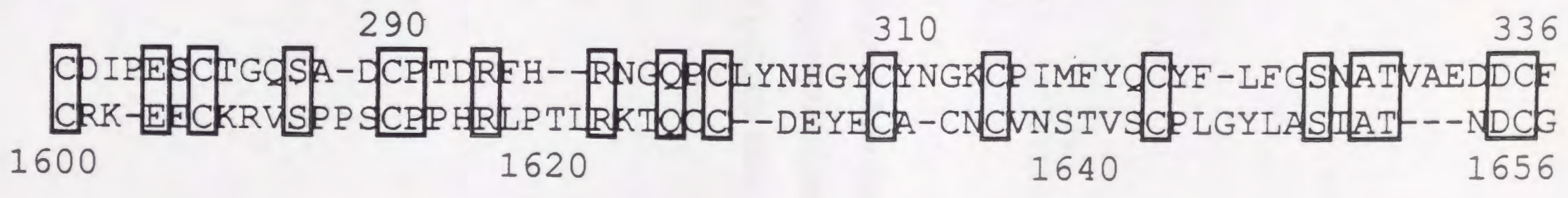

Fig. 19 
PART II

Coagulation Factor X Activating Enzyme from Russell's Viper VenOm $(R V V-X)$ : A NOVEL METALLOPROTEINASE WITH DISINTEGRIN (PLATELET AGGREGATION INHIBITOR)-LIKE AND C-TYPE LECTIN-LIKE DOMAINS 


\section{SUMMAR Y}

We determined the complete amino acid sequence of RVV-X, the blood coagulation factor $x$ activating enzyme, isolated from Russell's viper venom and studied the structure-function relationship. RVV-X (Mr 79,000) consisted of a disulfide-bonded two-chain glycoprotein with a heavy chain of Mr 59,000 and a light chain of heterogeneous $\operatorname{Mr} 18,000$ or 21,000 . These chains were separated by gel filtration after reduction and $S-$ pyridylethylation. Two heterogeneous light chains, designated LC1 ( Mr 18,000) and LC2 (Mr 21,000), were separated by reversed phase high performance liquid chromatography, and the isolated major component LCl was used for sequence analysis. The heavy chain consisted of 427 residues containing four asparagine-linked oligosaccharides, and its entire sequence was similar to that of the high molecular mass hemorrhagic protein, HRIB, isolated from the venom of Trimeresurus flavoviridis (Takeya, H., Oda, K., Miyata, T., Omori-Satoh, T., and Iwanaga, S. (1990) J. Biol. Chem. 265, 16068-16073). The heavy chain contained three distinct domains, metalloproteinase, disintegrin (platelet aggregation inhibitor)-like and unknown cysteine-rich domains. On the other hand, the light chain LC1 consisted of 123 amino acid residues containing one asparagine-linked oligosaccharide and showed a sequence homology similar to those found in the so-called c-type ( $\mathrm{Ca}^{2+}$-dependent) lectin. Therefore, RVV-X is a novel metalloproteinase containing a mosaic structure with disintegrinlike, cysteine-rich, and C-type lectin-like domains. RVV-X potently inhibited collagen- and ADP-stimulated platelet aggregations, probably via its disintegrin-like domain, although 
this domain does not contain the Arg-Gly-Asp sequence which is conserved in various venom disintegrins and which is thought to be one of the interaction sites for platelet integrins. Our findings also indicate that snake venom factor IX/factor $\mathrm{X}$-binding protein with a C-type lectin structure (Atoda, H., Hyuga, M., and Morita, T. (1991) J. Biol. Chem. 266, 14903-14911) inhibits the RVV-Xcatalyzed factor $\mathrm{x}$ activation, hence, the light chain of RVV-X probably participates in recognizing some portion of the zymogen factor $\mathrm{x}$. 


\section{INTRODUCTION}

Snake venoms have various proteins and enzymes which affect mammalian blood coagulation and fibrinolytic systems (1). These components have a strict specificity in their contacts with blood coagulation factors (2-5). Russell's viper (Vipera russelli) venom contains two well known protea'ses, designated RVV-V and RVV$\mathrm{x}$, both of which induce coagulation of mammalian plasma. We reported earlier that $R V V-V$, which specifically activates factor $V$ by limited proteolysis, consists of 236 amino acid residues and shows a sequence homology similar to that of trypsin-like serine proteinases, especially batroxobin, a thrombin-like enzyme isolated from the venom of Bothrops atrox (6).

$\mathrm{RVV}-\mathrm{X}$, a potent activator of factor $\mathrm{X}$, is a well

characterized proteinase (7) which activates specifically factor $\mathrm{X}$ as a result of a single cleavage at the same internal Arg-Ile bond in factor $X$ as do factors IXa and VIIa $(8,9)$. The RVV-X-catalyzed factor $\mathrm{X}$ activation, however, is not inhibited by disopropyl fluorophosphate and phenylmethylsulfonyl fluoride but is inhibited by EDTA, thereby suggesting that RVV-X is not a serine proteinase but a metalloproteinase $(10,11)$. Indeed, RVV-X contains nonexchangeable $1 \mathrm{~mol}$ of $\mathrm{Ca}^{2+}$ and $0.7 \mathrm{~mol}$ of $\mathrm{Zn}^{2+}$ essential for the proteolytic activity (12). Unlike vitamin $\mathrm{K}$-dependent clotting factors, RVV-X does not require a negatively charged surface such as phospholipids for factor $\mathrm{X}$ activation, but it does require exogenous $\mathrm{Ca}^{2+}$ and the amino-terminal Gla-domain of factor $\mathrm{X}$ for enhanced activation (13). This reversibly bound $\mathrm{Ca}^{2+}$ is not essential for the proteolytic activity of RVV-X, since RVV-X is 
able to hydrolyze apoprotein AI of human high-density lipoprotein, even in the absence of exogenous $\mathrm{Ca}^{2+}(12)$. RVV-X is a glycoprotein containing $13 \%$ carbohydrate with an apparent Mr of 79,000 . It is composed of two disulfide bonded chains, a heavy chain of Mr 59,000 and heterogeneous light chains with $\mathrm{Mr} 18,000$ and 21,000. In the present study, we determined the entire amino acid sequence of $\mathrm{RVV}-\mathrm{X}$, in order to elucidate the molecular mechanism of the RVV-X-catalyzed factor X activation, in particular how RVV-X specifically recognizes factor $\mathrm{X}$. Our interest in the structure of $\mathrm{RVV}-\mathrm{X}$ is because it is a unique metalloproteinase with respect to strict substrate specificity (7). Since we identified the entire amino acid sequences of several hemorrhagic and non-hemorrhagic metalloproteinases isolated from snake venoms and as these are new members of the metalloproteinase subfamily (14-17), it was also of interest to compare the covalent structures between RVV-X and the venom metalloproteinases. 


\section{MATERIALS AND METHODS}

Materials The sources of materials used were as follows: The venom of Vipera russelli siamensis from the Japan Snake Institute, Gunma; Achromobacter lyticus lysyl endopeptidase, and 4-vinylpyridine from wako Pure Chemical Industries, Osaka; endoproteinase Asp-N from Boehringer-Mannheim Biochemica, Mannheim; trypsin treated with $N$-tosyl-phenylalanyl chloromethyl ketone and $\alpha$-chymotrypsin from worthington Biochemical, Freehold, N. J.; Staphylococcus aureus v8 protease from Miles Laboratories, Elkhart, Ind.; cyanogen bromide, and Cosmosil 5C4-300 column from Nacalai Tesque, Inc., Kyoto; Phenyl5pw RP, G3000SW and G2000SW columns from Tosoh, Osaka; Chemcosorb 5-ODS-H column from Chemco Scientific Co., Ltd., Osaka; Vydac 214 TP5415 column from The Separations Group, CA; $\mu$ Bondasphere C8 column from Nihon Waters Ltd., Tokyo; YMC A-302 S-5 ODS column from Yamamura Kagaku, Kyoto; reagents for gas-phase sequencer from Applied Biosystems, CA. All other chemicals were of analytical grade or of the highest quality commercially available. Factor $I X /$ factor $X$-binding protein ( $I X / X-b p)$ purified from the venom of Trimeresurus flavoviridis (18) was a gift of Dr. Hideko Atoda, Meiji College of Pharmacy.

$$
\text { Purification of } R V V-X-R V V-X \text { was isolated from the }
$$

venom of Vipera russelli siamensis by minor modifications of published methods (11), consisting of two steps, gel filtration on Sephadex G-75 and ion-exchange chromatography on DEAE-Sepharose CL-6B . 

SDS-PAGE was carried out by the method of Laemmli (19). The reference protein kit for molecular weight estimate was obtained from Pharmacia Fine Chemicals, Uppsala. Preparation of Heavy and Light Chains - The purified RVV-X was reduced and S-pyridylethylated as previously described (17). The resulting product was subjected to gel filtration HPLC on a G3000SW column equilibrated with $0.1 \mathrm{M}$ sodium phosphate buffer, $\mathrm{pH} 6.0$, containing $6 \mathrm{M}$ guanidine hydrochloride and $1 \mathrm{mM}$ EDTA. The light chain fraction was subjected to reversed phase HPLC on a Vydac 214 TP5415 column in $0.1 \%$ trifluoroacetic acid with acetonitrile gradient elution.

CNBr and Proteolytic Digestion The sample was cleaved with $\mathrm{CNBr}$ as previously described (17). The sample in $50 \mathrm{mM}$ TrisHCl buffer, pH 8.0, containing $4 \mathrm{M}$ urea was digested with lysyl endopeptidase $(\mathrm{E} / \mathrm{S}=1 / 50, \mathrm{~mol} / \mathrm{mol})$ at $37^{\circ} \mathrm{C}$ for $12 \mathrm{~h}$. The samples in $50 \mathrm{mM}$ Tris-HCl buffer, $\mathrm{pH} 8.0$, containing $2 \mathrm{M}$ urea were digested with endoproteinase Asp-N (E/S=1/50, $\mathrm{mol} / \mathrm{mol})$ for $13 \mathrm{~h}$, trypsin $(E / S=1 / 20, \mathrm{~mol} / \mathrm{mol})$ for $12 \mathrm{~h}$, or chymotrypsin $(E / S=1 / 50$, $\mathrm{mol} / \mathrm{mol}$ ) for $6 \mathrm{~h}$ at $37{ }^{\circ} \mathrm{C}$. The samples in $0.1 \mathrm{M} \mathrm{NH}_{4} \mathrm{HCO}_{3}$ buffer, $\mathrm{pH}$ 8.0 , containing $2 \mathrm{M}$ urea were digest'ed with Staphylococcus aureus V8 protease $(\mathrm{E} / \mathrm{S}=1 / 70, \mathrm{~mol} / \mathrm{mol})$ at $37{ }^{\circ} \mathrm{C}$ for $17 \mathrm{~h}$. For limited proteolysis, non-reduced RVV-X in 50 mM Tris-HCl buffer, $\mathrm{pH} 7.5$, containing $0.1 \mathrm{M} \mathrm{NaCl}$ and $10 \mathrm{mM}$ EDTA was digested with trypsin (E/S=1/50, $\mathrm{mol} / \mathrm{mol})$ at $37{ }^{\circ} \mathrm{C}$ for $3 \mathrm{~h}$.

Peptide Purification The peptides generated after $\mathrm{CNBr}$ cleavage of Pe-heavy chain were separated by reversed phase HPLC after gel filtration HPLC on a column of G2000SW, equilibrated with $0.1 \mathrm{M}$ phosphate buffer, $\mathrm{pH} 6.0$, containing $6 \mathrm{M}$ guanidine 
hydrochloride and $1 \mathrm{mM}$ EDTA. All other peptides were purified by reversed phase HPLC as previously described (17).

Amino Acid Analysis and Sequence Determination The amino acid analyses of the Pe-proteins were performed by ionexchange chromatography in a Hitachi model L-8500 high speed amino acid analyzer after hydrolysis with $5.7 \mathrm{M} \mathrm{HCl}$ containing 0.2 \& phenol at $110{ }^{\circ} \mathrm{C}$ for 24,48 , and $72 \mathrm{~h}$ by the method of spackman et al. (20). Tryptophan was determined by hydrolysis in $3 \mathrm{M}$ mercaptoethanesulfonic acid (21). The CNBr fragments derived from Pe-heavy chain were analyzed using a Hitachi L-8500 amino acid analyzer after hydrolysis with $5.7 \mathrm{M} \mathrm{HCl}$ containing $0.2 \%$ phenol at $110{ }^{\circ} \mathrm{C}$ for $24 \mathrm{~h}$. Other peptides were analyzed using reversedphase HPLC of phenylthiocarbamoyl derivatives (22), using a Waters PICO-TAG system, after hydrolysis with $5.7 \mathrm{M} \mathrm{HCl}$ containing 1 \% phenol at $110{ }^{\circ} \mathrm{C}$ for $20 \mathrm{~h}$. Automated sequence analyses were performed with an Applied Biosystems 477A protein sequencer, as described by Hewick et al. (23), with an Applied Biosystems model 120A PTH analyzer.

Determination of the Carboxyl-Terminal Amino Acid The carboxyl-terminal amino acid was determined by the vapor-phase hydrazinolysis method as previously described $(16,24)$.

Platelet Aggregation Assay Platelet aggregation assay was performed in human platelet-rich plasma (PRP) according to the methods of Moroi (25). Fifty ml of whole human blood ( 9 parts) from a healthy donor was collected in $3.8 \%$ (wt/vol) sodium citrate (1 part). Blood was centrifuged (1,800 rpm for $5 \mathrm{~min}$ ) at room temperature and PRP was collected. Platelet-poor plasma (PPP) was prepared from the remaining blood by centrifugation $(3,000 \mathrm{rpm}$ for $10 \mathrm{~min})$ at room temperature. PRP plus solution of 
RVV-X in Tris-HCl buffer saline, $\mathrm{pH} 7.5$ or buffer alone was incubated for $3 \mathrm{~min}$ in a Chrono-log Aggregometer at $37^{\circ} \mathrm{C}$. An aggregating agent (collagen, $1 \mu \mathrm{g} / \mathrm{ml}$ or $\mathrm{ADP}, 10 \mu \mathrm{M}$ ) was added and the light transmittance was recorded.

Effect of IX/X-bp on the RVV-X-mediated Activation of Factor $x$ - Factor $\mathrm{X}(3.6 \mu \mathrm{M})$ was incubated with the various concentrations of IX/X-bp $(0-7.2 \mu \mathrm{M})$ in $50 \mathrm{mM}$ Tris-HCl, $\mathrm{pH} 8.0$, containing $10 \mathrm{mM} \mathrm{CaCl} 2$ for $1 \mathrm{~min}$ at $25{ }^{\circ} \mathrm{C}$. Then, $\mathrm{RVV}-\mathrm{X}$ ( $3.6 \mathrm{nM}$ ) was added. After $1 \mathrm{~min}, 10 \mu \mathrm{l}$ aliquots of the reaction mixture were added to the substrate solution, which consisted of $600 \mu 1$ of $50 \mathrm{mM}$ Tris-HCl containing $0.1 \mathrm{mg} / \mathrm{ml}$ bovine serum albumin, $2 \mathrm{mM}$ EDTA and $0.1 \mathrm{M} \mathrm{NaCl}$, and $10 \mu \mathrm{l}$, of $5 \mathrm{mM} N^{\alpha}$-benzyloxycarbonyl-Lpyroglutamyl-Gly-Arg-4-methylcoumaryl-7-amide in $\mathrm{N}, \mathrm{N-}$ dimethylformamide. The released 7-amino-4-methylcoumarin was measured fluorometrically with excitation at $380 \mathrm{~nm}$ and emission at $440 \mathrm{~nm}$.

Nomenclature of the Peptides Peptides are designated by a serial number prefixed by a letter. The letters indicated the type of digestion: $M, \operatorname{CNBr} ; \mathrm{K}$, lysyl endopeptidase; D, endoproteinase Asp-N; T, trypsin; E, V8 protease; C, chymotrypsin. The numbers in the peptide designation do not correspond to the order of their elution in HPLC, but rather to their positions in the protein sequence, starting from the aminoterminus. 


\section{RESULTS}

Preparation of Heavy and Light'chains and Their Amino Acid Compositions Highly purified RVV-X was reduced and $S-$ pyridylethylated $(\mathrm{Pe})$, and subsequently subjected to gel filtration (Fig. 1). As shown in the inset of Fig. 1, the SDS-PAGE analyses of two peaks revealed that the former peak (peak HC) contains a homogeneous heavy chain (Mr 59,000) and the latter peak (peak LC) contains heterogeneous light chains (Mr 18,000 and 21,000) with a doublet. These two heterogeneous light chains were further separated by reversed phase HPLC (Fig. 2). Highly purified two light chains (the inset of Fig, 2) were designated as LC1 and LC2. In the present study, the major component, LC1, was selected as being representative and was used for sequence analyses. The amino acid compositions of heavy and light chains are shown in Table 1. Although LC1 and LC2 have similar amino acid compositions, their peptide maps after digestions with lysyl endopeptidase differed (data not shown), indicating a polymorphism with several residues. The heavy and light chains contained glucosamine, but not galactosamine (Table 1).

$$
\text { Sequence Analyses of Heavy Chain The automated Edman }
$$

degradation of Pe-heavy chain established the amino-terminal 31 residues starting with the sequence of Leu-Val-Ser-Thr-Ser-Ala- as shown in Fig. 3. In addition to this sequence, we could identify the minor amino-terminal sequences Val-Ser-Thr-Ala- and Ser-Thr-Alastarting at positions 2 and 3, respectively. The Pe-heavy chain was cleaved with $\mathrm{CNBr}$ and the resulting peptides were separated by gel filtration (Fig. 4). M5 and M12, and M3, M4, and M6 were further separated by reversed phase HPLC on a column of Cosmosil 5C4 300 (data not shown). The amino acid compositions and amino-terminal 
sequences of the isolated peptides are shown in Table II and Fig. 3. M2-3 and M7-8 were yielded by incomplete cleavage at a Met-Thr bond (residues 90-91) and a Met-Ser bond (residues 169-170), respectively. To overlap the CNBr fragments, the Pe-heavy chain was digested, separately, with lysyl endopeptidase and endoproteinase Asp-N. The digest with lysyl endopeptidase was separated by reversed phase HPLC on a column of Cosmosil 5C4 300 (data not shown). The amino acid compositions and amino-terminal sequences of the isolated peptides are shown in Table III and Fig. 3. The digest with endoproteinase Asp- $\mathrm{N}$ was separated by reversed phase HPLC on a column of $\mu$ Bondasphere C8 and several fractions containing more than two peptides were further subjected to rechromatography on a column of Chemcosorb 5-ODS-H. The amino acid compositions of these peptides were determined (data not shown) and the amino-terminal sequences of 12 peptides were analyzed (Fig. 3). To complete the sequence of Peheavy chain, large fragments, M7-8, M9 and K4 were subdigested with chymotrypsin, lysyl endopeptidase, and trypsin, respectively, and the resulting peptides were isolated by reversed phase HPLC. The amino acid compositions of these peptides were determined (data not shown) and the amino-terminal sequences of overlapping peptides were analyzed (Fig. 3). The heavy chain consisted of 427 amino acid residues with 4 asparagine-linked sugar chains at positions 28, 69, 163 and 183 (Fig. 3).

Sequence Analyses of Light Chain (LC1) The aminoterminal sequence analysis of Pe-LC1 established the first 38 residues with the exception of several unidentified residues. Pe-LC1 was primarily digested with lysyl endopeptidase and the resulting peptides were separated by reversed phase HPLC (Fig. 5). The amino acid compositions and amino-terminal sequences of the isolated 
peptides are shown in Table IV and Fig. 6. Large fragment K5 was further subdigested with V8 protease, and the peptide K5E3 isolated by reversed phase HPLC was used to complete the sequence of $\mathrm{K} 5$. To overlap these peptides, Pe-LC1 was cleaved with CNBr. The resulting peptides were separated by reversed phase HPLC on a column of YMC A302 ODS after gel filtration on a column of G2000SW (data not shown). The amino acid compositions and amino-terminal sequences of the isolated peptides are shown in Table V and Fig. 6. M3-4 was yielded by incomplete cleavage at a Met-Ile bond (residues 101-102) in the same manner as previously observed (16). The carboxyl-terminal amino acid of LC1 was determined to be phenylalanine residue by vapor-phase hydrazinolysis method $(0.17 \mathrm{~mol} / \mathrm{mol}$ of protein, uncorrected). The light chain LC1 consisted of 123 amino acid residues containing an asparagine-linked sugar chain at position 24 (Fig. 6).

Carbohydrate-linked Asparagine Residues — The PTH derivatives at position 24 in the light chain LC1 and at positions $28,69,163$ and 183 in the heavy chain, were not identified by sequence analysis. These are very likely carbohydrate-linked Asn residues, since they have a potential $-\mathrm{X}-\mathrm{Th} / \mathrm{Ser}$ sequence, which is known as a consensus signal sequence for the attachment of carbohydrate to asparagine. In fact, the composition analyses of the fragments containing these residues indicated the presence of glucosamines in each fragment. The reasonably high yields for Thr and Ser residues in the entire sequence and the composition analyses of all the fragments, including intact protein, indicated no o-linked carbohydrate chains in RVV-X.

$$
\text { Titration of the Free SH-Group The concentration of a }
$$

free SH-group was determined in the presence of $6 \mathrm{M}$ guanidine hydrochloride and $10 \mathrm{mM}$ EDTA by the method of Ellman (26) and also 
incorporation of 4-vinylpyridine in the absence and presence of the denaturant containing $6 \mathrm{M}$ guanidine hydrochloride and $10 \mathrm{mM}$ EDTA. No free SH-group could be detected in the non-reduced RVV-X by the two methods with Ellman's reagent (5,5'-dithiobis (2-nitrobenzoic acid)) and with S-pyridylethylation.

Platelet Aggregation Inhibitory Activity of RVV-X Since the middle portion of the heavy chain (residues 212-301) showed a remarkable sequence similarity to those of venom platelet aggregation inhibitors, disintegrins (see Fig. 7), we tested the platelet aggregation inhibitory activity of RVV-X. As shown in Fig. 8, RVV-X dose-dependently inhibited collagen- and ADP- induced platelet aggregations. The percentages of inhibition on collagen- and ADPinduced platelet aggregations were $44 \%$ and $85 \%$, respectively, at a concentration of $310 \mathrm{nM}$.

Effect of $I X / X-b p$ on the RVV-X-mediated Activation of Factor $X$ We also examined the effect of IX/X-bp on the RVV-X-mediated activation of factor $\mathrm{x}$, since the amino acid sequence of the light chain LCl is similar to that of IX/X-bp (Fig. 9). As shown in Fig. 10, IX/X-bp inhibited the activation of factor $\mathrm{x}$, in a dose-dependent manner. The $86 \%$ inhibition was observed in the presence of an equal molar ratio of $\mathrm{IX} / \mathrm{X}-\mathrm{bp}$ to factor $\mathrm{X}$. 


\section{DISCUSSION}

We reported the primary structures of several hemorrhagic and non-hemorrhagic metalloproteinases isolated from the venoms of Trimeresurus flavoviridis (14-16) and Crotalus ruber ruber (17), the objective being to elucidate the molecular mechanism of hemorrhage, the major symptoms following the bite of crotalid and viperid snakes. These metalloproteinases belong to a new metalloproteinase subfamily, all of which contain a zinc-chelation sequence His-Glu-X-X-His but have no significant sequence homology with any known metalloproteinases beyond this region (17). The most exciting finding in this series of studies is that high molecular mass hemorrhagic metalloproteinase HR1B ( $\mathrm{Mr} 60,000)$ contains the cysteinerich carboxyl-terminal half (residues 204-416) which includes the disintegrin-like structure, in addition to the amino-terminal half metalloproteinase domain (residues 1-203) similar to low molecular mass metalloproteinases in sequences (16). Disintegrins isolated from snake venoms are potent platelet aggregation inhibitors and are cysteine-rich and Arg-Gly-Asp (RGD)-containing polypeptides (total 48-84 residues) (see Refs. 27 and 28 for review).

As shown in Fig. 7, the entire amino acid sequence of the RVV$X$-heavy chain resembles that of HR1B (53 \% sequence identity). The sequence of its amino-terminal half (residues 1-205) is also similar to those of the low molecular mass metalloproteinases HR2a, HT-2, Htd (29) and $\mathrm{H}_{2}$-proteinase (40-43 \& sequence identity), forming a metalloproteinase domain of RVV-X (Fig. 7A). Like others, this domain of RVV-X contains the His-Glu-X-X-His-sequence (residues 145149), in which His 145 and His 149 , and Glul46 are believed to be two of the zinc-chelating ligands and one of the catalytic residues, as 
of the zinc-chelating ligands and one of the catalytic residues, as discussed elsewhere (17). It is of interest that HR1B, HR2a, HT-2 and Ht-d cause a localized hemorrhage, but $\mathrm{H}_{2}$-proteinase does not, even though all of them have a similar structure. In this regard, we first focused on structural elements associated with the hemorrhagic activity. Since the purified RVV-X did not express hemorrhagic activity (data not shown), we compared the amino acid sequences of two non-hemorrhagic metalloproteinases RVV-X and $\mathrm{H}_{2}-$ proteinase with those of hemorrhagic metalloproteinases HR1B, HR2a, HT-2, and Ht-d. There are five amino acid residues shared by hemorrhagic metalloproteinases but not by non-hemorrhagic RVV-X and $\mathrm{H}_{2}$-proteinase (boxed residues in Fig. $7 A$ ). These residues might be closely associated with the hemorrhagic activity.

The middle region of the RVV-X-heavy chain (residues 212-301) shows a high percentage of sequence identity to the RGD-sequence containing disintegrins, scored $59 \%$ for trigramin (30), 38 \& for echistatin (31), $60 \%$ for bitistatin (32) and $55 \%$ for barbourin (33). Although RVV-X as well as the disintegrin family is able to inhibit platelet aggregation (Fig. 8), the RGD-sequence is absent and is substituted for by Arg-Asp-Glu in the corresponding region (Fig. 7). Similar results have been found in HR1B with a Glu-SerGlu substitution (16). The platelet aggregation inhibitory activity of RVV-X apparently resides within the disintegrin-like domain, based on the same notion as that discussed in the case of HR1B (see Ref. 16). However, there is a possibility that platelet aggregation inhibitory activity could be due to some of the proteolytic activity or the lectin like activity, since we have used the whole RVV-X for that experiment (Fig. 4). All of twenty-five disintegrins so far isolated contain the RGD-sequence, which is believed to be an 
interaction site for several integrins, with one exception, barbourin containing Lys-GIy-Asp instead (33). The substitution in barbourin is suggested to impart integrin specificity to barbourin, since it has been isolated as a specific antagonist for glycoprotein IIb/IIIa, while other RGD-containing disintegrins interact with various integrins of $\beta_{1}$ and $\beta_{3}$ families, such as the vitronectin receptor(s) and fibronectin receptor. The integrin specificities of RVV-X and HR1B remain to be determined. Equally important in future experiments is to account for the in vivo relevance of this platelet aggregation inhibitory effect of RVV-X.

The carboxyl-terminal region of RVV-X-heavy chain (residues 302-427) following the disintegrin domain contains numerous cysteine residues. Computerized homology searches revealed that the cysteine-rich region of $\mathrm{HR} 1 \mathrm{~B}$ is the sole structure homologous to this region, the function of this region is unclear.

Interestingly, the light chain (LC1) of RVV-X shows a striking similarity with $\mathrm{C}$-type lectin-like structure $\left(\mathrm{Ca}^{2+}\right.$-dependent carbohydrate recognition domain). Numerous proteins isolated from snakes and mammals were seen to contain this structure (34), as shown in Fig. 9. The sequence similarity between RVV-X-light chain (LC1) and rattlesnake lectin (35), phospholipase A2 inhibitor A subunits (36) and factor IX/factor X-binding protein A chain (37), mouse lymphocyte homing receptor (38), rat Kupffer cell receptor (39), rat mannose-binding protein A (40), human lymphocyte IgE receptor (41), human tetranectin (42) and human pulmonary surfactant associated protein $(43)$ is $27,13,33,17,22,10,22,15$ and 108, respectively. Factor $\mathrm{IX} /$ factor $\mathrm{X}$-binding protein $(\mathrm{IX} / \mathrm{X}-\mathrm{bp})$ is the most interesting among these proteins having the C-type lectin structure, since $\mathrm{IX} / \mathrm{X}-\mathrm{bp}$ and $\mathrm{RVV}-\mathrm{X}$ share several key 
characteristics, in addition to sequence similarity. IX/X-bp has been purified as an anticoagulant protein and it binds specifically to factors $I X$ and $x$, but not to other vitamin $K$-dependent coagulation factors (18). This functional activity of $I X / X-b p$ as well as RVV-X is dependent on $\mathrm{Ca}^{2+}$ and the amino-terminal Gladomains of factors IX and $X(13,18)$. IX/X-bp is able to inhibit the $\mathrm{RVV}-\mathrm{X}$-catalyzed factor $\mathrm{X}$ activation, in a dose dependent manner (Fig. 10), thereby indicating that IX/X-bp occupies a RVV-Xrecognized site on factor $x$. Taken together, it is suggested that the light chain of RVV-X might play an important role in the binding of $\mathrm{RVV}-\mathrm{X}$ with factor $\mathrm{X}$, and that it recognizes the Gla-domain of factor $\mathrm{X}$. There are two potential roles of the exogenous $\mathrm{Ca}^{2+}$, which is required for the factor $\mathrm{x}$ activating activity but not for the proteolytic activity of RVV-X: First, the C-type lectin-like light chain of RVV-X requires $\mathrm{Ca}^{2+}$ for its function, and second, $\mathrm{Ca}^{2+}$ induces conformational change of the Gla-domain (44), an event which might be a prerequisite for recognition by RVV-X and/or for concomitant conformational change of factor $\mathrm{X}$ susceptible to RVV-X. Since no free SH-group was detected in RVV-X, all the cysteine residues in RVV-X must be linked with disulfide bridges. Six of seven half-cystines in the RVV-X-light chain (LC1) are conserved in several C-type lectin-like structures in which the disulfide bridge locations have been determined, such as rattlesnake lectin (35), acorn banacle lectin (45), sea urchin echinoidin (46) and human tetranectin (42). Based on these data, three intrachain disulfide bridges of RVV-X-LC1 could be assigned by homology as follows: Cys4Cys 15, Cys $32-$ Cys 121 , and Cys98-Cys113. Therefore, the remaining Cys 77 might be involved in an interchain disulfide bridge between the heavy and the light chains. Since 34 out of 37 half-cystines in 
the RVV-X-heavy chain are located at the same positions as those of HR1B (16), three other cysteine residues Cys 27, Cys 63 and Cys 389 are unique in the RVV-X-heavy chain (Fig. 7). Among them, Cys 389 is suggested to be the half-cystine participating to form an interchain disulfide bridge between the heavy and the light chains, since trypsin cleaves the non-reduced heavy chain at several peptide bonds only in the amino-terminal half of the heavy chain, under the conditions described in Materials and Methods, and since the remaining carboxyl-terminal half starting at residue 209 of the heavy chain is still bridged with the light chain (data not shown). In this regard, the amino-terminal metalloproteinase domain composing RVV-X is not linked through disulfide bridges to any other domains located in the carboxyl-terminal portion. Six of eight half-cystines in this domain are conserved in HR2a and $\mathrm{H}_{2}-$ proteinase, in which the disulfide bridge locations have been determined $(14,15)$. Therefore, three intra-chain disulfide bridges of this domain could be assigned by homology as follows: Cys 120 Cys200, Cys160-Cys184, and Cys162-Cys167, and the remaining Cys 27 and Cys 63 might also form an intrachain disulfide bridge in the metalloproteinase domain. Regarding the disulfide bonding patterns of the disintegrin-like domain in RVV-X, it has not been established. However, all the positions of cysteine residues found in the disintegrin-like domain of RVV-X except for Cys278 are the same as those of kistrin (47), in which the disulfide locations have recently been deduced from 2D NMR studies (48).

In Fig. 11, the gross structure of RVV-X is illustrated with other known members of snake venom metalloproteinase family. The putative precursor protein of trigramin is also a member of this family ${ }^{1}$. These findings show the structural and evolutional 
relationship among these proteins, although each protein has diversed functional activity. These mosaic structures are also analogous to those of mammalian blood coagulation serine proteinases (51) and matrix metalloproteinases (50). While a common domain with apparently distinct functions can be defined among these proteinases, additional domains unique to individual proteinases will have specific functions. It is presumed that RVV-X evolutionally acquired substrate specificity as a result of assembly of metalloproteinase domain and c-type lectin-like domain.

\section{FOOTNOTE}

${ }^{1}$ Neeper and Jacobson recently reported the sequence of CDNA for trigramin, but they did not translate a large nucleotide sequence, which is present upstream from the coding region for 72 residues mature trigramin (49). We translated the nucleotide sequence of this region into the corresponding amino acid residues and found that such a region represents a metalloproteinase structure similar to the venom metalloproteinase family (Fig. 12). The trigramin precursor contains an additional 172 residues between the signal sequence and metalloproteinase domain. This portion might be a propeptide found in inactive pro-forms of mammalian matrix metalloproteinases (50). 


\section{REFERENCES}

1. Iwanaga, S., and Suzuki, T. (1979) in Handbook of Experimental Pharmacology (Lee, C.-Y., ed), Vol. 52, pp. 61-158, SpringerVerlag, New York

2. Morita, T., and Iwanaga, S. (1981) Methods Enzymol. 80, 303-311

3. Kisiel, W., and Canfield, W.M. (1981) Methods Enzymol. 80, $275-285$

4. Pirkle, H., and Stocker, K. (1991) Thromb. Haemostas. 65, 444450

5. Rosing, J., and Tans, G. (1991) Thromb. Haemostas. 65, 627-630

6. Tokunaga, F., Nagasawa, K., Tamura, S., Miyata, T., Iwanaga, S., and Kisiel, W. (1988) J. Biol. Chem. 263, 17471-17481

7. Furie, B.C., and Furie, B. (1976) Methods Enzymol. 45, 191-205

8. Fujikawa, K., Legaz, M.E., and Davie, E.W. (1972) Biochemistry $11,4892-4899$

9. Di Scipio, R.G., Hermodoson, M.A., and Davie, E.W. (1977) Biochemistry $16,5253-5260$

10. Jackson, C.M., Gordon, J.G., and Hanahan, D.J. (1971) Biochem. Biophys. Acta. 252, 255-261

11. Kisiel, W., Hermodoson, M.A., and Davie, E.W. (1976) Biochemistry 15, 4901-4906

12. Amphlett, G.W., Byrne, R., and Castellino, F.J. (1982) Biochemistry 21, 125-132

13. Skogen, W.F., Bushong, D.S., Johnson, A.E., and Cox, A.C. (1983) Biochem. Biophys. Res. Communs. 111, 14-20

14. Miyata, T., Takeya, H., Ozeki, Y., Arakawa, M., Tokunaga, F., Iwanaga, S., and Omori-Satoh, T. (1989) J. Biochem. (Tokyo) $105,847-853$ 
15. Takeya, H., Arakawa, M., Miyata, T., Iwanaga, S., and OmoriSatoh, T. (1989) J. Biochem. (Tokyo) 106, 151-157

16. Takeya, H., Oda, K., Miyata, T., Omori-Satoh, T., and Iwanaga, S. (1990) J. Biol. Chem. 265, 16068-16073

17. Takeya, H., Onikura, A., Nikai, T., Sugihara, H., and Iwanaga, S. (1990) J. Biochem. (Tokyo) 108, 711-719

18. Atoda, H., and Morita, T. (1989) J. Biochem. (Tokyo) 106, 808813

19. Laemmli, U.K. (1970) Nature 227, 680-685

20. Spackman, D.H., Stein, W.H., and Moore, S. (1958) Anal. Chem. $30,1190-1206$

21. Penke, B., Ferenczi, R., and Kovacs, K. (1974) Anal. Biochem. $60,45-50$

22. Bidlingmeyer, B.A., Cohen, S.A., and Tarvin, T.L. (1984) J. Chromatogr. 336, 93-104

23. Hewick, R.M., Hunkapiller, M.W., Hood, L.E., and Dreyer, W.J. (1981) J. Biol. Chem. 256, 7990-7997

24. Yamamoto, A., Toda, H., and Sakiyama, F. (1989) J. Biochem. (Tokyo) $106,552-554$

25. Moroi, M., Jung, S.M., Okuma, M., and Shinmyozu, K. (1989) J. Clin. Invest. 84, 1440-1445

26. Ellman, G.L. (1959) Arch. Biochem. Biophys. 82, 70-77

27. Gould, R.J.. Polokoff, M.A., Friedman, P.A., Huang, T.-F., Holt, J.C., Cook, J.J., and Niewiarowski, S. (1990) Proc. Soc. Exp.

Biol. Med. 195, 168-171

28. Teng, C.-M., and Huang, T.-F. (1991) Thromb. Haemostas. 65, $624-626$

29. Shannon, J.D., Baramova, E.N., Bjarnason, J.B., and Fox, J.W. (1989) J. Biol. Chem. 264, 11575-11583 
30. Hung, T.-F., Holt, J.C., Kirby, E.P., and Niewiarowski, S. (1989) Biochemistry 28, 661-666

31. Gan, Z.-R., Gould, R.J., Jacobs, J.W., Friedman, P.A., and Polokoff, M.A. (1988) J. Biol. Chem. 263, 19827-19832

32. Shebuski, R.J., Ramjit, D.R., Bencen, G.H., and Polokoff, M.A. (1989) J. Biol. Chem. 264, 21550-21556

33. Scarborough, R.M., Rose, J.W., Hsu, M.A., Phillips, D.R., Fried V.A., Campbell, A.M., Hannizzi, L., and Charo, I.F. (1991) J. Biol. Chem. 266, 9359-9362

34. Drickamer, K. (1988) J. Biol. Chem. 263, 9557-9560

35. Hirabayashi, J., Kusunoki, T., and Kasai, K. (1991) J. Biol. Chem. 266, 2320-2326

36. Inoue, S., Kogaki, H., Ikeda, K., Samejima, Y., and Omori-Satoh, T. (1991) J. Biol. Chem. 266, 1001-1007

37. Atoda, H., Hyuga, M., and Morita, T. (1991) J. Biol. Chem. 266, $14903-14911$

38. Lasky, L.A., Singer, M.S., Yednock, T.A., Dowbenko, D., Fennie, C., Rodriguez, H., Nguyen, T., Stachel, S., and Rosen, S.D. (1989) Cell 56, 1045-1055

39. Hoyle, G.W., and Hill, R.L. (1988) J. Biol. Chem. 263, 748774.92

40. Drickamer, K., Dordal, M.S., and Reynolds, L. (1986) J. Biol. Chem. 261, 6878-6887

41. Kikutani, H., Inui, S., Sato, R., Barsumian, E.L., Owaki, H., Yamasaki, K., Kaisho, T., Uchibayashi, N., Hardy, R.R., Hirano, T., Tsunasawa, S., Sakiyama, F.', Suemura, M., and Kishimoto, T. (1986) Cel1 47, 657-665

42. Fuhlendorff, J., Clemmensen, I., and Magnusson, S. (1987) Biochemistry 26, 6757-6764 
43. Floros, J., Steinbrink, R., Jacobs, K., Phelps, D., Kriz, R., Recny, M., Sultzman, L., Jones, S., Taeusch, H.W., Frank, H.A., and Fritsch, E.F. (1986) J. Biol. Chem. 261, 9029-9033

44. Higashi, S., Kawabata, S., Nishimura, H., Funasaki, H., Ohyama, S., Miyamoto, S., Funatsu, A., and Iwanaga, S. (1990) J.

Biochem. (Tokyo) 108, 654-662

45. Muramoto, K., and Kamiya, H. (1986) Biochem. Biophys. Acta. $874,285-295$

46. Giga, Y., Ikai, A., and Takahashi, K. (1987) J. Biol. Chem. 262, $6197-6203$

47. Dennis, M.S., Henzel, W.J., Pitti, R.M., Lipari, M.T., Napier, M.A., Deisher, T.A., Bunting, S., and Lazarus, R.A. (1990) Proc. Natl. Acad. Sci. USA 87, 2471-2475

48. Adler, M., Lazarus, R.A., Dennis, M.S., and Wagner, G. (1991) Science 253, 445-448

49. Neeper, M.P., and Jacobson, M.A. (1990) Nucleic Acids Research 18,4255

50. Van Wart, H.E., and Birkedal-Hansen, H. (1990) Proc. Natl. Acad. SCi. USA 87, 5578-5582

51. Davie, E.W., Fujikawa, K., and Kisiel, W. (1991) Biochemistry $30,10363-10370$ 
Table I

Amino acid compositions of RVV-X-heavy (HC), and -light chains (LC1 and LC2a)

\begin{tabular}{|c|c|c|c|c|c|}
\hline $\begin{array}{l}\text { Amino } \\
\text { acid }\end{array}$ & $\mathrm{HC}$ & Sequence & LC 1 & Sequence & LC2 \\
\hline & & \multicolumn{2}{|c|}{ Residues/molecule } & $\underline{-}$ & \\
\hline Asp & 62.8 & 63 & 12.0 & 12 & 11.4 \\
\hline Thrb & 20.5 & 21 & 5.2 & 5 & 3.9 \\
\hline serb & 30.7 & 29 & 9.4 & 9 & 11.5 \\
\hline Glu & 40.1 & 40 & 15.8 & 16 & 16.5 \\
\hline Pro & 20.1 & 20 & 3.6 & 3 & 4.3 \\
\hline Gly & 26.9 & 27 & 6.6 & 6 & 5.4 \\
\hline Ala & 23.1 & 22 & 5.4 & 5 & 4.7 \\
\hline Valc & 21.4 & 22 & 7.6 & 7 & 7.5 \\
\hline Met & 10.7 & 11 & 5.2 & 4 & 4.4 \\
\hline$I 1 e^{C}$ & 20.9 & 22 & 5.2 & 5 & 4.2 \\
\hline Leu & 26.5 & 25 & 10.0 & 10 & 8.6 \\
\hline Tyr & 14.1 & 14 & 5.0 & 5 & 5.2 \\
\hline Phe & 14.9 & 14 & 5.0 & 5 & 8.0 \\
\hline Lys & 25.6 & 26 & 11.1 & 11 & 9.3 \\
\hline $\mathrm{His}$ & 10.7 & 10 & 3.7 & 4 & 3.5 \\
\hline Trpd & 3.4 & 3 & 3.5 & 6 & 2.6 \\
\hline $\mathrm{Pec}$ & 34.2 & 37 & 5.5 & 7 & 5.2 \\
\hline Arg & 20.1 & 21 & 3.3 & 3 & 6.7 \\
\hline $\mathrm{GlCNH}_{2} \mathrm{f}$ & + & & + & & + \\
\hline Total & 426.7 & 427 & 123.1 & 123 & 122.9 \\
\hline
\end{tabular}

acalculated from average values obtained from 24,48 and 72 h hydrolysates.

bextrapolation to $0 \mathrm{~h}$.

cTaken from 72 h hydrolysis.

$\mathrm{d}_{\text {Taken }}$ from $24 \mathrm{~h}$ hydrolysis with $3 \mathrm{M}$ mercaptoethanesulfonic acid. es-pyridylethylcysteine.

$\mathrm{f} \overline{\mathrm{G}} \mathrm{lucosamine}$. 
Table II

Amino acid compositions of cyanogen bromide peptides derived from RVV-X-heavy chaina

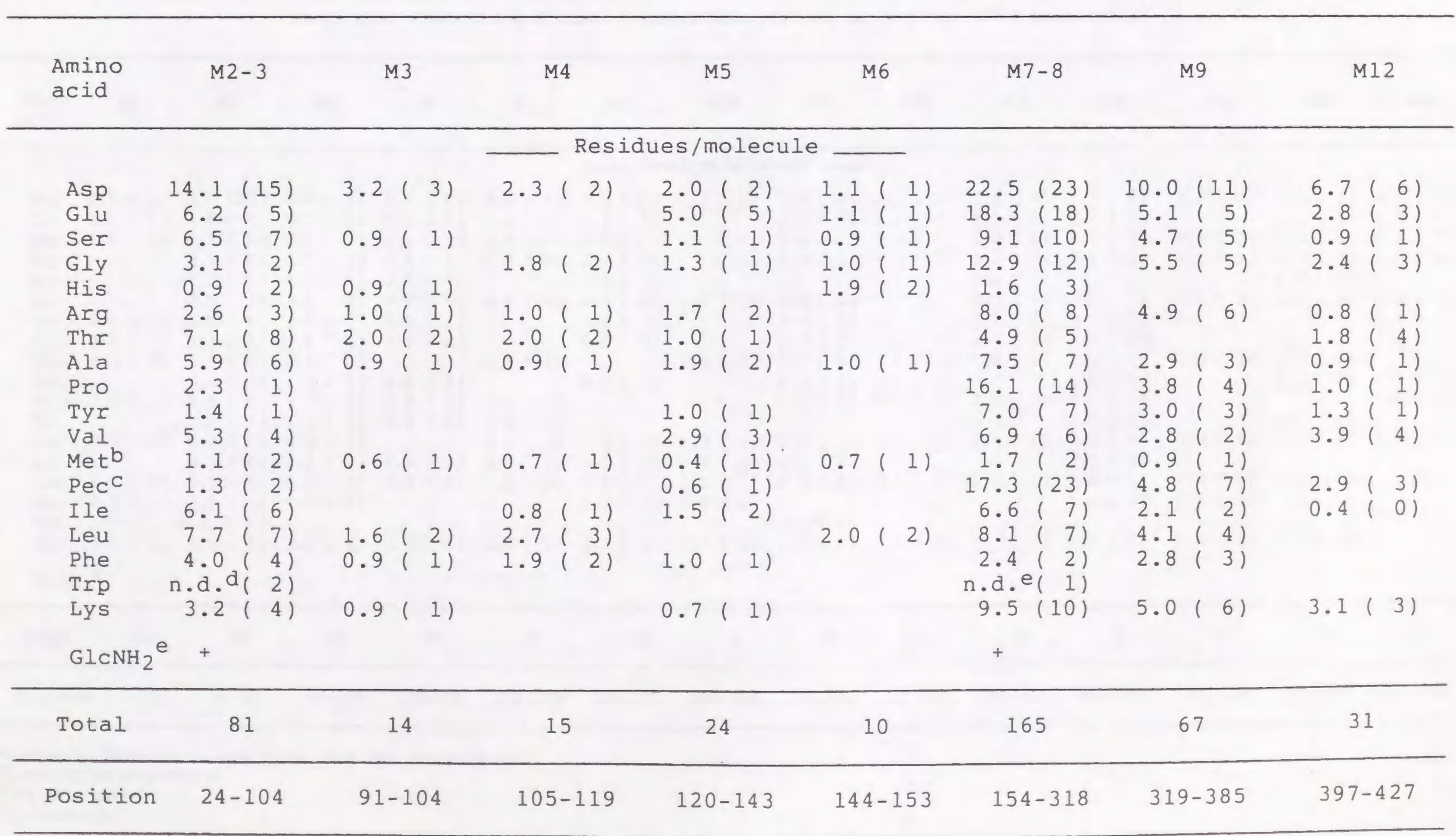

avalues in parentheses are taken from the sequence data.

betermined as homoserine.

CS-Pyridylethylcysteine.

$\mathrm{d} \overline{\text { Not }}$ determined.

eglucosamine. 
Table III

Amino acid compositions of lysyl endopeptidase peptides derived from RVV-X-heavy chain ${ }^{a}$

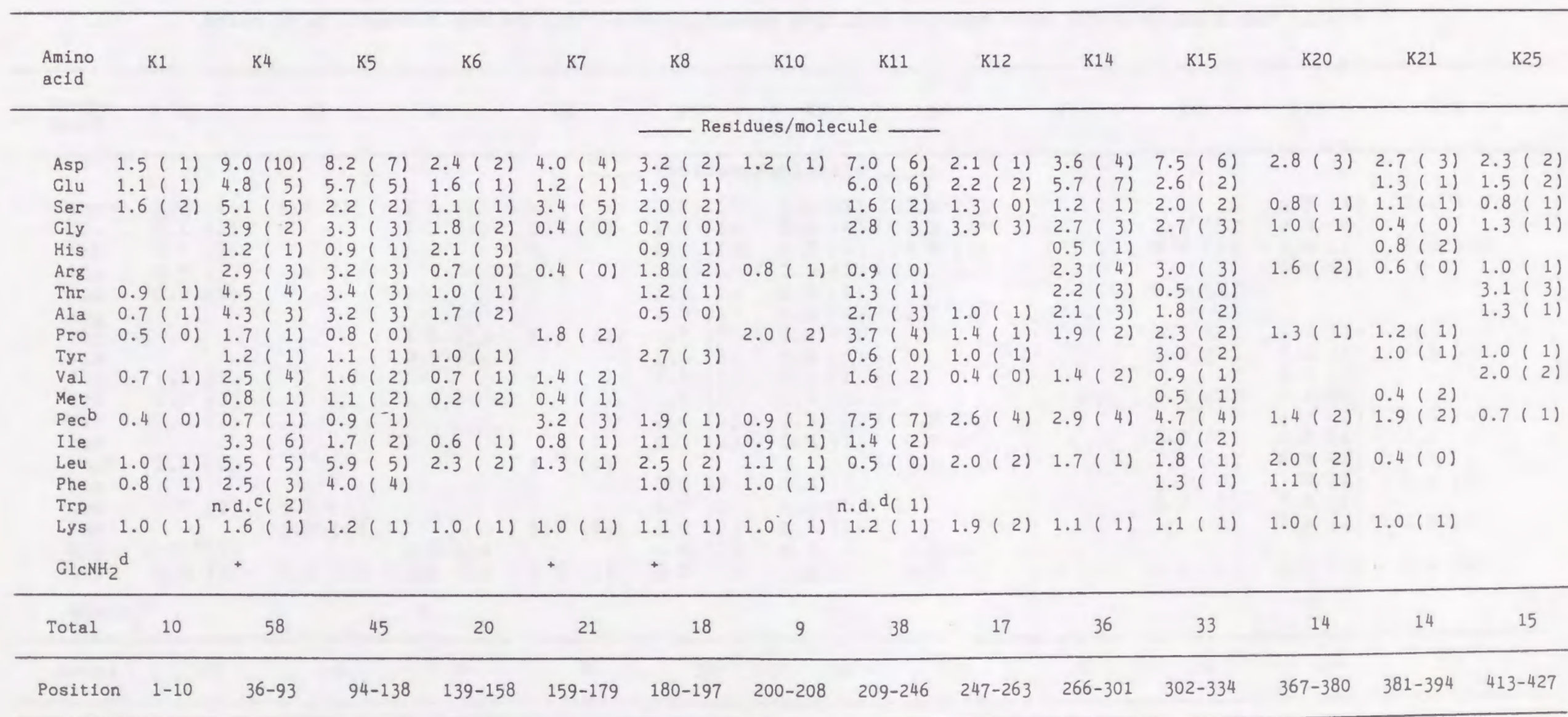

avalues in parentheses are taken from the sequence data.

b-Pyridylethylcysteine.

CNot determined.

dGlucosamine. 
Table IV

Amino acid compositions of lysyl endopeptidase peptides derived from RVV-X-light chain (LC1)a

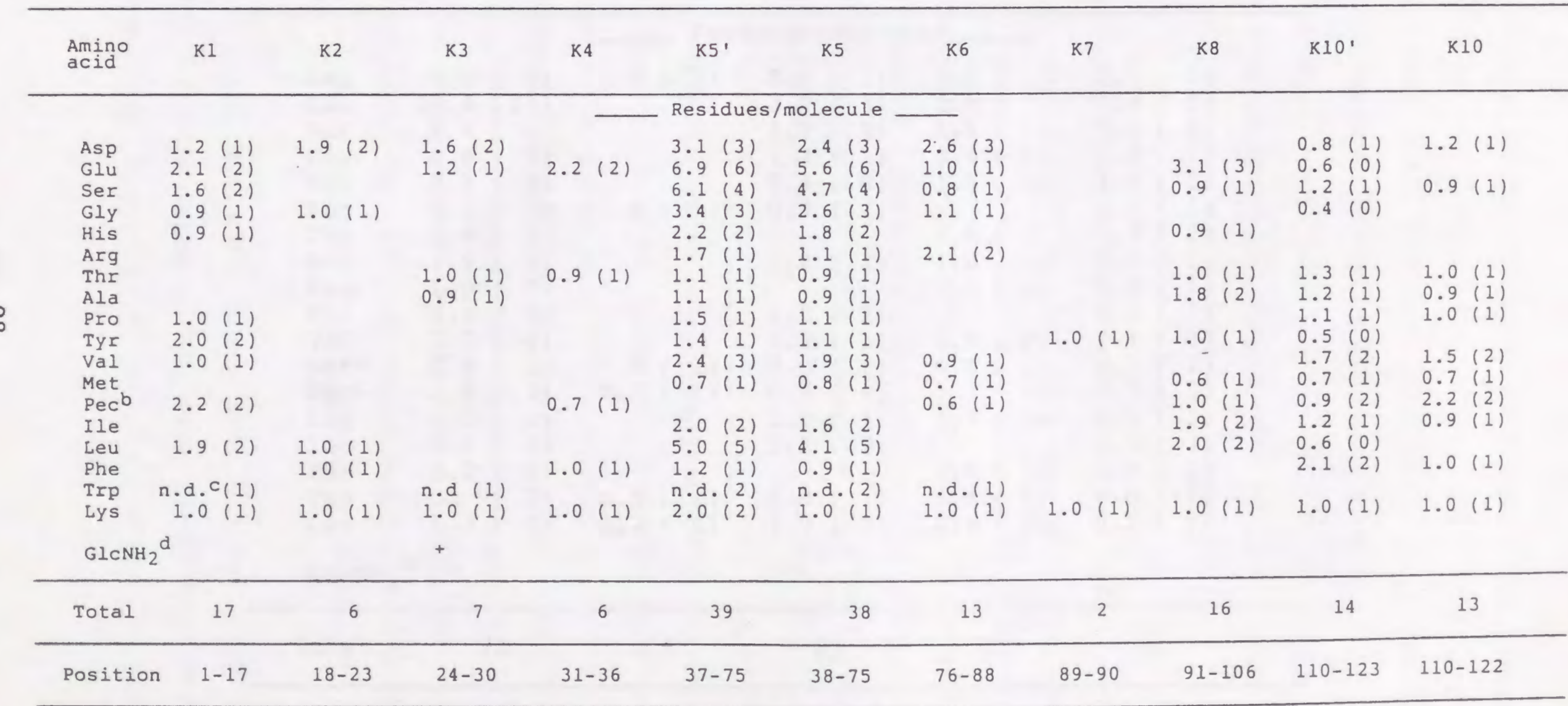

aValues in parentheses are taken from the sequence data.

bs-Pyridyle thylcysteine.

$c \bar{N}$ ot determined.

dGlucosamine. 
Amino acid compositions of cyanogen bromide peptides derived from RVV-X-light chain (LC1)a

\begin{tabular}{|c|c|c|c|c|c|c|}
\hline \multirow{2}{*}{$\begin{array}{l}\text { Amino } \\
\text { acid }\end{array}$} & M1 & \multicolumn{2}{|l|}{$M 2$} & M3 & M4 & \multirow[t]{2}{*}{ M3-4 } \\
\hline & & $\longrightarrow$ & Res & Lues/molec & L & \\
\hline Asp & $8.0(8)$ & 1.11 & 1) & $2.0(2)$ & $1.2(1)$ & $2.7(3)$ \\
\hline Glu & $10.4(11)$ & & & $3.0(3)$ & $2.0(2)$ & $5.4(5)$ \\
\hline Ser & $4.4(6)$ & & & $1.7(2)$ & $1.3(1)$ & $3.5(3)$ \\
\hline Gly & $4.8(5)$ & & & $1.2(1)$ & $0.8(0)$ & $3.9(1)$ \\
\hline His & $2.8(3)$ & & & $0.4(0)$ & $1.0(1)$ & $1.0(1)$ \\
\hline Arg & $1.1(1)$ & 1.01 & 1) & $0.9(1)$ & & $1.5(1)$ \\
\hline Thr & $2.9(3)$ & & & & $1.9(2)$ & $1.7(2)$ \\
\hline Ala & $1.9(2)$ & & & $1.8(2)$ & $1.0(1)$ & $3.3(3)$ \\
\hline Pro & $2.0(2)$ & & & & $1.0(1)$ & $1.0(1)$ \\
\hline Tyr & $2.9(3)$ & & & $2.0(2)$ & & $1.8(2)$ \\
\hline $\mathrm{VaI}$ & $3.2(4)$ & & & $1.0(1)$ & $1.6(2)$ & $2.4(3)$ \\
\hline Metb & 0.6 (1) & 0.91 & 1) & $0.8(1)$ & $0.6(1)$ & $0.2(2)$ \\
\hline $\mathrm{Pec}^{\mathrm{C}}$ & $2.9(3)$ & 0.71 & 1) & $0.7(1)$ & $1.3(2)$ & $1.3(3)$ \\
\hline Ile & $2.0(2)$ & & & $1.0(1)$ & $1.7(2)$ & $2.5(3)$ \\
\hline Leu & $8.1(8)$ & & & $2.2(2)$ & , & $2.7(2)$ \\
\hline Phe & $3.0(3)$ & & & & $1.9(2)$ & $1.9(2)$ \\
\hline $\operatorname{Trp}$ & n.d.di 3$)$ & $\mathrm{n} \cdot \mathrm{d} \cdot($ & 1) & $\mathrm{n} \cdot \mathrm{d} \cdot(1)$ & n.d.( 1) & n.d. ( 2) \\
\hline Lys & $5.3(5)$ & 1.21 & 1) & $1.9(2)$ & $2.4(3)$ & $3.5(5)$ \\
\hline $\mathrm{GlCNH}_{2}{ }^{\mathrm{e}}$ & + & & & & & \\
\hline Total & 73 & 6 & & 22 & 22 & 44 \\
\hline Position & $1-73$ & $74-79$ & & $80-101$ & $102-123$ & $80-123$ \\
\hline
\end{tabular}

avalues in parentheses are taken from the sequence data.

betermined as homoserine.

CS-Pyridylethylcysteine.

d Not determined.

eglucosamine. 
FIG. 1. Separation of RVV-X-heavy (HC) and light chains (LC). S-pyridylethylated RVV-X was subjected to gel filtration HPLC on G3000SW equilibrated with $0.1 \mathrm{M}$ sodium phosphate buffer, $\mathrm{pH} 6.0$, containing $6 \mathrm{M}$ guanidine hydrochloride and $1 \mathrm{mM}$ EDTA. The inset is 12.5 \& SDS-PAGE analysis of the isolated HC and LC. The gels were stained with Coomassie Brilliant Blue R-250.

FIG. 2. Separation of RVV-X-1ight chains, LC1 and LC2, by reversed phase HPLC. The fraction LC shown in Fig. 1 was applied to a column of Vydac 214TP5415. The proteins were eluted at room temperature with a linear gradient of acetonitrile containing $0.1 \%$ TFA at a flow rate of $0.5 \mathrm{ml}$ per min. The inset is $12.5 \%$ SDS-PAGE analysis of the isolated LC1 and LC2. The gels were stained with Coomassie Brilliant Blue R-250.

FIG. 3. The complete amino acid sequence of RVV-X-heavy chain. Amino acid residues are given in single letter code. Residues determined by Edman degradation are given below the summarized sequence. Dashes indicate unidentified residues. $N-$ linked sugar chains are shown by . Pe-heavy chain, Spyridylethylated heavy chain; $M$, CNBr-cleaved peptides; $K$, lysyl endopeptidase-digested peptides; $D$, endoproteinase Asp-N-digested peptides; $T$, tryptic peptides; $C$, chymotryptic peptides.

FIG. 4. Separation of CNBr fragments derived from Pe-heavy chain on a TSK G2000SW column. The same conditions as described for FIG. 1 were used. Fragments M5 and M12, and M3, M4, and M6 were 
separated by reversed phase HPLC on a column of Cosmosil 5C4-300 (data not shown).

FIG. 5. Separation of lysyl endopeptidase-digested peptides derived from Pe-LC1 by reversed-phase HPLC. The digest was applied to a column of $\mu$ Bondasphere C8. A flow rate is $0.2 \mathrm{ml}$ per min. Other conditions are the same as those described for FIG. 2.

FIG. 6. The complete amino acid sequence of RVV-X-light chain (LC1). Amino acid residues are given in single letter code. Residues determined by Edman degradation are given below the summarized sequence. Dashes indicate unidentified residues. Nlinked sugar chain is shown by 0 . Pe-LC1, S-pyridylethylated LC1; K, lysyl endopeptidase-digested peptides; $M$, CNBr-cleaved peptides; $E$, V8 protease-digested peptides.

FIG. 7. Structural comparisons of RVV-X-heavy chain with other proteins. The entire amino acid sequence of RVV-X-heavy chain (427 residues) is similar to that of HR1B (416 residues). HR1B is a high molecular mass hemorrhagic metalloproteinase isolated from the venom of Trimeresurus flavoviridis (16). A, sequence comparison of the amino-terminal regions of RVV-X-heavy chain and HRIB with HR2a, HT-2, hemorrhagic toxin d ( $\mathrm{Ht}-\mathrm{d})$ and $\mathrm{H}_{2}$-proteinase. HR2a (14), HT-2 (17), Ht-d (29) and $\mathrm{H}_{2}$-proteinase (15) are low molecular mass metalloproteinases isolated from the venoms of T. flavoviridis, $C$. ruber ruber, Crotalus atrox, and T. flavoviridis, respectively. The residues shared by hemorrhagic metalloproteinases HR1B, HR2a, HT-2, and Ht-d and not shared by non-hemorrhagic H2-proteinases and RVV-X 
are boxed. Residues conserved in all the proteins are shown at the bottom. The putative zinc ligands and active site are indicated by $\Delta$ and $\Delta$, respectively. $B$, Comparison and alignment of the carboxylterminal regions of RVV-X-heavy chain and HRIB with several disintegrins. Trigramin (30), echistatin (31), bitistatin (32) and barbourin (33) are all viper venom platelet aggregation inhibitors, called disintegrins, isolated from the venoms of Trimeresuras gramineus, Echis carinatus, Bitis arietance, and sistrurus m. barbouri, respectively, Residues conserved in all the proteins are shown at the bottom. Arg-Gly-Asp sequences are boxed.

FIG. 8. Inhibition of platelet aggregation by RVV-X. PRP was incubated with Tris-HCl buffer saline, $\mathrm{pH} 7.5$ or various concentrations of $\mathrm{RVV}-\mathrm{X}$ at $37{ }^{\circ} \mathrm{C}$ for $3 \mathrm{~min}$ before addition of collagen or ADP. Other details are described under "Materials and Methods" .

FIG. 9. Alignment of the amino acid sequence of RVV-X-light chain (LC1) and those of C-type lectin structures. Proteins on upper 4 lanes are originated from snakes, and proteins on lower 6 lanes are originated from mammals. RSL, rattlesnake lectin from the venom of Crotalus atrox (35); PLIA, phospholipase A2 inhibitor A from blood plasma of Trimeresurus flavoviridis (36); IX/XBPA, factor IX/factor $\mathrm{X}$-binding protein A chain from the venom of $T$. flavoviridis (37); MLHR, mouse lymphocyte homing receptor (38); RKCR, rat Kupffer cell receptor (39); RMBPA, rat mannose-binding protein A (40); HFCER, human lymphocyte IgE receptor (41); HTET, human tetranectin (42); HPSAP, human pulmonary surfactant associated protein (43). Residues 
conserved in more than seven proteins are boxed. Dashes are inserted to maximize similarity.

FIG. 10. Inhibition of the RVV-X-catalyzed factor $x$ activation by $I X / X-b p$. Factor $X(3.6 \mu \mathrm{M})$ was activated by $R V V-X$

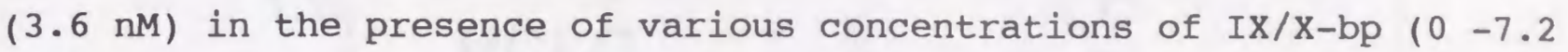
$\mu \mathrm{M})$. The factor Xa activity generated was measured using $\mathrm{N}^{\alpha}$ benzyloxycarbonyl-L-pyroglutamyl-Gly-Arg-4-methylcoumaryl-7-amide as a substrate. The activity in the absence of $I X / X-b p$ was set as 100 \%.

FIG. 11. Gross structures of snake venom metalloproteinases. a, Low molecular mass metalloproteinase including HR2a, HT-2, Ht-d and H2-proteinase $(14,17,29$ and 15). b, Precursor protein of trigramin deduced from its CDNA sequence (49). C, High molecular mass metalloproteinase HR1B (16). d, RVV-X. Identified and potential $N$-linked sugar chains are shown by and $O$. The locations of conserved HEXXH sequence are indicated.

FIG. 12. The amino acid sequence deduced from the previously reported cDNA sequence encoding trigramin. Nucliotide sequence (upper) is taken from Ref. 49 and amino acid sequence is deduced (lower). Putative signal sequence is double-underlined. The potential attachment sites for $N$-linked sugar chains are indicated by - The region that are homologous to the metalloproteinase domain is indicated. The zinc-chelating HEXXH sequence is boxed. The amino acid sequence of mature trigramin is underlined. 


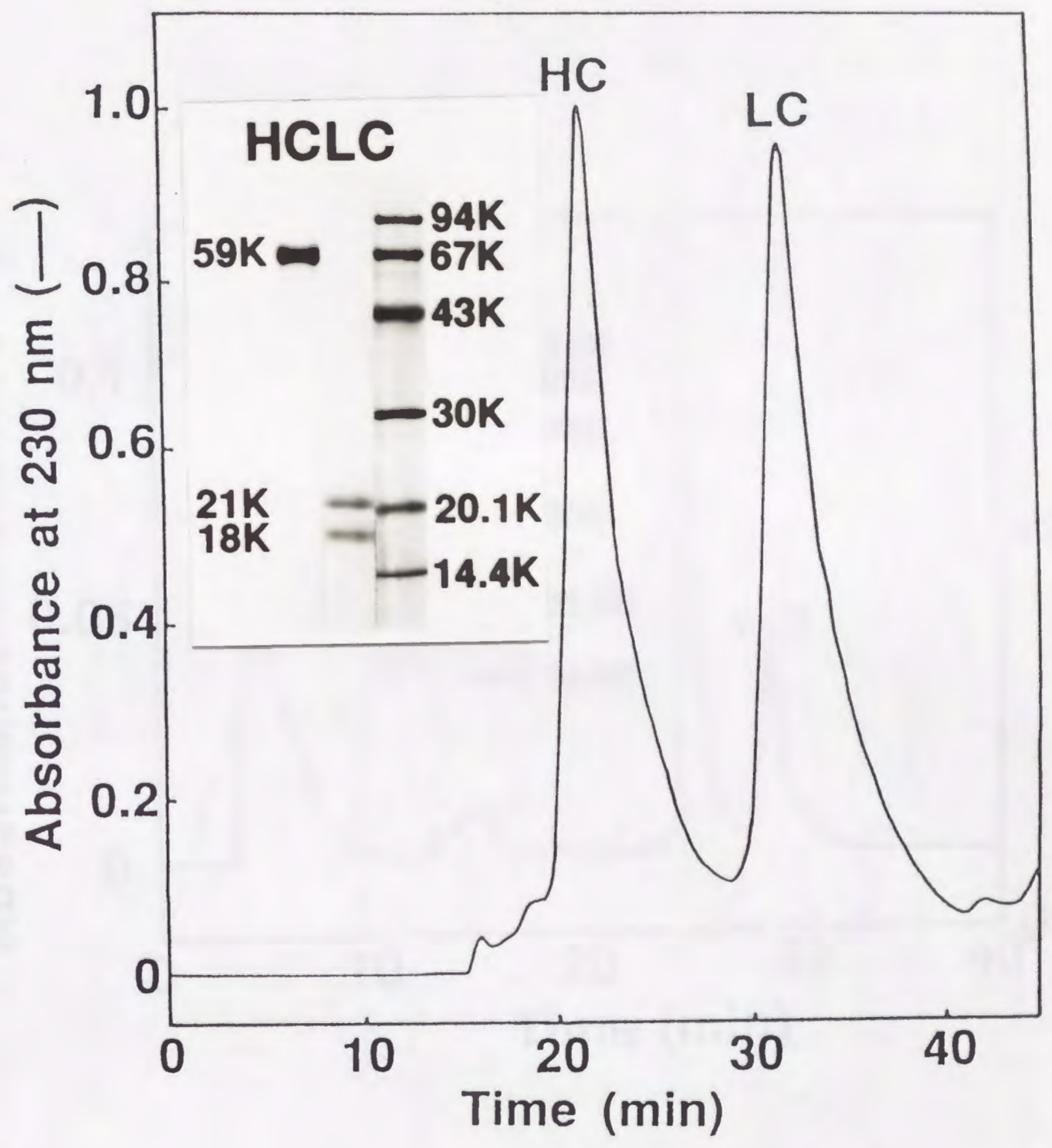

Fig. 1 


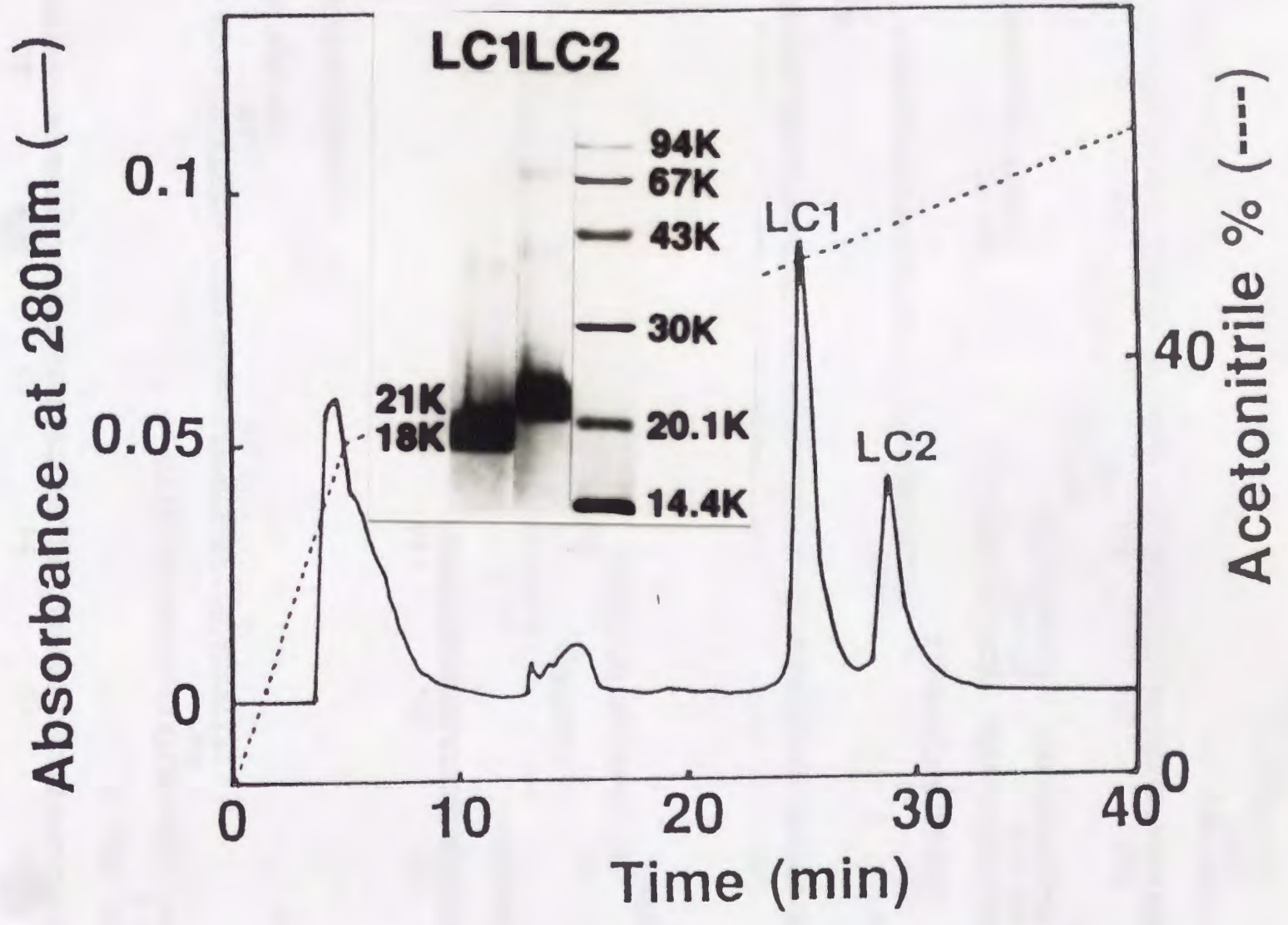

Fig. 2 


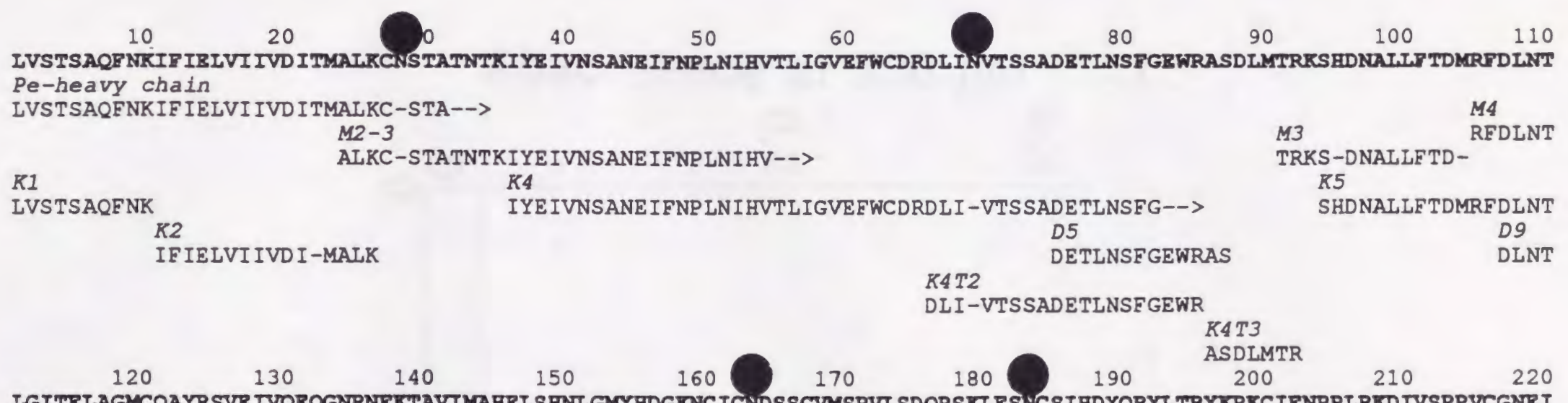

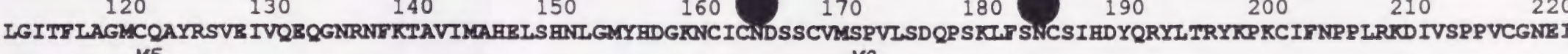

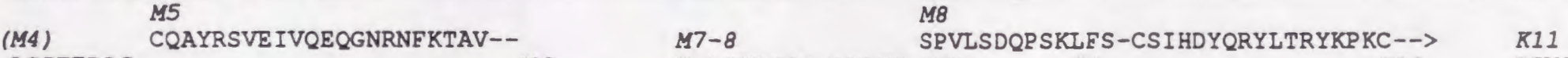
LGITELAGM KE NCIC-DSSCVMSPVLSDQPSK DF-CSIHDYOY

LGITFLAGMCQAYRSVE IVQEQGNRNEK TAVIMAHELSHNLGMYHDGK

DYQRYLTRYKPKCIFNPPLRK

(D9)
LGITE LAGMCQAYRSVEIVQ-->

240

250

260

270

280

290

M7-8C1 $D 14$ KPKCIF 14 IVSPVCGNEI NPPLRKDIVSPRVCGNET

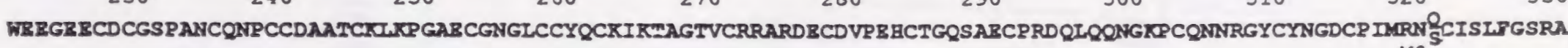
(R11)

WEEGEECDCGSPANCQNPCCDAA---

(D14) D15 R12

TAGTVCRRARDECDVPEHCTGQSAECPRDQLQQNGK $M 9$ D16 KPGAECGNGICCYOCK R15 r RNQCISLFGSRA
PCONNRGYCYNGDCP IMRNOCISLFGSRA (M7-8C2) DAATCKLKPGAECGNGLC-YQCKIKTAGTVCR-AR

WEEGEECDCGSP $-->\quad M 7-8 C 4 \quad M 7-8 C 5$ MC-8C3 KLKPGAECGNGL QCKIKTAGTVC M7-8C6

M7-8C7
SAECPRDQLQQNGKPC D20 DCP IMRNQC ISLEGSRA DCP IMRNSCISLFGSRA $340350360370380^{-R A R D E C D V P E C T G Q}$

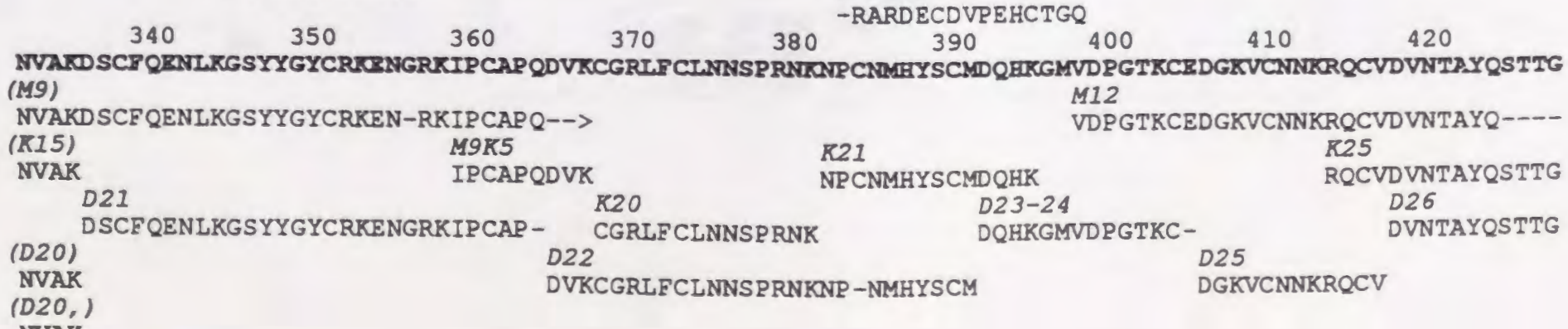
NVAK

Fig. 3 


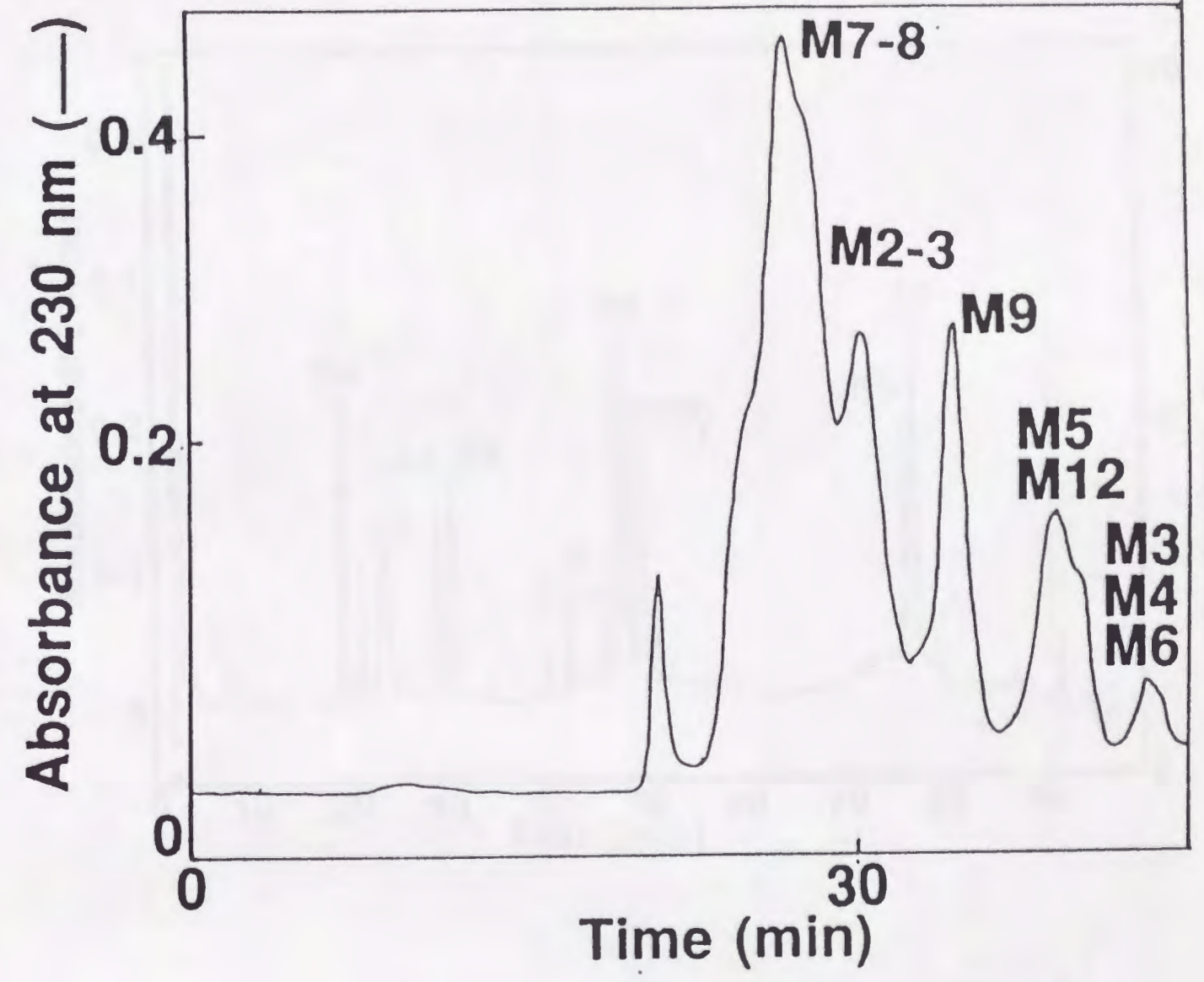

Fig. 4 


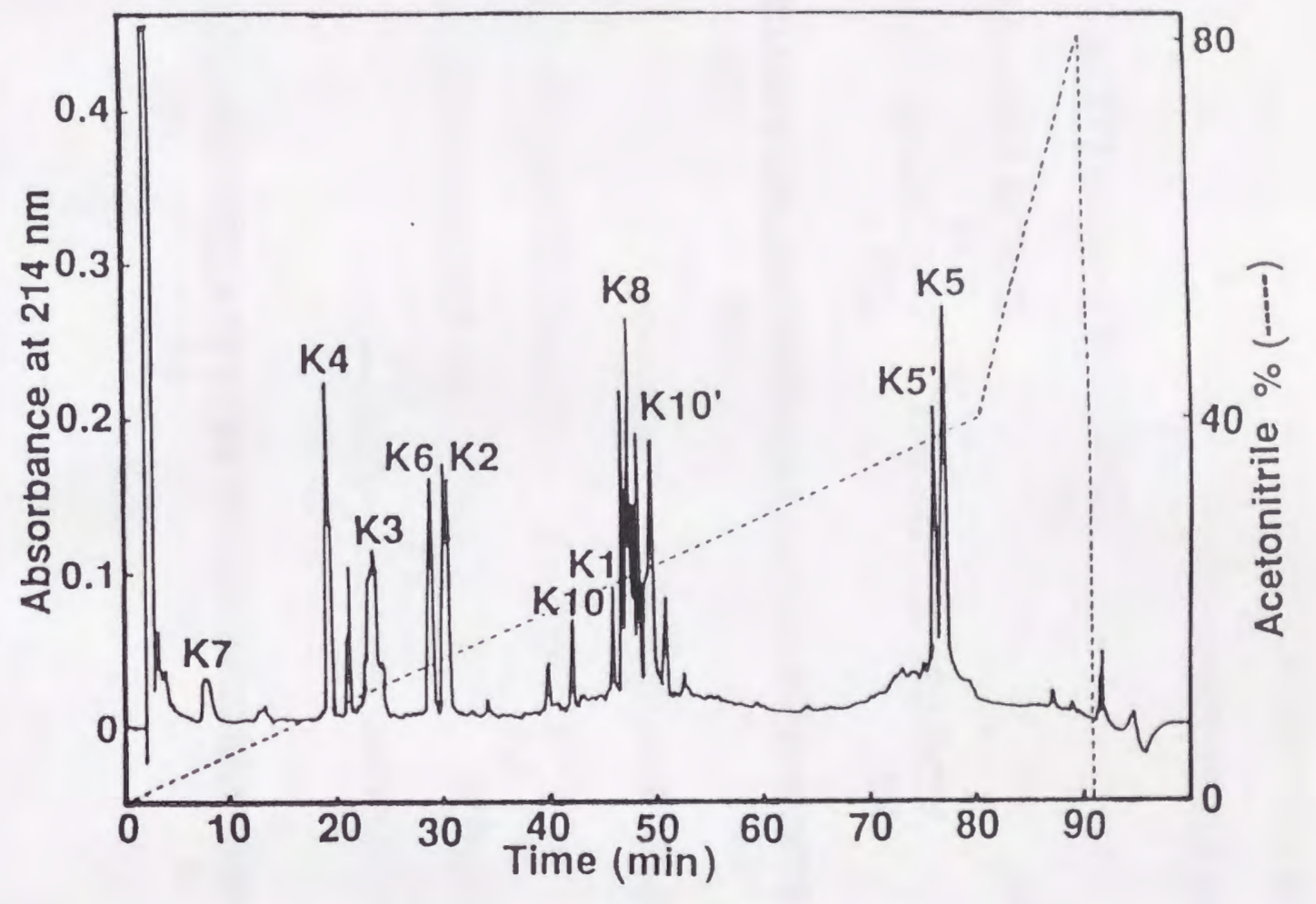

Fig. 5 
RVVXH : LVSTSAOFNKIF IELVI IVDHSMAKKCNSTATNTKIYEIVNSANEIFNP LNIHVTLIGVEFWCDRDLINV HR1B : CEQRFPRRYIKLAIVVDHGIVTKHHGNLKKIRRKW IYQLVNT INNI YRSLNI LVALVYLEIWSKQNKITV <EORFPORY IELAI VVDHGMYTKYSSNFKK IR KRVHQMVNN INEMYRP LNIA IT LS LLDVWSEKDLI TM <ENLPQSY IE LVVVADHRMF MKYNSD LNT IETRVHE IVNF I NEFYRS LN IRVSLTD LE IWSDQDF ITV $\angle E O N L P O R Y I E L V V V A D H R V F M K Y N S D L N T$ IRTRVHE IVNF I NGF YRS LNI HVSLTDLE IWSNEDOINI $\angle E R F P Q R Y I E L A$ IVVDHGMYKKYNQNSDKI KVRVHQMVNH I NEMYRP LNIA I S LNRLQ IWSKKDLITV

Consensus :

RVVXH

HR1B

HR2a

HT -2

Ht $-\mathrm{d}$

$\mathrm{H} 2$

Consensus

RVVXH

HR1B

HR2a

$\mathrm{HT}-2$

$\mathrm{H} t-\mathrm{d}$

H2

Consensus

\section{0}

90

100

110

120

130

140

TS SADETLNSFGEWRASDLMTRKSHDNALLFTDMRFD LNTLGI TFLAGMCOAYRSVE IVOEOGNRNFKTA CSASNVT LDLFGDWRESVLLKQRSHDCA ILLTT I PFD GPT I GKAYTASMCDPKRSVGIVQDYSP INLVVA QAVAPTTARLF GDWRETVLLKOKDHDHA LLTDI NFT TNT I GWAYMGGMCNAKNSVGIVKDHS SNVE'MVA DS SAKNTLHSF GEWRKSVLLNRKDHDNA ILLTA IVLDDYTLGLAY LNSMCHPRNSVGL IQDHSP INLLMG dSASSDT LNAFAEWRETDLLNRKS HDNACLLT AIELDEETLGLAP LGTMCDPKLS IGIVQDHSP INLLMG KSASNVTLESFGNWRETVLLKQQNNDCAHLLTATNLNDNTI GLAYKKGMCNPKLSVGLVQDYSPNVEMVA

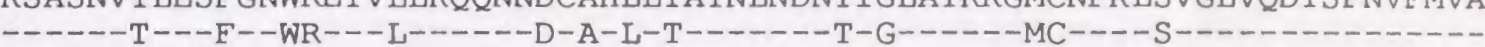
150 160 170 180 190 200

VIMAHE L SHNLGMYHDGKN-CI CNDS SCVMSPVLSDOP SKLF SNCS IHDYORYLTRYKPKCIFNPPLRKD VIMTHEMGHNLG IPHDG-NSCTCGGFPCIMSPMI SDP P SELF SNCSKAYYYTF LTDHKPQCI LNAP SKTD VTMTHE I GHNLGMEHDDKDKCKCEA--C IMSAV I SDKP SKLF SDCSKDYYQTF LTNSKPQCI INAP VTMAHELGHNLGMEHDGKD-CLRGASLCIMRP GLTP GRSYEF SDASMPYY YKF LDQYKPQCI LNKP VTMAHELGHNLGMEHDGKD-CLRGAS LCIMRP GLTKGRSYEF SDDSMHYYERF LKQYKPQCI LNKP VTMTHELGHNLGMEHDDKDKCKCEA--CIMSDVISDKP SKLF SDCSKNDYQTFLTKYNPQCILNAP $\mathrm{V}-\mathrm{M}-\mathrm{HE}--\mathrm{HNLG}--\mathrm{HD}-\cdots-\mathrm{C}-\cdots--\mathrm{C}-\mathrm{M}-\cdots---\mathrm{S}--\mathrm{FS}--\mathrm{S}-\cdots \mathrm{Y}---\mathrm{L}---\mathrm{P}-\mathrm{CI}-\mathrm{N}-\mathrm{P}-\cdots-\mathrm{D}$ $\triangle \triangle$

\section{B.}

RVVXH

HR1B

Trigramin

Echistatin :

Bitistatin :

Barbourin

Consensus

RVVXH

HR1B

Trigramin

Echistatin :

Bitistatin:

Barbourin

Consensus

RVVXH

HRIB

Consensus

RVVXH

HR1B

Consensus
220

230

240

250

260

270

IVSPPVCGNE IWEEGEECDCGSP ANCONPCCDAATCKLKP GAECGNGLCCYOCKIKTAGTVCRRARDEC IVSPPVCGNELLEAGEECDCGSPENCQYOCCDAASCKLHSWVKCESGECCDQCRFRTAGTECRAAESEC EAGEDCDCGSPAN--P-CCDAATCKLIPGAQCGEGLCCDQCSFIEEGTVCRI A RGDECESGPCCRNCKFLKEGTICKRARGC-

SPPVCGNKI LEQGEDCDCGSPANCQDQCCNAATCKLTPGSQCNHGECCDQCKFKKARTVCRIARGLEAGEECDCGSPEN--P-CCDAATCKLRPGAOCADGLCCDOCRFMKKGTVCRVAKGD-

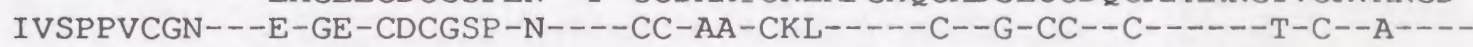
280
290
300
310
320
330
340

DVPEHCTGQSAECPRDQLQQNGKPCQNNRGYCYNGDCP IMRNQC IS LEGSRANVAKDSCEOENLKGSYY DIPESCTGQSADCP TDRFHRNGQPCLYNHGYCYNGKCP IMF YQCYF LFGSNATVAEDDCENNNKKGDKY DLDDYCNGRSAGCPRNPEH

DMDDYCNGKTCDCPRNPHKGPAT

WNDDYCTGKSSNCPWNH

WNDDTCTGQSADCPRNGLYG

$---C-$ G-- - CP-- - - - PC- -N-GYCYNG-CP IM--QC--LFGS-A-VA-D-CE--N-KG--Y
350
360
370
380
390
400
410

GYCRKENGRK IP CAPQDVKCGRLFCLNNSPRNKNP CNMHYSCMDQHKGMVDP GTKCEDGKVCNNKROCV FYCRKENEKY I PCAOEDVKCGRLFCDNKK----YPCHYNYS-EDLDFGMVDHGTKCADGKVCSN-ROCV -YCRKEN---IPCA--DVKCGRLFC-N------PC---YS--D---GMVD-GTKC-DGKVC-N-RQCV

420

DVNTAYOSTTG

DVNEAYKS

DVN-AY-S-.. 
1 min

collagen

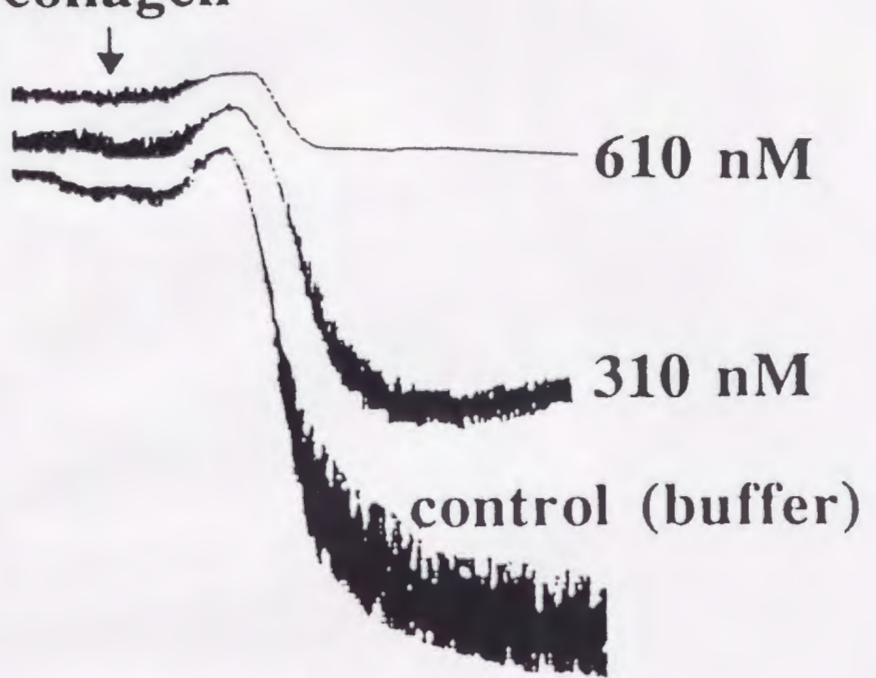

$1 \mathrm{~min}$

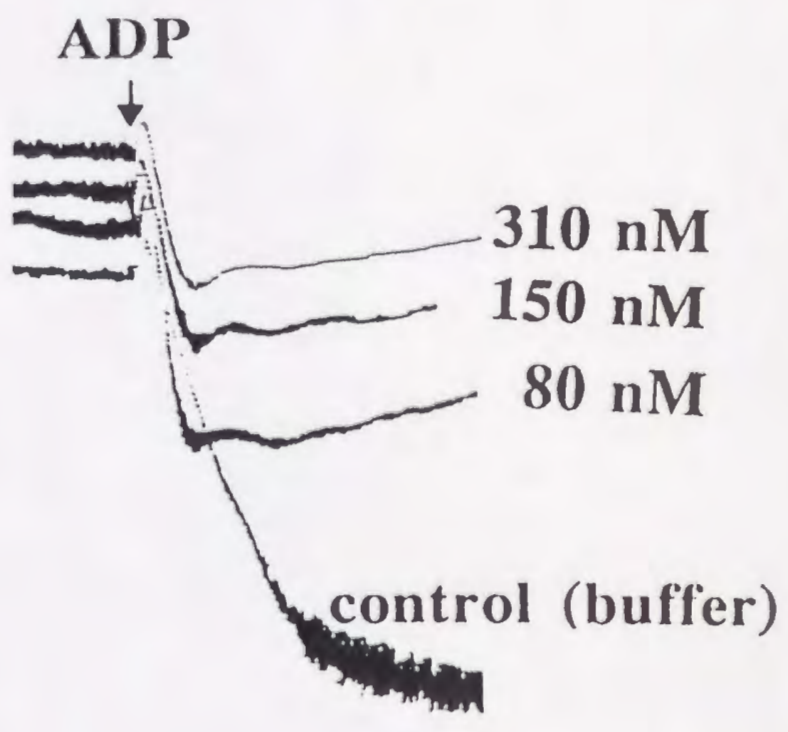

Fig. 8 


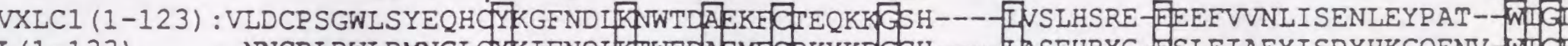

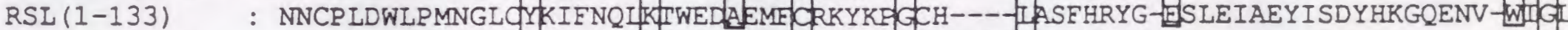
PLIA (33-143) :TVNNARSEGSGSERIYVSNKE IKTFEPLKEICEEA-CGHIPSPQLENQNKAF-ASV-LERHNKAAYL------VIGP

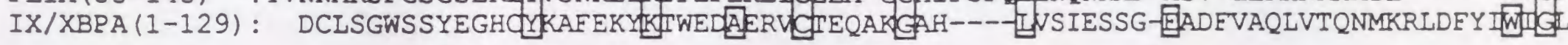

MLHR (1-119) : WTYKYSEKPMNWENARKFCKEN-YTD-D-D-UVAIENKR-EIEYIENTIPKSPYYY-D-WTGI

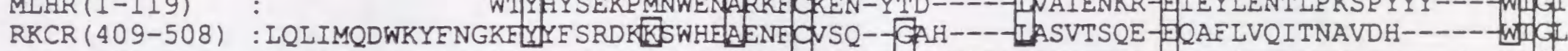

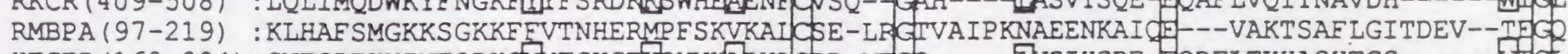

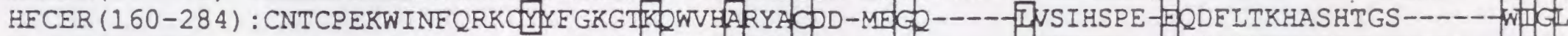
HTET (47-178) :OTVCLKGTKVHMK-CFLAFTQTKTFEEASEDCIS-RGGT-D--DGTPOTGS-ENDALYEYLROSVGNEAEI-WLGT HPSAP (124-248): ALSLQGSIMTVGEKVF SSNGQSITFDAIQEACAR-AGGRIAV--PRNPEEN--EA--IASFVKKYNTYAYVGLTEGP

80

90

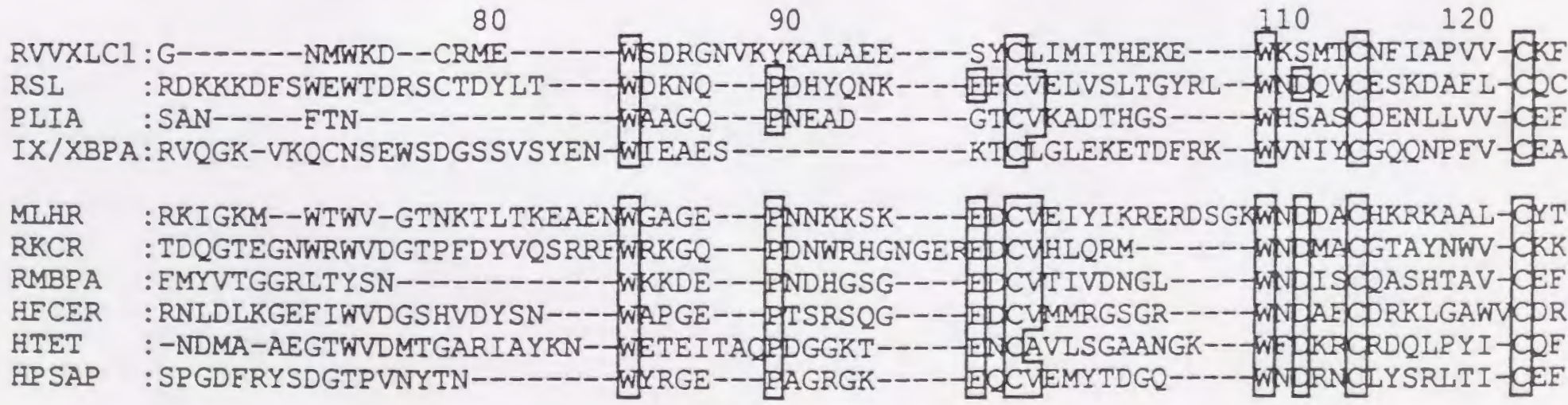

Fig. 9 


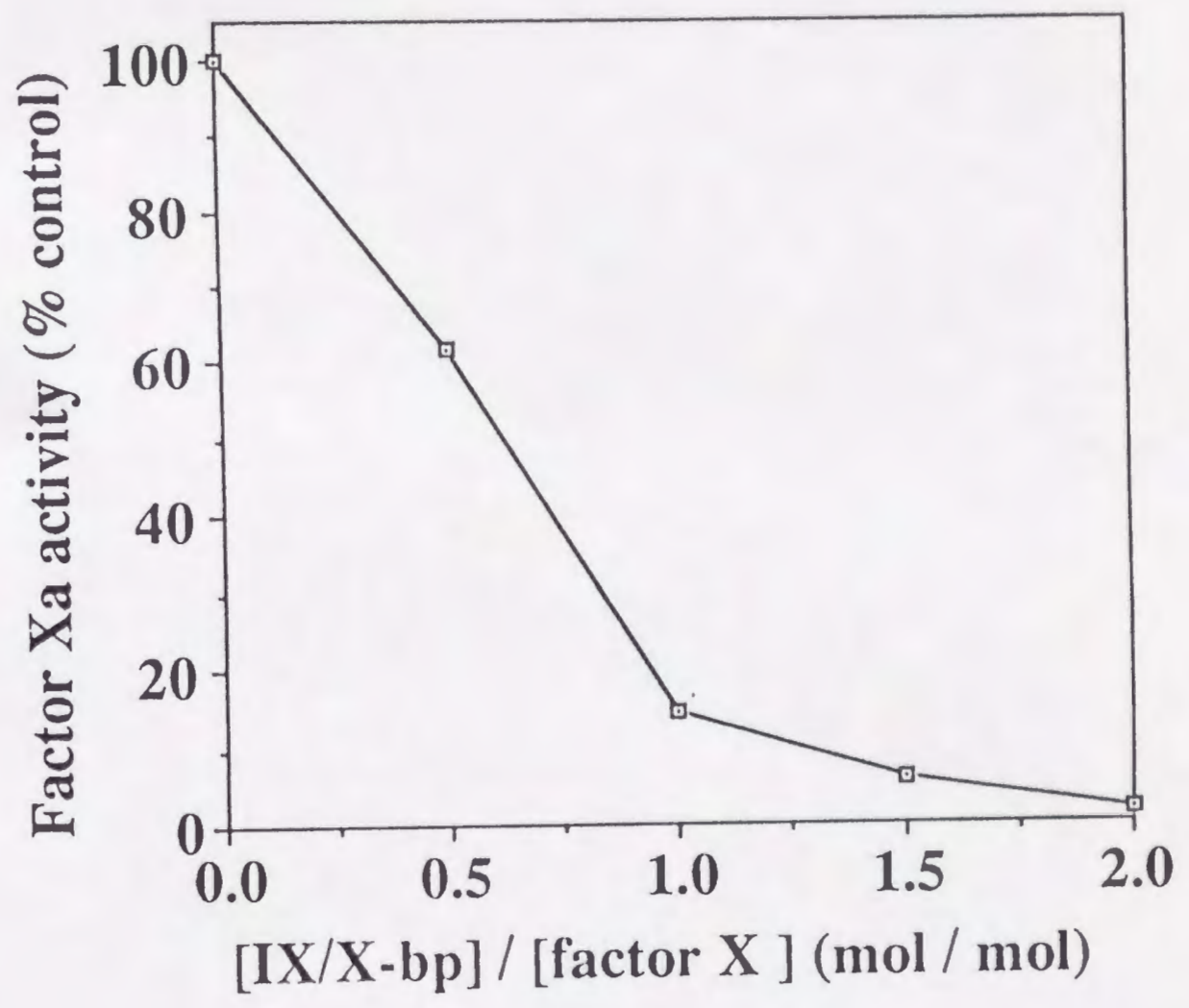

Fig. 10 
a

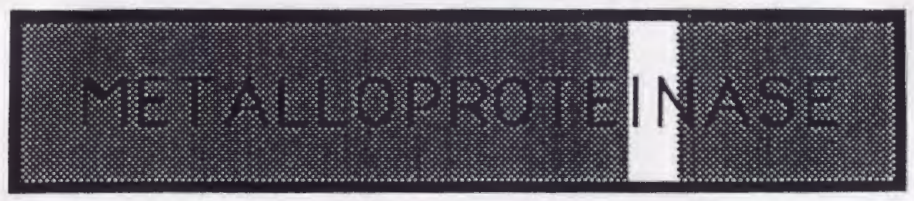

b

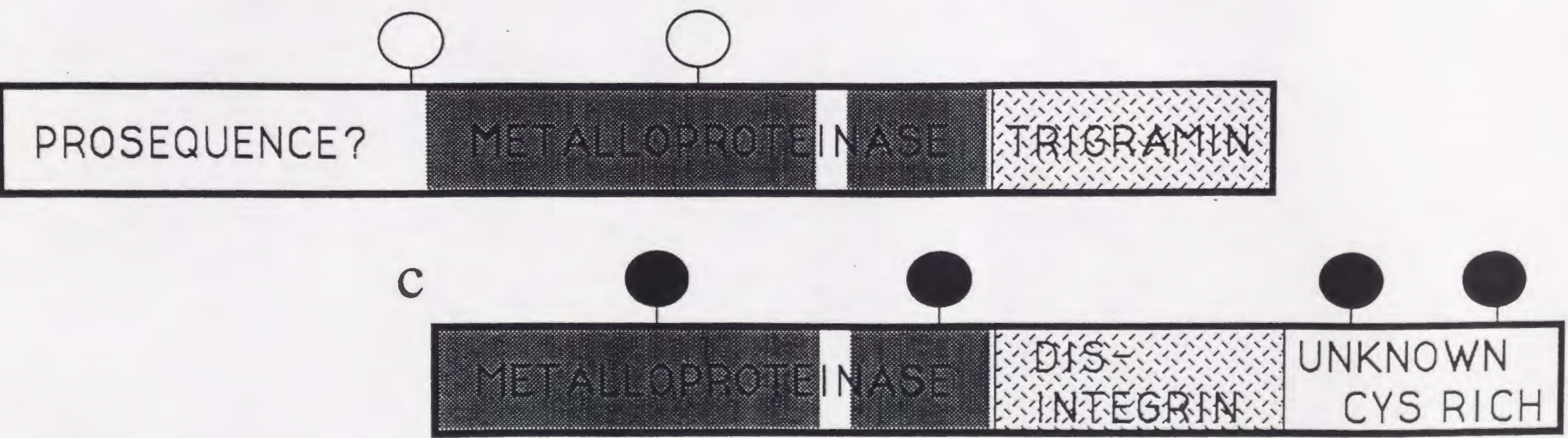

d
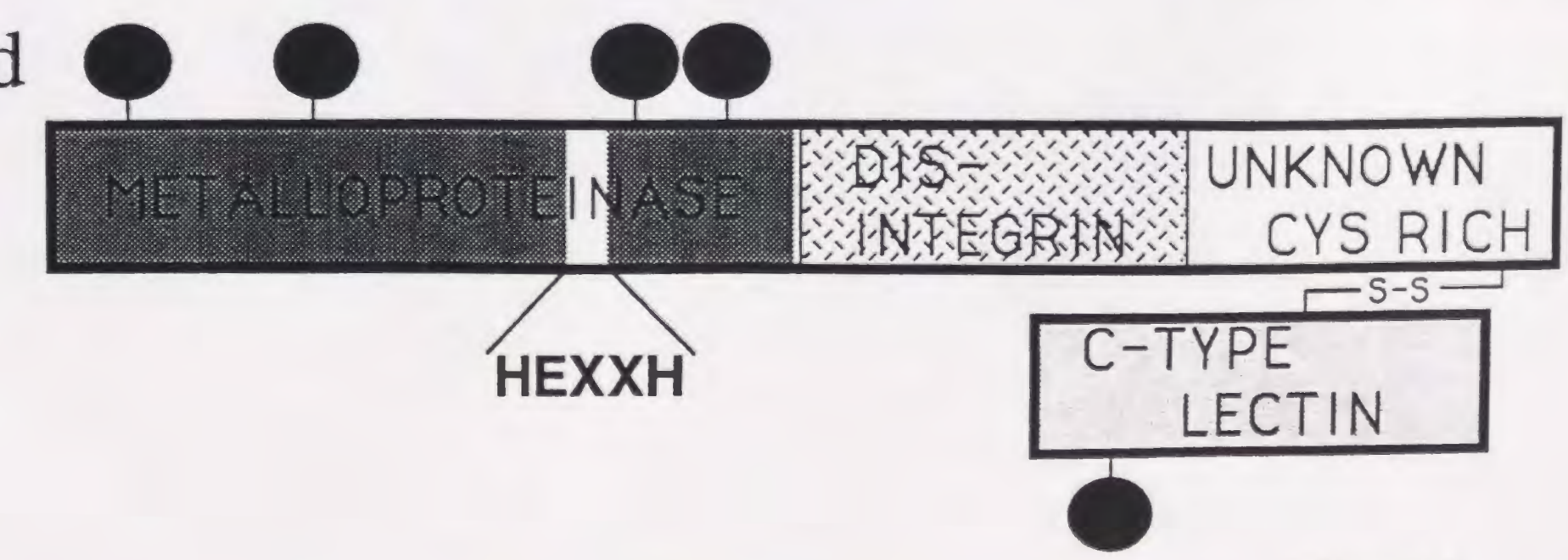

Fig. 11 
CGCTCAGGTTGGCTTGAAAGCAGGAAGAGATTGTCTGTCTTCCAGCCAAATCCAGTCTCCAAAATGATCCAAGTTCTTTTGATAACCATATGCTTAGCAGTTTTTCCTTATCAAGGGAGC

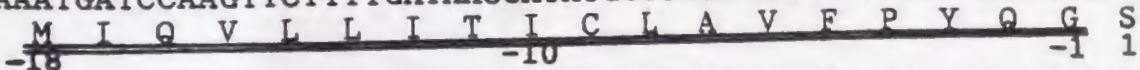

TCCATAATCCTGGAATCTGGGAATCTTAATGATTATGAAGTAGTGTATCCAGAAAAAGTCACTGCATTGCCCAAAGGAGCTGTTCAGCAAAAGTATGAAGACGCCATGCAATATGAATTT

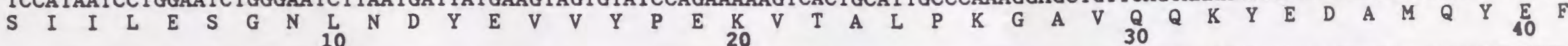
AAGGTGAATGGAGAGCCAGTGGTCCTTCACCTGGAAAAAAATAAAGGACTTTTTTCAGAAGATTACAGCGAGATTCATTATTCCCCTGATGGCAGAGAAATTACAGCATACCCTTCGGTT

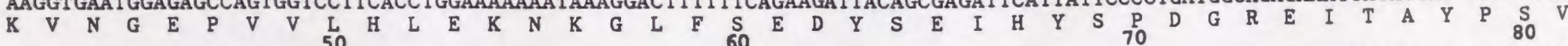
GAGGATCACTGCTATTATCATGGACGCATCGAGAATGATGCTGACTCAACTGCAAGCATCAGTGCATGTGATGGTTTGAAAGGACATTTCAAGCTTCAAGGGGAGATGTACCTTATTGAA

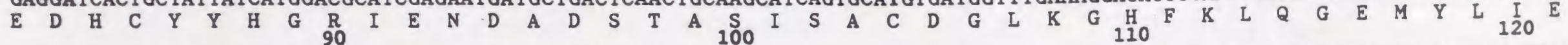
CCCTTGGAGCTTTCCGACAGTGAAGCCCATGCAGTCTTCAAATATGAAAATGTAGAAAAAGAGGACGAGCCCCCCAAAATGTGTGGAGTAACCCAGAATTGGGAATCATATGAGTCCACC

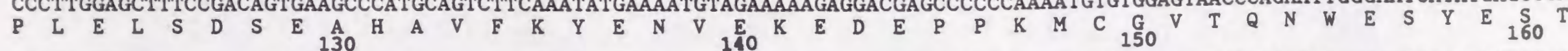
AAAAAGGCCTCTCAGTTAAATGTTACTCCTGAACAACAAAGATTCCCCCAAAGATACATTAAGCTTGGTATATTTGTGGACCACGGAATGTACACAAAATACAGTGGCAATTCTGAAAGG

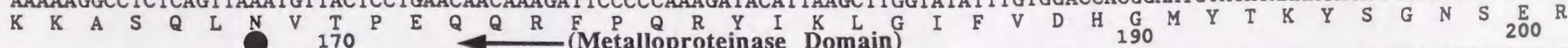

- ATAACAAAAGGGTACATCAAATGATCAACAATATAAATATGATGTGCAGAGCTCTGAATATTGTTACAACACTGAGTGTCCTAGAAATTTGGTCCGAAAAAGATTTGATTACGGTGCAG

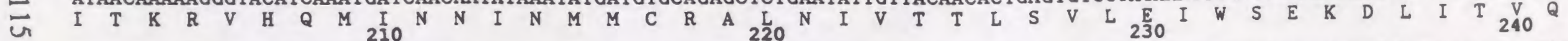
GCATCAGCGCCTACTACTTTGACCTTATTTGGAGCCTGGAGAGAGACAGTCTTGCTGATCGCACCAGTCATGATCATGCTCAGTTACTCACGGCCACTATCTTCAATGGAAACGTTATA

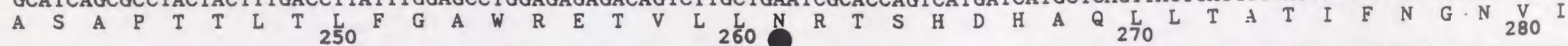
GGAAGGGCTCCCGTGGGCGGTATGTGTGACCCGAAGCGTTCTGTAGCAATTGTTCGGGATCATAACGCAATAGTTTTTGTGGTTGCAGTTACAATGACCCATGAGATGGGTCATAATCTG

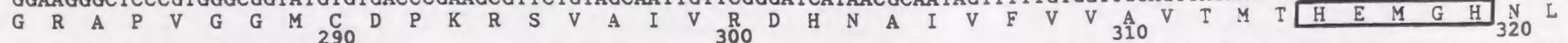
GGCATGCATCATGATGAAGATAAATGTAATTGTAACACATGCATTATGTCTAAAGTGTTAAGCCGCCAACCTTCCAAATATTTCAGCGAATGTAGTAAGGATTATTATCAGACATTTCTI

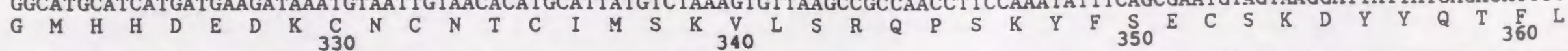
ACTAATCATAACCCACAATGCATTCTCAATGCACCCTTGAGAACAGATACTGTTTCAACTCCAGTTTCTGGAAATGAACTTTTGGAGGCGGGAGAAGATTGTGACTGTGGCTCTCCTGCA $T$ N H N P Q C I L N A P L R T D T V S T T P V S G AATCCGTGCTGCGATGCTGCAACCTGTAAACTGATACCCGGGGCGCAGTGTGGAGAAGGACTGTGTTGTGACCAGTGCAGCTTTATAGAAGAAGGACAGTATGCCGGATAGCAAGGGGT

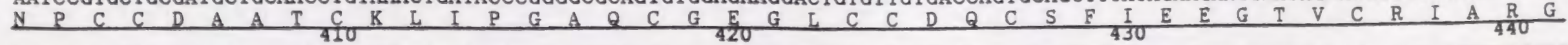
GATGACCTGGATGATTACTGCAATGGCAGATCTGCTGGCTGTCCCAGAAATCCCTTCCATGCCTAACCAACAATGGAGCTGGAATGGTCTGCAACAGCAACAGGCAGTGT GCTGATGTGA D D I D D Y C N G R S A G C P R N P F H A

ATACAGCCTACTAATCAACCTCTGGCTTCTCTCAGATTTGATTTTGGAGATCCTCCTTCCAGAAGGTTTGGCTTCCCTGTAGTCCAAAGAGACCCATCTGCCTGCATCCTACTAGTAAAT CACTCTTAGCTTTCATATGGAATCTAAATTCTGCAATATTTCTTCTCCATATTTAATCTGTTTACCTCTTGCTGTAATCAAGCCTTTTTCCCACCACAAAGCTCCATGGATATGTACAAG ACCAAGGGCTTATTTGCTGTCAAGAAAAAAAATAGCCATTTTACCGTTTCCCAATTCCAGAGCACATTTAATGCAACAGGTTCTGCCTTTAGAGCTGGTGTATTCAAAGTCAATGTTTC CTCTCCCTCTCCCAAAATTCATGCTGGCTTTCCAAGATGTAATTGCTTCCATCAATAAACTCACTATTCTCATTCAAAAAAAAAAAAAAAAAAAAA 


\section{CONCLUSION}

Previously, we have shown that a low molecular weight hemorrhagic protein (HR2a, 202 amino acid residues) isolated from venom of Trimeresurus flavoviridis is a member of new subfamily of metalloproteinases. In the present study, the complete amino acid sequences of a high molecular mass hemorrhagic protein HR1B isolated from the same venom and RVV-X, the blood coagulation factor $\mathrm{X}$ activating enzyme, isolated from Russell's viper venom have been determined. Moreover, the structure-function relationship of these enzymes have been studied. HR1B, is a mosaic protein composed of 416 amino acid residues containing four Asn-linked oligosaccharide chains. The amino-terminal half (residues 1-203) of HRIB contains a metalloproteinase domain, the sequence of which is $62 \%$ identical with that of HR2a and $52 \%$ identical with that of Ht-d isolated from the Crotalus atrox venom. The most interesting finding is that the middle region (residues 204-300) of HR1B shows a striking similarity to disintegrins, Arg-Gly-Asp-containing platelet aggregation inhibitors, recently found in several viper venoms. This result suggests that the middle portion of HR1B may be important to stimulate synergistically hemorrhagic activity with the aminoterminal metalloproteinase domain. Interestingly, however, this region of HR1B does not contain the Arg-Gly-Asp-sequence which is known to be a putative binding site in the disintegrin to the platelet fibrinogen receptor, glycoprotein IIb/IIIa complex.

On the other hand, RVV-X (Mr 79,000) consisted of a disulfide-bonded two-chain glycoprotein with a heavy chain of $\mathrm{Mr}$ 59,000 and a light chain of heterogeneous $\operatorname{Mr} 18,000$ (LC1) or 
21,000 (LC2). The heavy chain had 427 residues containing four asparagine-linked oligosaccharides, and its entire sequence was similar to that of HR1B. The heavy chain contained three distinct domains, metalloproteinase, disintegrin (platelet aggregation inhibitor)-like and unknown cysteine-rich domains. The light chain LC1 consisted of 123 amino acid residues containing one asparagine-linked oligosaccharide and showed a sequence homology similar to those found in the so-called C-type ( $\mathrm{Ca}^{2+}$-dependent) lectin. Therefore, RVV-X is a novel metalloproteinase containing a mosaic structure with disintegrin-like, cysteine-rich, and ctype lectin-like domains. RVV-X potently inhibited collagen- and ADP-stimulated platelet aggregations, probably via its disintegrin-like domain, although this domain does not contain the Arg-Gly-Asp sequence which is conserved in various venom disintegrins and which is thought to be one of the interaction sites for platelet integrins. Our findings also indicate that snake venom factor IX/factor $\mathrm{X}$-binding protein with a C-type lectin structure inhibits the RVV-X-catalyzed factor $\mathrm{X}$ activation, hence, the light chain of RVV-X probably participates in recognizing some portion of the zymogen factor $x$.

These findings indicate the structural and evolutional relationship among these proteins, although each protein has diversed functional activity. These mosaic structures are also analogous to those of mammalian blood clotting serine proteinases and matrix metalloproteinases. While a common domain with apparently distinct functions can be defined among these proteinases, additional domains unique to individual proteinases may have specific functions. 


\section{ACKNOWLEDGMENTS}

The study described in this thesis has been carried out under the guidance of Professor Sadaaki Iwanaga of Kyushu University. This thesis could not have been written without the cooperation, encouragement, and contributions of many people. I am grateful to Professor Sadaaki Iwanaga for his considerate guidance with magnanimity throughout this work. I am also grateful to Drs. Toshiyuki Miyata, Tamotsu Omori-Satoh, Takashi Morita, Yukari Saisaka, and Masaaki Moroi for helpful advise, discussion and continuous encouragement and to Miss Kyoko Oda, and Messrs. Sohichiro Kawada and Shinji Nishida for their cooperation. I am indebted to Mrs. Satsuki Hori-Kajiyama, and Misses Yuko Nishina and Sawako Oyama for amino acid and sequence analyses, to Mrs. Nobuko Ueno-Hashimoto and Itsuko Edamitsu for secretarial assistance, and Miss Mariko ohara for reading the manuscripts. I wish to thank all the members of the laboratory of Professor Sadaaki Iwanaga. 


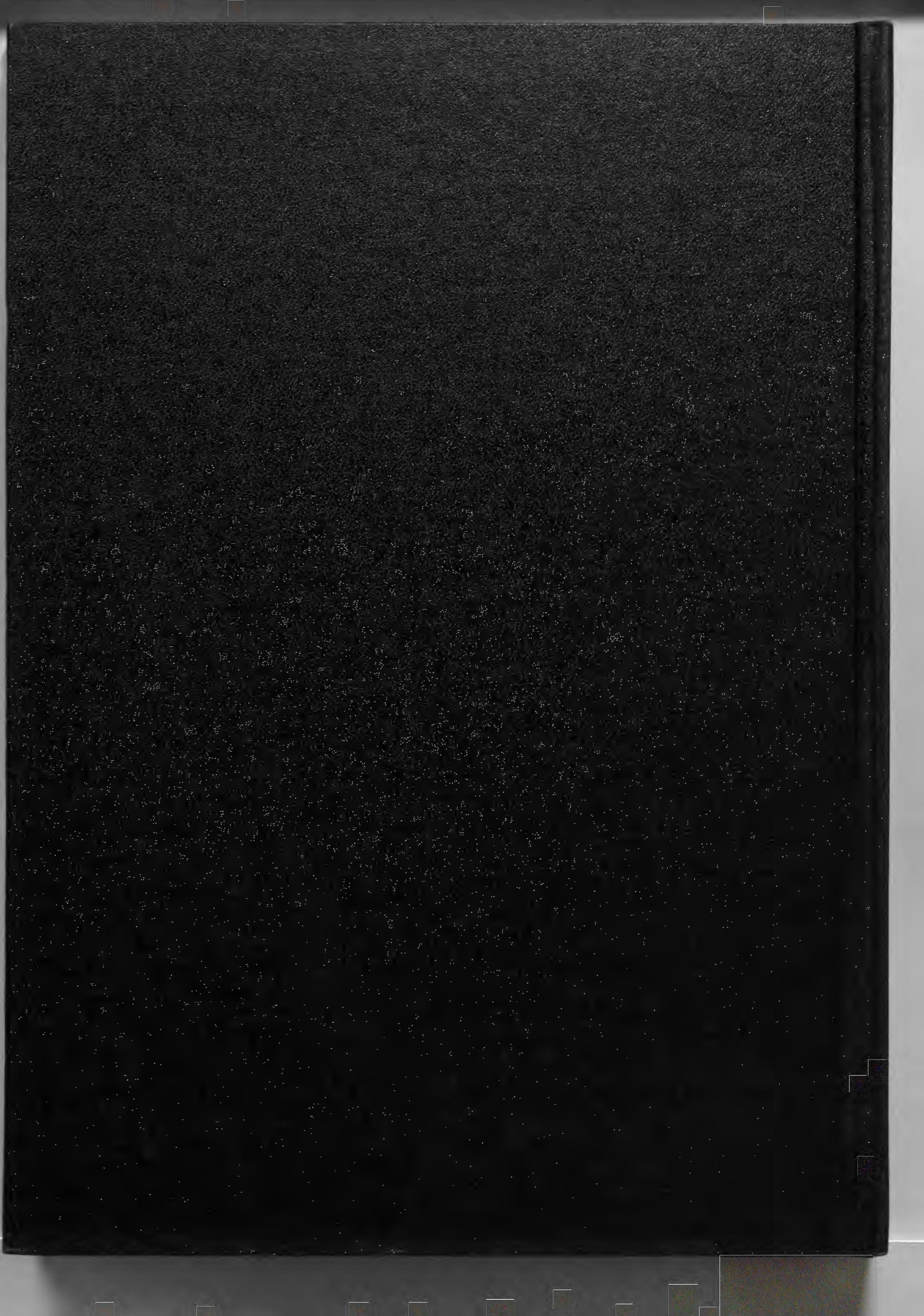




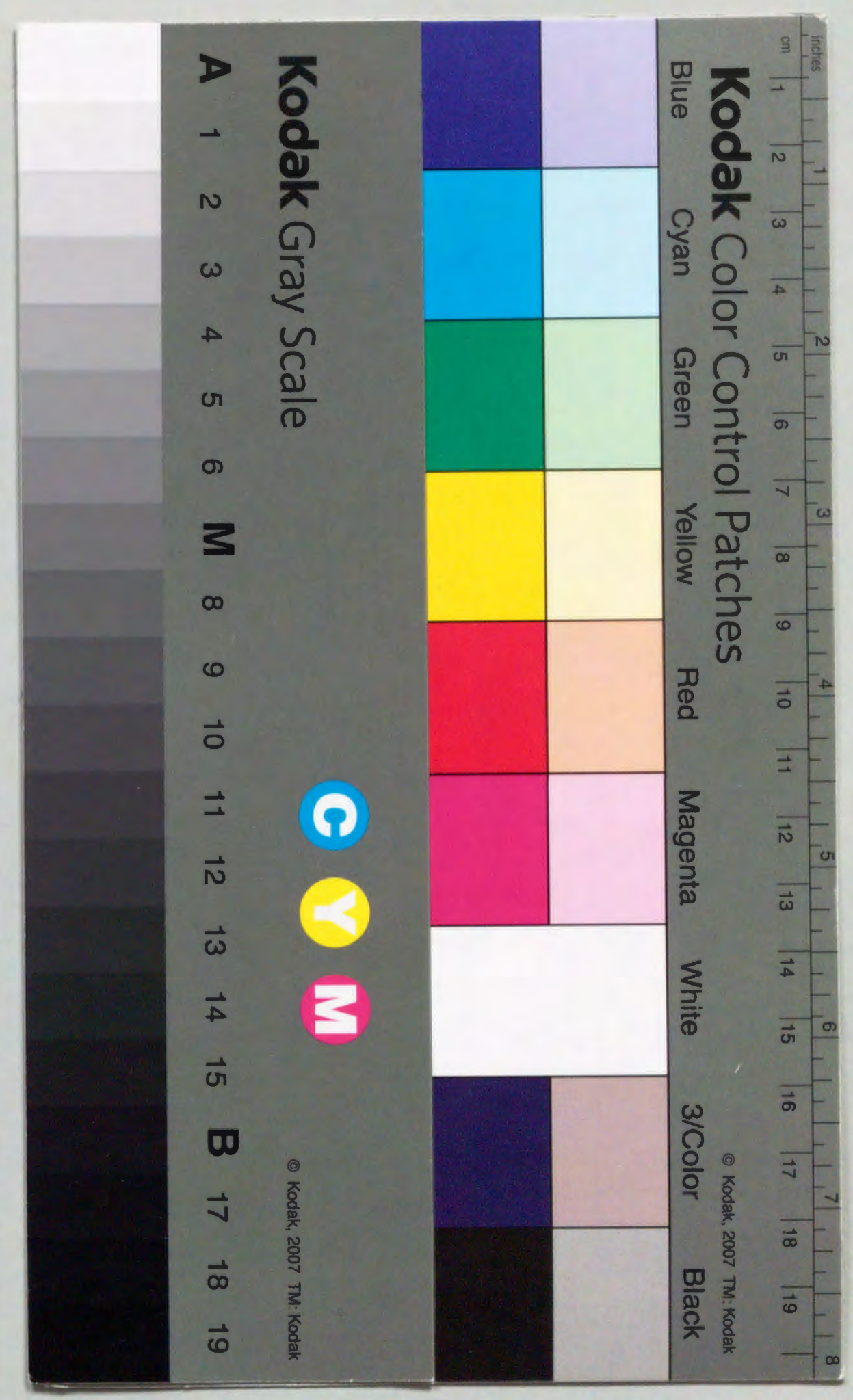

